\title{
Transport in Proton Conductors for Fuel-Cell Applications: Simulations, Elementary Reactions, and Phenomenology
}

\author{
Klaus-Dieter Kreuer, ${ }^{\star \ddagger}$ Stephen J. Paddison,§ Eckhard Spohr,\# and Michael Schuster ${ }^{\ddagger}$ \\ Max-Planck-Institut für Festkörperforschung, Heisenbergstr.1, D-70569 Stuttgart, Germany, Department of Chemistry, \\ University of Alabama in Huntsville, Huntsville, Alabama 35899, and Forschungszentrum Jülich, D-52425 Jülich, Germany
}

Received March 4, 2004

\section{Contents}

1. Introduction

4637

2. Theoretical Methodologies and Simulation Tools

2.1. Ab Initio Quantum Chemistry

2.2. Molecular Dynamics

4640

4641

221. Classical Molecular Dynamics and Monte Carlo Simulations

2.2.2. Empirical Valence Bond Models

4643

2.2.3. Ab Initio Molecular Dynamics (AIMD)

2.3. Poisson-Boltzmann Theory

2.4. Nonequilibrium Statistical Mechanical Ion Transport Modeling

2.5. Dielectric Saturation

3. Transport Mechanisms

3.1. Proton Conduction Mechanisms

3.1.1. Homogeneous Media

3.1.2. Heterogeneous Systems (Confinement Effects)

3.2. Mechanisms of Parasitic Transport

3.2.1. Solvated Acidic Polymers

3.2.2. Oxides

4. Phenomenology of Transport in

Proton-Conducting Materials for Fuel-Cell

Applications

4.1. Hydrated Acidic Polymers

4.2. $\mathrm{PBI}-\mathrm{H}_{3} \mathrm{PO}_{4}$ Adducts

4.3. Heterocycle-Based Systems

4.4. Oxides

5. Recent Approaches toward New

Proton-Conducting Materials for Fuel-Cell Applications

6. Acknowledgement

7. References

\section{Introduction}

The el ectrolyte is the heart of any fuel cell. I deally, this component effectively separates the anode and cathode gases and/or liquids and mediates the electrochemical reaction occurring at the electrodes through conducting a specific ion at very high rates during the operation of the fuel cell. In other words,

\footnotetext{
* Author to whom correspondence should be addressed. E-mail address: kreuer@fkf.mpg.de.

₹ Max-Planck-Institut für Festkörperforschung

$\S$ University of Alabama in Huntsville.

\# Forschungszentrum J ülich.
}

transport through such electrolytes must be fast and highly selective; these two properties are frequently at odds with each other. Proton-conducting materials are used as the el ectrolyte for low- and intermediatetemperature fuel cells, which are currently attracting significant interest (i.e., polymer electrolyte membrane or proton exchange membrane fuel cells (PEMFCs)), direct methanol fuel cells (DMFCs), phosphoric acid fuel cells (PAFCs), and alkaline fuel cells (AFCs)). However, these are not true "single ion conductors", in that the transport phenomena are more complex, involving significant parasitic transport of other species. This review is concerned with the transport properties of presently available and emerging materials that have the potential to be used as the electrolyte for fuel cells soon. Strategies for the development of novel materials with transport properties approaching those of an "ideal separator", especially in the intermediate temperature range, are also discussed.

The last comprehensive review covering proton conductivity and proton conducting materials was written by one of the authors (dating back to 1996); ${ }^{1}$ since then, there have been several other review articles of similar scope (e.g., see Colomban²). There are also many reviews available on separator materials used for fuel cells (see articles in refs 3 and 4 and references therein, recent review-type articles, ${ }^{5-8}$ and a literature survey ${ }^{9}$ ), which, more or less, address all properties that are relevant for their functioning in a fuel cell. The transport properties are usually described in these articles; however, the treatments are frequently restricted to macroscopic approaches and handwaving arguments about the transport mechanisms. The purpose of the present review is to combine a few recently published results in the context of a discussion of transport phenomena in proton-conducting separator materials, which have some relevance in fuel cell applications (for a more complete list of the comprehensive literature in the field, the interested reader is referred to the aforementioned references).

Hence, the two main foci of this review are (i) the current understanding of the underlying el ementary processes and (ii) a phenomenological description of the resulting macroscopic transport phenomena. Because the first aspect comprises proton conduction mechanisms other than the mechanisms of "parasitic transport", this review may also be considered an 


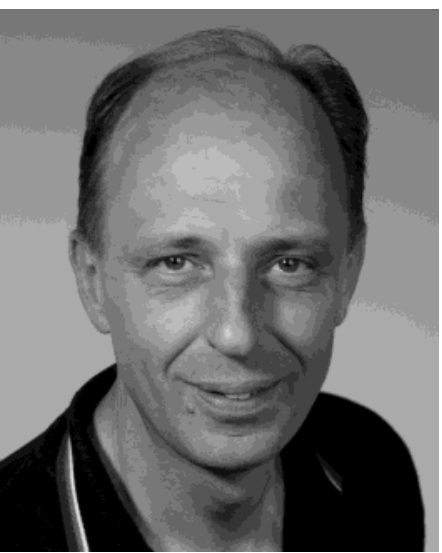

Klaus-Dieter Kreuer completed his Diploma Thesis on the "Distribution of $\mathrm{F}^{-}$and $\mathrm{OH}^{-}$in Hydroxofluoroapatites" in Mineralogy at the University of Cologne under the supervision of $F$. Freund in 1979. He received his Ph.D. from the University of Stuttgart (Department of Chemistry) in 1982. His thesis project in the department of A. Rabenau (Max-Planck-Institute for Solid State Research) involved the investigation of inorganic protonconducting compounds (e.g., layered minerals, zeolites), which showed the correlation of proton mobility and molecular diffusion (vehicle mechanism) for several cases. As a fellow of the "Studienstiftung des Deutschen Volkes", he benefited from the opportunity of a research stay at the California Institute of Technology (with the R. Vaughan group, 1978), and a Max-Planck award (Otto Hahn Medaille) allowed him to join the Massachussetts Institute of Technology (Department of Materials Science) as a visiting scientist (1983-1984). This period was part of his postdoctoral activities at the Max-Planck-Institute for Solid State Research, which were mainly concerned with research on alkaline-ion-conducting framework structures (such as lithium borate glasses and NASICON). From 1986 to 1992, he built up a research group for the development of chemical sensors $\left(\mathrm{pH}, \mathrm{O}_{2}\right)$ within a Swiss-German company (Endress \& Hauser). In 1992, he again joined the Max-Planck-Institute for Solid State Research, where he assisted J. Maier in building up his new department. Since 1990, he has been lecturing at the University of Stuttgart, from which he received his Habilitation Degree in 1999. His research interests are centered around the investigation of the formation and mobility of hydrogenderived defects in condensed matter. His work comprises materials preparation (inorganic and organic synthesis) and characterization (e.g., by nuclear magnetic resonance (NMR) and simulation techniques). The focus of his research is both a better understanding of fundamental issues and the development of novel materials for specific applications.

update on the corresponding part of ref 1 mentioned previously. The initial application of various simulation techniques to the investigation of fast elementary reactions as being part of proton conduction mechanisms had already summarized in this article. The early molecular dynamics simulation work of Münch et al. ${ }^{10}$ on the proton conduction mechanism in $\mathrm{CsHSO}_{4}$ diverse perovskite-type oxides and heterocycles (see Section 3.1.1) provided important insight into the conduction mechanism of model compounds, and some of the recent understanding of proton dynamics in water stems from simulation work (see Section 3.1.1.1). To date, simulation techniques, when appropriately combined with experimental results, establish insight into the path for the development of improved and new proton conductors. The increased availability of computational hardware, along with the improvement and development of new codes, haveled to a tremendous dissemination and increased impact of such techniques; hence, we have included a separate section that addresses current "simulation techniques" (Section 2), the results of which are included into the discussion of "transport mechanisms" (Section 3). The latter sec-

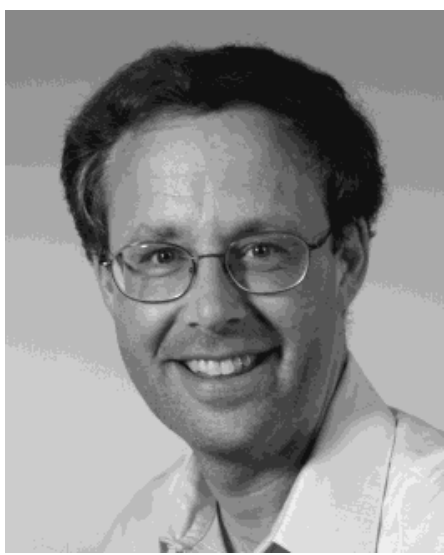

Stephen J. Paddison received a B.Sc.(Hon.) in Chemical Physics and a Ph.D. (1996) in Physical/Theoretical Chemistry from the University of Calgary, Canada. He was, subsequently, a postdoctoral fellow and staff member in the Materials Science Division at Los Alamos National Laboratory, where he conducted both experimental and theoretical investigations of sulfonic acid polymer electrolyte membranes. This work was continued while he was part of Motorola's Computational Materials Group in Los Alamos. He is currently an Assistant Professor in the Chemistry and Materials Science Departments at the University of Alabama in Huntsville, AL. Research interests continue to be in the development and application of first-principles and statistical mechanical methods in understanding the molecular mechanisms of proton transport in fuel-cell materials.

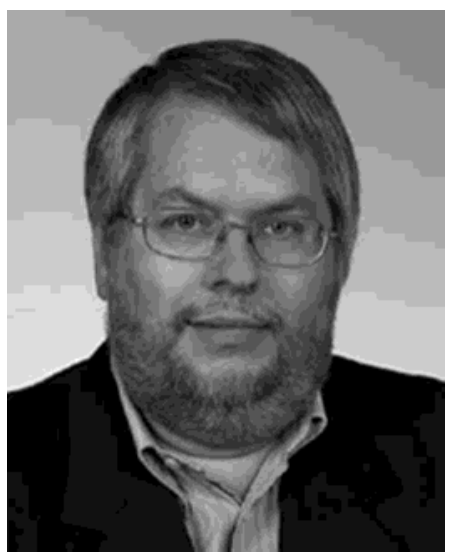

Eckhard Spohr received his diploma in Chemistry from the JohannesGutenberg-Universität in Mainz, Germany, in 1983. In 1986, he obtained his Ph.D. for computer simulation of water/metal interfaces at the MaxPlanck-Institut for Chemistry in Mainz. He continued to work for two years as a research associate in the aqueous simulation group of Karl Heinzinger, followed by a two-year visit to the University of California as a Feodor Lynen postdoctoral fellow of the Alexander von Humboldt Foundation, where he studied gas/surface reactions. In 1991, he moved to the Universität Ulm, where he obtained his habilitation degree in 1995 and worked as a University docent until 2000. In 2000, he moved to the Forschungszentrum Jülich, where he currently heads the physical chemistry group in the Institute for Materials and Processes in Energy Systems performing research on low-temperature fuel cells. His research interests center around computer simulations of aqueous electrolytes in confined environments. He teaches courses in theoretical chemistry at the Universität Ulm.

tion is truly cross-disciplinary in nature, because results from outside the fuel cell community are included. This is particularly true for the discussion of the proton mobility in homogeneous media (i.e., Section 3.1.1: water, aqueous solutions, phosphoric acid, imidazole, and simple cubic perovskites), which, in many cases, only constitute a single component of the heterogeneous fuel cell separator material. As a consequence of confinement and interaction of the 


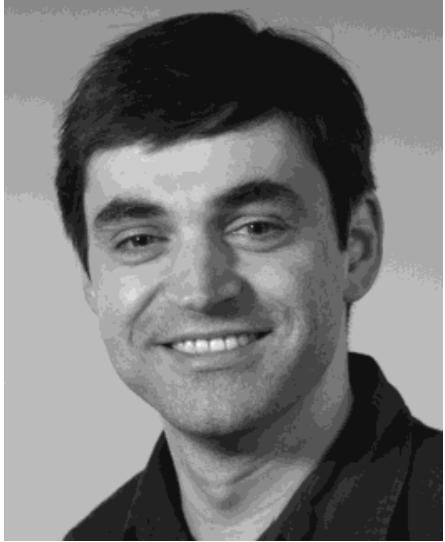

Michael Schuster studied chemistry at the Ludwig-Maximilians-Universität in Munich, Germany. He received his Ph.D. in Inorganic Chemistry under the direction of $A$. Schmidpeter and K. Karaghiosoff for his work on phosphates $\mathrm{P}_{m} \mathrm{X}_{n}{ }^{2-}(\mathrm{X}=\mathrm{S}$, Se, Te) and their NMR spectroscopic characterization. To continue his research interests in NMR spectroscopy, he moved to the Max-Planck-Institute for Solid State Research in Stuttgart in 2000 , where he is working on the development of proton-conducting materials for fuel cells. His studies are focused on the synthesis of imidazole-, sulfonic acid-, and phosphonic acid-based oligomers and polymers and their structural and dynamical properties. Characterization methods particularly include pulsed-field gradient and electrophoretic NMR spectroscopy.

mobile species, e.g., with the corresponding polymer matrixes, the transport properties of these heterogeneous systems are more complex and, therefore, are discussed in the section that follows (i.e., Section 3.1.2). Because most of this work has been done on prototypical materials, this discussion mainly involves readily available and extensively tested materials. However, the property relations may be easily transferred to the transport properties of related materials (Section 4) and may even provide a basis for the understanding of the transport properties of conceptually new proton-conducting separator materials (Section 5).

Apart from mechanistic aspects, we have also summarized the macroscopic transport behavior of some well-studied materials in a way that may contribute to a clearer view on the rel evant transport coefficients and driving forces that govern the behavior of such electrolytes under fuel cell operating conditions (Section 4). This also comprises precise definitions of the different transport coefficients and the experimental techniques implemented in their determination; providing a physicochemical rational behind vague terms such as "cross-over", which are frequently used by engineers in the fuel cell community. Again, most of the data presented in this section is for the prototypical materials; however, trends for other types of materials are also presented.

Thus, the aim of this review is to give the currently best possible generally applicable understanding of mass and charge transport in fuel-cell-relevant proton conductors, including a description of the fundamental underlying elementary reactions for these transport processes and quantitative phenomenol ogical descriptions of transport in these materials. The first is important for further adaptation of available electrolytes to specific fuel cell conditions or, subsequently, the development of new materials. The latter allows a better understanding of the transient and steady-state behavior of known materials under the varying conditions of an operating fuel cell. Access to the numerous references providing transport data of proton-conducting fuel cell separators may be found in the corresponding chapters of refs 3 and 4 . The referencing of this review is restricted to those papers that are relevant for the more-general picture. Of course, this also comprises extensive crossreferencing to the non-fuel-cell literature.

Before proceeding to this, let us briefly mention a few general directions in recent fuel cell research for which the transport properties of the proton-conducting electrolyte are essential. Much of the current research is on proton exchange membrane (PEM) fuel cells where the electrolyte is DuPont's Nafion or some other sulfonated polymer. ${ }^{5}$ Such polymers naturally combine, in one macromolecule, the high hydrophobicity of the backbone with the high hydrophilicity of the sulfonic acid functional group, which results in a constrained hydrophobic/hydrophilic nanoseparation. The sulfonic acid functional groups aggregate to form a hydrophilic domain that is hydrated upon absorption of water. It is within this continuous domain that ionic conductivity occurs: protons dissociate from their anionic counterion $\left(-\mathrm{SO}_{3}{ }^{-}\right)$and become solvated and mobilized by the hydration water. Water typically must be supplied to the el ectrolyte through humidification of the feed gases and is also produced by the electrochemical reduction of oxygen at the cathode. This is the reason for two serious problems relevant for the use of such membranes in fuel cells: (i) because high proton conductivity is only obtained at high levels of hydration, the maximum operation temperature is approximately limited to the condensation point of water (i.e., 100 ${ }^{\circ} \mathrm{C}$ for a water pressure of 1 atm $\left.\left(10^{5} \mathrm{~Pa}\right)\right)$; and (ii) any protonic current also leads to transport of water through the membrane (as a result of el ectroosmotic drag) and, if methanol dissolves in the membrane, this is transported virtually at the same rate. The limited operating temperature and the acidity of the electrolyte makes it necessary to use platinum or platinum alloys (the most active but also the most expensive electrocatalyst) to promote the electrochemical reactions in the anode and cathode structures. However, even with platinum, only rather pure hydrogen can be oxidized at sufficient rates. At the operation temperature of state-of-the-art PEM fuel cells, the rate of direct oxidation of methanol (which is frequently considered an environmentally friendly fuel) is not sufficiently high, and, hence, even trace amounts of $\mathrm{CO}$ present in any hydrogen-rich reformate (e.g., produced by steam reforming of methanol or methane) poison platinum-based catalysts through adsorption thus blocking the reaction sites. The humidification requirements, along with the high electro-osmotic drag of water and methanol in conventional membranes, complicate the water and heat management of the fuel cell and lead to a significant chemical short-circuiting, i.e., parasitic chemical oxidation of methanol at the cathode.

Therefore, tremendous engineering effort has been expended to at least control the fluxes of water and methanol in such a way that the resulting transient 
and steady-state concentration profiles of these species across the membrane still permit acceptable function of the membrane and the electrode structures. The fact that measured water concentration profiles $^{11}$ differed substantially from the results of early membrane modeling ${ }^{12,13}$ made it clear that the transport behavior of such membranes were not fully understood. It was the availability of better data and improved phenomenological models concerning the macroscopic transport in such membranes ${ }^{14}$ that helped to better describe the behavior of available membranes under fuel cell conditions as a function of the boundary conditions (i.e., current, gas humidification, etc.) and membrane properties (e.g., thickness). In addition, there have been many attempts to modify the transport properties, especially by forming composites with highly dispersed inorganic phases. More radical approaches aim at conceptually different separator material exclusively transporting protons and being able to operate at higher temperatures in a low humidity environment. Such a "dream membrane" is widely considered to be the key to further progress in "low"-temperature fuel cell technology: higher operation temperatures would supersede or simplify gas conditioning and purification, reduce the precious metal loading (along with the problems of heat rejection), while reducing hydration requirements may avoid the need to pressurize the system. Because these aspects are all related to the cost of the fuel cell system, which is increasingly attracting attention, understanding the transport properties as a function of molecular structure and morphology and development of new separator materials are the focal points in current fuel cell research.

Whether future progress will be achieved "within the box" by modifying available materials or by designing conceptually different materials is not yet clear. In any case, a better understanding of the mechanisms governing transport of the various species in the separator materials is useful in the effort of further materials research and development.

\section{Theoretical Methodologies and Simulation Tools}

This section briefly describes some of the theoretical methods and types of simulations that have recently been applied to understand the structural and dynamical features of transport in proton conductors. Although the transport properties and, hence, mechanisms are strongly correlated to the morphology of the material, theoretical studies of the morphology will not be discussed here.

Quantum chemistry or molecular electronic structure theory is the application of the principles of quantum mechanics to cal culate the stationary states of molecules and the transitions between these states. Today, both computational and experimental groups routinely use ab initio (meaning "from first principles") molecular orbital calculations as a means of understanding structure, bonding, reaction paths between intermediates etc. Explicit treatment of the electrons means that, in principle, one does not make assumptions concerning the bonding of a system, which allows "surprises" to be witnessed. Thus, it is a valuable adjunct to experimental work becoming another powerful tool for the chemist, molecular physicist, and material scientist.

Fundamental information concerning local structure, molecular hydrophilicity, aggregation of ionic groups through the formation of hydrogen bonds, and proton dissociation of acidic groups in the fragments of PEMs has been obtained through the determination of global minimum (i.e., equilibrium) energy structures. ${ }^{15-25}$ Because these techniques explicitly treat all the electrons of the system to obtain the el ectronic structure, only fragments of polymer have been studied, where the system size has typically been limited to $<100$ atoms.

The dynamics of particles (i.e., atoms, molecules, ions, etc.) may be investigated through the continuous (i.e., time-evolving) solution of Newton's equations (i.e., classical molecular dynamics (MD)) or with stochastic methods including Monte Carlo (MC) theory. Classical MD simulations with empirical potentials can handle systems consisting of thousands of particles over time periods of nanoseconds and have been used to study proton transport in materials as a function of parameters such as temperature, water content, and, in polymers, equivalent weight and chemical and physical characteristics of main and side chains. ${ }^{26-29}$ To address continuous changes in valence bond networks during the transport of protons, empirical valence bond (EVB) interaction potentials have been devised. ${ }^{30-32} \mathrm{Ab}$ initio molecular dynamics (AIMD) simulations have successfully been used to study the proton dynamics in homogeneous systems (e.g., water ${ }^{33-36}$ and imidazole $^{37}$ ), and recently even more complex and, therefore, extended "model" systems have been examined (e.g., trifluoromethanesul fonic acid monohydrate ${ }^{38,39}$ ). They are computationally very demanding if "meaningful" trajectories of only tens of picoseconds are to be sampled. The payoff in such calculations is the determination of completely new and potentially significant insight into molecular mechanisms. The recent AIMD study of trifluoromethanesulfonic acid monohydrate solid ${ }^{38,39}$ reveal ed the very first evidence of the possible role of the sulfonate anions and Zundel ion in proton transfer in minimally hydrated PEMs.

Microscopic el ectrolyte theory (Section 2.4) based on a statistical mechanical framework has recently been applied to study the diffusion of protons in a single hydrated channel or pore in several PEMs. ${ }^{40-46}$ This approach makes several assumptions concerning the pore geometry, distribution of fixed anionic groups, and, primarily, the vehicular mechanism of proton transport through the center of the pore. However, this model has been able to compute the proton self-diffusion coefficient in both $\mathrm{N}$ afion and S-PEEK (sulfonated poly(arylene ether ether ketone)) membranes correctly over a significant range of hydration without resorting to any fitting parameters, requiring only membrane-specific morphology information from small-angle X-ray scattering (SAXS) experiments and structural information from electronic structure calculations. 
Confinement of water into regions with dimensions of only a few nanometers, such as typically those found in PEMs, accompanied by a strong el ectrostatic field due to the anions, will result in a significantly lower dielectric constant for the water than that observed in bulk water. Measurement of this structural ordering of the water has not been accomplished experimentally to date, and this was the motivation to the recent calculation of the dielectric saturation of the water in PEMs with an equilibrium thermodynamical formulation. ${ }^{4-51}$ In addition to information concerning the state of the water this modeling has provided information concerning the distribution of the dissociation protons in sulfonic acid-based PEMs.

The various methodologies are discussed in somewhat more detail in the following sections.

\subsection{Ab Initio Quantum Chemistry}

Only a very concise description of quantum chemistry is presented in this section; the reader is referred to standard texts for a more complete treatment. ${ }^{52-54}$ The aim of quantum chemistry or ab initio el ectronic structure theory is the solution of the time-independent Schrödinger equation:

$$
\mathrm{H}(\mathrm{r} ; \mathbf{R}) \Psi(\mathrm{r} ; \mathbf{R})=\mathrm{E}(\mathbf{R}) \Psi(\mathrm{r} ; \mathbf{R})
$$

This yields the molecular wave function, $\Psi(r ; \mathbf{R})$, which is dependent explicitly on the $3 n$ coordinates of all $\mathrm{n}$ electrons (denoted collectively as $\mathbf{r}$ ) and implicitly on the coordinates of all A nuclei (denoted collectively as $\mathbf{R}$ ); and the (total) molecular energy, $E(\mathbf{R})$, which parametrically is dependent only on the nuclear positions. The distinction in functional dependence of these quantities is due to the BornOppenheimer (BO) approximation, ${ }^{55}$ which separates (slow) nuclear motion from (fast) electronic motion. It is through determination of $E(\mathbf{R})$ that the potential energy surface is defined. The total energy or molecular electronic Hamiltonian, $\mathrm{H}(\mathbf{r} ; \mathbf{R})$, is an operator consisting of the kinetic energy of all the electrons and the potential energy due to the following forces: electron-nuclear attraction, el ectron-el ectron repulsion, and nuclear-nuclear repulsion. I ts explicit form in atomic units is

$$
\begin{aligned}
& H(r ; R)=-\frac{1}{2} \sum_{i}^{n}\left(\frac{\partial^{2}}{\partial \mathbf{x}_{i}^{2}}+\frac{\partial^{2}}{\partial \mathbf{y}_{i}^{2}}+\frac{\partial^{2}}{\partial \mathbf{z}_{i}^{2}}\right)- \\
& \sum_{i}^{n} \sum_{\alpha}^{A} \frac{Z_{\alpha}}{\left|r_{i}-\mathbf{R}_{\alpha}\right|}+\frac{1}{2} \sum_{i}^{n} \sum_{j}^{n} \frac{1}{\left|r_{i}-r_{j}\right|}+\frac{1}{2} \sum_{\alpha}^{A} \sum_{\beta}^{A} \frac{Z_{\alpha} \mathbf{Z}_{\beta}}{\left|\mathbf{R}_{\alpha}-\mathbf{R}_{\beta}\right|}
\end{aligned}
$$

This partial differential equation with 3n unknowns is impossible to solve exactly (beyond the hydrogen molecule) and, thus, various approximations are generally made.

In a first class of approximations, the solution is sought of a simpler set of equations rather than the exact equations. Under the Hartree-Fock (i.e., HF) approximation, 56,57 the function of $3 n$ variables is reduced to $\mathrm{n}$ functions, which are referenced as molecular orbitals (MOs), each dependent on only three variables. Each MO describes the probability distribution of a single electron moving in the average field of all other electrons. Because of the requirements of antisymmetry, with respect to the interchange of any two el ectrons, and indistinguishability of electrons, the trial wave function is a single determinantal wave function of the MOs. The optimum MOs are determined through variationally minimizing $E(\mathbf{R})$. Thus, the $\mathrm{HF}$ method is a meanfield method applied to the many-electron problem, the solution of which is commonly referred to as the self-consistent field (SCF). Because HF theory does not include details of the instantaneous electronelectron correlations, several post-HF techniques have been developed, including many-body (or MøllerPlesset) perturbation theory, ${ }^{58}$ and configuration interaction (CISD). ${ }^{59}$ In the former method, a "zeroorder" description of the ground-state wave function is assumed to be a determinantal wave function constructed from the HF MOs upon which a perturbation acts that the difference between the sum of Fock operators and the exact Hamiltonian. In CISD, electron-pair correlations are treated self-consistently through the assumption that the wave function is a linear combination of the HF determinant with all determinants formed by single and double orbital substitutions of coefficients determined variationally.

A second approximation that is commonly invoked in all these methods is the expansion of the unknown MOs in terms of a given, fixed, and finite set of functions. These functions are usually referenced as the atomic orbital (AO) basis set, where the atomic orbitals are usually expanded through linear combinations of Gaussians, because of the fact that all required matrix elements may be evaluated analytically. ${ }^{60}$ The basis sets may be "minimal" (i.e., one basis function per atomic orbital), "split valence" (two basis functions per valence atomic orbital, one per core orbital), or higher zeta (where n-zeta means $n$ basis functions per orbital). Multiple basis functions per atomic orbital allow the size of the orbitals to increase (i.e., along a bond axis) or decrease (i.e., perpendicular to a bond axis). The higher-order basis sets may also be augmented with polarization functions (e.g., one or more sets of $d$ functions on firstrow atoms) that describe small displacements of the orbitals from atomic centers in the molecular environment, and diffuse functions for anions and Rydberg excited states. The size of the Hamiltonian to be diagonalized is proportional to $\mathrm{N}^{4}$ (where $\mathrm{N}$ is the number of AOs); therefore, the use of large basis sets is very time-consuming.

The majority of the molecular-scale information concerning the effects of structure and local chemistry on proton dissociation and separation in PEM fragments alluded to previously ${ }^{15-23}$ were initially determined using HF theory and split valence local basis sets. Refinements to the equilibrium configurations were made using both Møller-Plesset (MP) perturbation schemes and hybrid density functional theory (described below).

A widely used alternative to the electron correlation treatments mentioned previously is the density 
functional theory (DFT), ${ }^{61}$ which seeks to determine the exact ground state energy and electron density directly, without computing a many-electron wave function. The electron density is only a function of three variables (unlike the wave function, which is dependent on $3 n$ variables); thus, DFT dramatically simplifies the cal culations and, therefore, has become the preferred method for treating large molecules. DFT relies on two fundamental theorems. ${ }^{62}$ The first states that the ground-state electron density, $n(\mathbf{r})$, uniquely determines the external potential $\mathbf{v}(\mathbf{r})$ and, thus, given the nuclear coordinates, determines the ground-state energy, $E_{v(r)}[n(r)]$, and all properties of the ground state, i.e.,

$$
\begin{array}{r}
E_{v(r)}[n(r)] \equiv \int v(r) n(r) d r+F[n(r)] ; F[n(r)] \equiv \\
(\Psi[n(r)],(T+U) \Psi[n(r)])
\end{array}
$$

where $F[n(\mathbf{r})]$ is the desired (unknown) functional, and $T$ and $U$ are the kinetic and potential energies, respectively. The second states that, given the functional, it is the one that minimizes the energy, thereby providing a variational principle to determine the density, i.e.,

$$
E_{v(r)}[n(r)] \geq E_{v(r)}\left[n_{0}(r)\right] \equiv E
$$

$\mathrm{F}[\mathrm{n}(\mathrm{r})]=$

$$
T_{s}[n(r)]+\frac{1}{2} \int \frac{n(r) n\left(r^{\prime}\right)}{\left|r-r^{\prime}\right|} d r d r^{\prime}+E_{x c}[n(r)]
$$

where the first term $\left(T_{s}[n(r)]\right)$ is the kinetic energy, the second term the classical Coulombic repulsion energy, and the last term $\left(E_{x c}[n(r)]\right)$ the exchange correlation energy. The challenge in DFT is the design of accurate functionals, and, specifically, the difficulties lie in determining $T_{s}[n(r)]$ and $E_{x c}[n(r)]$. Considerable progress in constructing a kinetic energy density functional came through the reformulation of DFT by Kohn and Sham, termed Kohn-Sham (KS) density functional theory. ${ }^{63}$ In KS-DFT, an artificial reference system is constructed that consists of noninteracting electrons, which has exactly the same electron density as the real molecular system of interacting electrons. The kinetic energy is approximated as that of the noninteracting reference system, which can be exactly evaluated in terms of the Kohn-Sham orbitals $\varphi_{\mathrm{j}}$. The self-consistent set of $\mathrm{KS}$ equations is

$$
\begin{gathered}
\left(-\frac{1}{2} \nabla^{2}+v(r)+\int \frac{n\left(r^{\prime}\right)}{\left|r-r^{\prime}\right|} d r^{\prime}+v_{x c}(r)-\epsilon_{j}\right) \varphi_{j}(r)=0 \\
n(r)=\sum_{j=1}^{N}\left|\varphi_{j}(r)\right|^{2} \\
v_{x c}(r)=\delta E_{x c} \frac{[n(r)]}{\delta n(r)} \\
E=\sum_{1}^{v} \epsilon_{j}-\int \frac{n(r) n\left(r^{\prime}\right)}{\left|r-r^{\prime}\right|} d r d r^{\prime}-\int v_{x c}(r) n(r) d r+ \\
E_{x c}[n(r)](6)
\end{gathered}
$$

where the final equation is the expression for the ground-state energy of the molecular system. These equations differ from the Hartree equations only by the inclusion of the exchange-correlation potential $v_{x c}(r)$. Furthermore, if the exchange correlation energy is partitioned into separate contributions due to correlation and exchange, i.e., $E_{x c}[n(r)]=E_{x}+E_{c}$, and only the correlation energy neglected, then the treatment becomes a variant of HF theory. The simplest approximation for $E_{x c}[n(r)]$ is the generally accepted local density approximation (LDA):63

$$
E_{x c}^{L D A}[n(r)] \equiv \int \epsilon_{x c}(n(r)) n(r) d r
$$

where $\epsilon_{\mathrm{xc}}(\mathrm{n})$ is the exchange-correlation energy per particle of a uniform interacting electron gas of density $\mathbf{n}$. The KS orbitals in the LDA are surprisingly close to HF orbitals, although DFT-LDA calculations neglect the nonlocal nature of exchange terms that are typical for the HF method. The next level of approximations is the generalized gradient approximation (i.e., GGA):

$$
\mathrm{E}_{\mathrm{XC}}^{\mathrm{GGA}} \equiv \int \mathrm{f}(\mathrm{n}(\mathrm{r}),|\nabla \mathrm{n}(\mathrm{r})|) \mathrm{dr}
$$

in which $\mathrm{f}(\mathrm{n},|\nabla \mathrm{n}|)$ is a suitably chosen function of its two variables. Popular correlation GGAs include those of Lee, Yang, and Parr, ${ }^{64}$ Perdew (1986), ${ }^{65}$ and Perdew and Wang (1991). ${ }^{66}$ These gradient-corrected correlation functionals in combination with the Becke exchange functional67 (based on considering the exchange energies for rare gases, in addition to the known behavior for the uniform electron gas), typically yield accurate relative energies and good thermochemistry. 68

As a consequence of the size limitations of the $a b$ initio schemes, a large number of more-approximate methods can be found in the literature. Here, we mention only the density functional-based tight binding (DFTB) method, ${ }^{69-72}$ which is a two-center approach to DFT. The method has been successfully applied to the study of proton transport in perovskites $^{73}$ and imidazole ${ }^{37}$ (see Section 3.1.1.3). The fundamental constraints of DFT are (i) treatment of excited states and (ii) the ambiguous choice of the exchange correlation function. In many cases, the latter contains several parameters fitted to observable properties, which makes such calculations, in fact, semiempirical.

\subsection{Molecular Dynamics}

Classical molecular dynamics (MD) implementing predetermined potentials, either empirical or derived from independent electronic structure calculations, has been used extensively to investigate condensedmatter systems. ${ }^{74}$ An important aspect in any MD simulation is how to describe or approximate the interatomic interactions. Usually, the potentials that describe these interactions are determined a priori and the full interaction is partitioned into two-, three, and many-body contributions, long- and shortrange terms, etc., for which suitable analytical functional forms are devised. ${ }^{75}$ Despite the many successes with classical MD, the requirement to devise fixed potentials results in several serious problems 
in systems where distinct atoms or molecules cause a myriad of different interatomic interactions that should be parametrized or where the electronic structure or bonding pattern changes qualitatively in the course of the simulation. ${ }^{76,77}$ These drawbacks have been overcome by the various techniques of $a b$ initio molecular dynamics (AIMD). An overview of a few of the various MD schemes implemented in the study of proton conduction is presented in the following sections.

\subsubsection{Classical Molecular Dynamics and Monte Carlo Simulations}

Atomistic computer simulations are a statistical mechanical tool to sample configurations from the phase space of the physical system of interest. The system is uniquely treated by specifying the interactions between the particles (which are usually described as being pointlike), the masses of all the particles, and the boundary conditions. The interactions are calculated either on-the-fly by an electronic structure cal culation (see Section 2.2.3) or from potential functions, which have been parametrized before the simulation by fitting to the results of electronic structure calculations or a set of experimental data. In the first case, one frequently speaks of AIMD (see Section 2.2.3), although the motion of the nuclei is still treated classically.

Having specified the interactions (i.e., the model of the system), the actual simulation then constructs a sequence of states (or the system trajectory) in some statistical mechanical ensemble. Simulations can be stochastic (M onte Carlo (MC)) or deterministic (MD), or they can combine elements of both, such as forcebiased MC, Brownian dynamics, or generalized Langevin dynamics. It is usually assumed that the laws of classical mechanics (i.e., Newton's second law) may adequately describe the atoms and molecules in the physical system.

The MC scheme was first published by Metropolis et al. ${ }^{78}$ in 1953 and applied to the calculation of the equation of state of a simple hard-sphere liquid. Each configuration in an MC simulation is generated stochastically in such a way that the molecular configuration is dependent only on the previous configuration. The MC method is often performed in the canonical ensemble, i.e., for a fixed number of molecules $\mathrm{N}$ placed in a fixed volume $\mathrm{V}$ and maintained at a constant temperature T. However, many variants of the method exist (see, e.g., ref 74). During the MC simulation, configurations are generated in such a way that, after many configurations have been obtained, each configuration occurs approximately with the appropriate probability of the canonical ensemble, given by the Boltzmann factor $\exp [-E(\mathbf{R}) /$ $(k T)$ ], where $E(\mathbf{R})$ is now the interaction potential, and $\mathrm{k}$ the Boltzmann constant.

The MD method was first used by Alder and Wainwright. ${ }^{79}$ In the standard MD scheme for equilibrium systems, the positions $\mathbf{r}_{\mathbf{i}}$ of atom $\mathrm{i}$ are obtained by solving Newton's equations of motion:

$$
m_{i} \ddot{r}_{i}=F_{i}=-\nabla_{i} E(R)
$$

where $m_{i}$ is its mass and the force $F_{i}$ is the negative gradient of the potential energy $E(\boldsymbol{R})$, with respect to $\mathrm{i}$. The MD scheme leads (contrary to the MC scheme) to a time-correlated sequence of configurations (trajectory), which can be analyzed to calculate dynamic properties of the system.

The potential energy is often written as a sum of pairwise additive interactions. Frequently, Coulombic interactions between partially charged atoms and additional interaction functions that describe shortrange repulsion due to exchange-correlation effects and long-range disperse attraction are used as ingredients in the interatomic potentials. In most cases, the latter interaction function is of the Lennard-J ones type. Molecular geometry and connectivity is maintained by specifying harmonic or Morse-type stretching interactions along a chemical bond, and anglebend interactions for valence angles and torsion interactions to maintain molecular conformations. Thus, a typical simple force field is the sum over all pairs of Coulombic and short-range interactions, all bonds, all valence angles, and all dihedral angles in a molecule. More complex force fields can contain more elaborate terms and couplings between them. Many-body induction interactions are often included in the interaction energy $E(\mathbf{R})$ by introducing fluctuating point dipoles or fluctuating point charges, whose value is self-consistently determined through the instantaneous electric field and the atomic or molecular polarizabilities. An alternative is the introduction of extra charged particles in a shell model (SM). ${ }^{80}$

Typical numbers of atoms range over the order of several hundred to several tens of thousands, which are located in a regular cell of volume $\mathrm{V}$. The cell and the particles are replicated infinitely in one, two, or three directions of space, depending on whether a cylindrical or other one-dimensional system, a slab system with two external interfaces, or a bulk system is to be simulated. These periodic boundary conditions avoid undesired surface effects beyond those that one explicitly wishes to study. The cells are open and particles can move freely from one cell to the next. For each particle leaving the cell, one of its replicas enters the cell; therefore, the overall particle number in the cell, and thus the density, remains constant. Because the number of interactions to be calculated in such a system is infinitely large, shortrange interactions (such as the Lennard-J ones term) need to be smoothly truncated, and lattice summation methods (such as the E wald method) are implemented for the treatment of long-range forces.

The theory of statistical mechanics provides the formalism to obtain observables as ensemble averages from the microscopic configurations generated by such a simulation. From both the MC and MD trajectories, ensemble averages can be formed as simple averages of the properties over the set of configurations. From the time-ordered properties of the MD trajectory, additional dynamic information can be calculated via the time correlation function formalism. An autocorrelation function $c_{a a}(t)=\langle a(\tau)$ - $\mathrm{a}(\mathrm{t}+\tau)\rangle$ is the ensemble average of the product of some function a at time $\tau$ and at a later time $t+\tau$. 
In an equilibrium system, all times $t$ are equivalent and can thus be averaged over. Transport coefficients can be calculated as integrals over these functions, e.g.,

$$
D=\frac{1}{3} \int_{0}^{\infty} c_{w v} d t=\lim _{x \rightarrow \infty} \frac{\left\langle[r(t+\tau)-r(\tau)]^{2}\right\rangle}{6 t}
$$

The diffusion coefficient $D$ is one-third of the time integral over the velocity autocorrelation function $c_{v v}(t)$. The second identity is the so-called Einstein relation, which relates the self-diffusion coefficient to the particle mean square displacement (i.e., the ensemble-averaged square of the distance between the particle position at time $\tau$ and at time $\tau+\mathrm{t}$ ). Similar relationships exist between conductivity and the current autocorrelation function, and between viscosity and the autocorrelation function of elements of the pressure tensor.

\subsubsection{Empirical Valence Bond Models}

A standard classical force field is able to describe conformational changes of molecules. However, it is generally unable to describe the formation and breaking of chemical bonds. The reason is that the atoms participating in the stretch, bend, and torsion terms of the force field need to be specified initially. Changing this specification during the simulation leads to non-Hamiltonian behavior, which makes the simulation data unusable for analysis. Elegant solutions to this problem are ab initio simulation schemes (see Section 2.2.3), which naturally describe the change of chemical bonding via the instantaneous calculation of the electronic structure. In many situations, this scheme is computationally too expensive to be used. Thus, the need arises to develop empirical potential functions that (i) allow the change of the valence bond network over time and (ii) are simple enough to be used efficiently in an otherwise classical simulation code. For dissociation reactions, one possibility is the use of interaction potential functions with the proper asymptotic behavior. As an example, consider the water molecule. In practically all-empirical water models, $\mathrm{O}$ and $\mathrm{H}$ atoms carry (fixed) partial charges. Thus, these models are incapable of describing the autodissociation of water. For a model that describes the dissociation into protons and hydroxyl ions, full ionic charges need to be placed on the atoms (i.e., +e for the proton, -2efor oxygen). Such Coulombic interactions are too strong at short and intermediate range; thus, an additional potential function must be constructed. ${ }^{81,82}$ An alternative is the valence bond (VB) method, where the chemical bond in a dissociating molecule is described as the superposition of two states: a less-polar bonded state and an ionic dissociated state. Unlike VB theory in quantum mechanics, the matrix elements of the Hamiltonian are not calculated on an el ectronic basis (see Section 2.1) but by empirical force fields.

As an example, proton transfer in an $\mathrm{H}_{5} \mathrm{O}_{2}{ }^{+}$complex may be described as a superposition of two states, namely $\mathrm{H}_{2} \mathrm{O}-\mathrm{H} \cdots \mathrm{OH}_{2}$ and $\mathrm{H}_{2} \mathrm{O} \cdots \mathrm{H}-\mathrm{OH}_{2}$ (where the solid line (-) describes a chemical bond and the dotted line $(\cdots)$ is a hydrogen bond). The energy of each state is calculated from empirical force field terms for intramolecular hydronium interactions, intramolecular water interactions, and the intermolecular water-hydronium interactions, yielding the energies in state 1 and 2: $\mathrm{H}_{11}(\mathbf{R})$ and $\mathrm{H}_{22}-$ $(\mathbf{R})$, which are generally nonidentical. By further specifying empirical coupling functions $\mathrm{H}_{12}(\mathbf{R})=\mathrm{H}_{21^{-}}$ (R) as functions of the set of particle coordinates $\mathbf{R}$, the compound states can be calculated via diagonalization of the $2 \times 2$ Hamiltonian matrix. This method is called the empirical valence bond (EVB) method and was first introduced by Warshel and Weiss. ${ }^{30-32}$ $H 2(R)$ can be adjusted to reproduce experimental data or ab initio potential energy surfaces. Approximating the motion of protons as classical on the (time-dependent) ground-state potential energy surface, a viable MD scheme can be developed. The procedure is analogous for larger clusters with more basis states, where a larger matrix must be diagonalized. The ground state of the system is specified by the eigenvector of the lowest-energy eigenvalue.

Simultaneously, Borgis and Vuilleuimier ${ }^{83-87}$ and Voth and co-workers ${ }^{88-93}$ developed multistate EVB models for proton transport in aqueous solution. In their models, a protonated cluster involving $n$ water molecules, $\mathrm{H}_{2 n+1} \mathrm{O}_{n}{ }^{+}$, is described by $n$ zeroth-order VB states. In each of these states, the proton defect is formally located on one of the $\mathrm{n} \mathrm{O}$ atoms (i.e., one of the $\mathrm{n} \mathrm{O}$ atoms forms three bonds). At any time t, the ground state is calculated via matrix diagonalization; partial charges (and possibly other parameters of the force field) are reassigned to the complex according to the eigenvector. When the proton defect diffuses structurally through this cluster, the contribution of some states can become negligibly small (because the $\mathrm{O}$ atom is too far from the proton defect). It is then possible to remove these water molecules from the cluster and replace them by others, which are closer to the proton defect but do not yet interact with the cluster. Thus, over time, the composition of the proton cluster can change and proton transport is possible. The multistate EVB models were used to investigate the structure of protonated complexes in aqueous solutions, proton transport dynamics, the relaxation of the hydrogen-bonded environment, and the role of the quantum nature of proton motion for structure and dynamics. 86,89

Based on these experiences, Walbran and Kornyshev $^{94}$ developed a much simpler two-state EVB model. Their model is designed in such a way that it can be used (i) when treating protons classically and (ii) for systems with high proton concentrations. The rationale behind its development was to eventually investigate proton transport in polymer electrolyte membranes. However, in this initial work, they studied only proton mobility in pure water. The ground-state energy is calculated, as for the other EVB models, from the lowest eigenvalue, in this simple case, according to

$$
\mathrm{E}=-\frac{1}{2}\left(\mathrm{H}_{11}+\mathrm{H}_{22}+\sqrt{\mathrm{H}_{11}^{2}+\mathrm{H}_{22}^{2}+4 \mathrm{H}_{12}^{2}}\right)
$$

What distinguishes it from the multistate models is the fact that the partial charges on the atoms are 
not determined from the eigenvectors of the ground state but through a charge-switching function, which, in turn, is dependent only on the coordinates of the Zundel complex. Thus, Coulombic interaction (and other time-consuming interactions) can be calculated using the adiabatic (effective) charges. In this way, the calculation of Coulombic interactions is substantially simplified; furthermore, the diagonalization of the individual protonated complexes decouples and can be easily performed, even at water-to-proton ratios on the order of 5-10, for which the use of the multistate EVB models with 10 or more basis states would be impossible when more than one proton is present. The model parameters were fit to reproduce the structure and formation energies of small protonated clusters. Because of the limitation of treating only two VB states and limitations in the parameter choice mandated by the requirement that the model needs to remain Hamiltonian in nature and simultaneously allow proton transport, the model usually underestimates the mobility of excess protonic defects and overestimates the self-diffusion coefficient of water, whereas the temperature dependence is usually close to the experimental values. This two-state EVB model was recently applied to simulations of "model" polymer el ectrolyte membranes (see Section 3.1.2.). ${ }^{26-29}$

\subsubsection{Ab Initio Molecular Dynamics (AIMD)}

The basic underlying methodology in AI MD is the computation of the forces acting on the nuclei from electronic structure cal culations (see previous discussion) that are performed "on the fly" as the trajectory of the molecules is generated. Thus, the electronic variables are not integrated out beforehand but are active degrees of freedom. This implies that, given a suitable approximate solution of the many-electron problem, "chemically complex" systems may be treated with AIMD. Furthermore, the approximation is shifted from the level of selecting the appropriate potentials to the level of choosing a particular approximation for solution of the Schrödinger equation. Despite these advantages, however, there is a price to be paid in AIMD: the correlation lengths and relaxation times that are accessible are much smaller than what is typically accessi ble via classical MD. Of the various AIMD techniques, we will restrict our discussion in this review to only two methods: (a) Born-Oppenheimer (BO) molecular dynamics and (2) Car-Parrinello molecular dynamics (CPMD).

In BO AIMD, the static electronic structure is solved at each MD time step, given the set of fixed nuclear positions at that instance in time. Thus, the problem is reduced to solving a time-independent quantum problem concurrently to propagating the nuclei via Newton's second law. Therefore, the BO method is defined by

$$
\begin{gathered}
\mathrm{M}_{\mathrm{i}} \ddot{\mathbf{R}}_{\mathrm{i}}(\mathrm{t})=-\nabla_{\mathrm{i}} \min _{\Psi_{0}}\left\{\left\langle\Psi_{0}\left|\mathrm{H}_{\mathrm{e}}\right| \Psi_{0}\right\rangle\right\} \\
\mathrm{E}_{0} \Psi_{0}=\mathrm{H}_{\mathrm{e}} \Psi_{0}
\end{gathered}
$$

for the electronic ground state. It is important to realize that the minimum of $\left\langle\mathrm{H}_{\mathrm{e}}\right\rangle$ must be reached at each BO MD step, which may be solved (for example) under either the HF approximation or with KS-DFT (see previously given brief method descriptions). A commonly used technique implementing the latter electronic structure approach is the VASP totalenergy code. ${ }^{95-97}$

The Car-Parrinello approach ${ }^{98}$ to AIMD exploits the quantum mechanical adiabatic time-scale separation of fast electronic and slow nuclear motion by transforming that into adiabatic energy scale separation in the framework of dynamical systems theory. This is achieved through mapping the two-component quantum/classical problem onto a two-component purely classical problem with two separate energy scales at the expense of loosing the explicit time dependence of the quantum subsystem dynamics. The CPMD method makes use of the following classical Lagrangian:

$\mathrm{L}_{\mathrm{CP}}=\sum_{\mathrm{i}} \frac{1}{2} \mu_{\mathrm{i}}\left\langle\dot{\psi}_{\mathrm{i}} \mid \dot{\psi}_{\mathrm{i}}\right\rangle+\frac{1}{2} \sum_{\mathrm{i}} \mathrm{M}_{\mathrm{i}} \ddot{\mathbf{R}}_{\mathrm{i}}^{2}-\mathrm{E}\left[\left\{\psi_{\mathrm{i}}\right\},\left\{\mathbf{R}_{\mathrm{i}}\right\}\right]$

to generate trajectories for the ionic and electronic degrees of freedom via the coupled set of equations of motion:

$$
\begin{gathered}
\mathrm{M}_{\mathrm{i}} \ddot{\mathbf{R}}_{\mathrm{i}}^{\alpha}=-\frac{\partial \mathrm{E}\left[\left\{\psi_{\mathrm{i}}\right\},\left\{\mathbf{R}_{\mathrm{i}}\right\}\right]}{\partial \mathbf{R}_{\mathrm{i}}^{\alpha}}=\mathrm{F}_{\mathrm{CP}_{\mathrm{i}}}^{\alpha} \\
\mu_{\mathrm{i}}\left|\ddot{\psi}_{\mathrm{i}}\right\rangle=-\frac{\partial \mathrm{E}\left[\left\{\psi_{\mathrm{i}}\right\},\left\{\mathbf{R}_{\mathrm{i}}\right\}\right]}{\partial\left\langle\psi_{\mathrm{i}}\right|}
\end{gathered}
$$

where $\mathrm{M}_{\mathrm{i}}$ and $\mathbf{R}_{\mathrm{i}}$ are the mass and position, respectively, of atom $\mathrm{i} ;\left|\psi_{\mathrm{i}}\right\rangle$ are the KS orbitals, which are all owed to evolve as classical degrees of freedom with inertial parameters $\mu_{i}$; and $\partial \mathrm{E}\left[\left\{\psi_{\mathrm{i}}\right\},\left\{\mathbf{R}_{\mathrm{i}}\right\}\right]$ is the $\mathrm{KS}$ energy functional evaluated for the set of ionic positions $\left\{\mathbf{R}_{\mathrm{i}}\right\}$ and the set of orbitals $\left\{\psi_{\mathrm{i}}\right\}$. The functional derivative of the KS energy is implicitly restricted to variations of $\left\{\psi_{i}\right\}$ that preserve orthonormality. Thus, the electrons are put into their ground state at a fixed set of ionic positions and with the ions moving according to the previously given equation, the electronic or bitals should adiabatically follow the motion of the ions, performing only small oscillations about the electronic ground state. The electronic orbitals will possess a "fictitious" kinetic energy, according to their motion, and, thus, a fictitious mass parameter $\mu_{\mathrm{i}}$. If $\mu_{\mathrm{i}}$ is small, then the motion of the orbitals should be fast, relative to the motion of the ions.

\subsection{Poisson-Boltzmann Theory}

The Poisson-Boltzmann (PB) theory has been used to calculate the influence of the charged groups in PEM pores on the energy barriers for proton jumps and in combination with phenomenological concepts of charge-transfer theory and the temperature dependence of the reaction rate. ${ }^{99,100}$ In the mean-field PB theory, the distribution of mobile charges (here, protons) in a pore is calculated from the boundary conditions and the arrangement of external static charges (here, sulfonate groups), neglecting both 
correlations between the mobile charges and a spatially dependent dielectric constant of the water within the pore. Because of such drastic assumptions, the density of the protons near the sulfonate groups is far too high (similar to that predicted by a simple Gouy-Chapman model; see Section 3.1.2.1). Specifying a geometric model and an arrangement of sulfonate groups (slab pores of varying width with a regular lattice of negative point charges on the pore surface $\left.{ }^{99}\right)$, proton distributions and electrostatic potential barriers for proton motion al ong the pore were calculated. Although the model did not directly yield an absolute value for proton mobility, activation energies of proton transport were estimated, as a function of water content, pore shape, and sulfonate density. A modified PB ansatz, taking into account a more realistic charge distribution for the sulfonate groups, was shown to be qualitatively consistent with MD simulations. ${ }^{27}$

\subsection{Nonequilibrium Statistical Mechanical Ion Transport Modeling}

Historically, one of the central research areas in physical chemistry has been the study of transport phenomena in electrolyte solutions. A triumph of nonequilibrium statistical mechanics has been the Debye-Hückel-Onsager-Falkenhagen theory, where ions are treated as Brownian particles in a continuum dielectric solvent interacting through CouIombic forces. Because the ions are under continuous motion, the frictional force on a given ion is proportional to its velocity. The proportionality constant is the friction coefficient and has been intensely studied, both experimentally and theoretically, for al most 100 years. ${ }^{101,102}$ The simple Stokes law, derived from hydrodynamic theory where friction increases with increasing ionic radii, is known to fail for small alkali and halide ions, and, of course, protons. ${ }^{103}$

In an effort to explain the peculiar behavior of small ions in polar solvents, two models have been proposed that attribute different phenomena to solvent response and solute or ion displacement. The first model, which is often called the solventberg model, maintains the classical view of Stokes law but with an "effective" ionic radius originating from solvation. ${ }^{104}$ Thus, with solvent molecules regarded as being bound to the ion, the radius of the solvated complex is equivalent to a Stokes radius. The other model is a dielectric friction model, which has been formulated over several decades by Born, ${ }^{105} \mathrm{Fu}$ oss, ${ }^{106}$ Boyd, ${ }^{107}$ and Zwanzig, ${ }^{108}$ with a complete theoretical framework due to Hubbard and Onsager. ${ }^{109,110}$ This model attempts to describe the dielectric response of the solvent due to perturbation by the motion of an ion. As the ion is displaced from an initial position where the solvent is polarized according to the electrostatic field due to the ion, the solvent polarization is not at equilibrium with the new position of the ion, resulting in a relaxation and consequent energy dissipation of the polarization identified as dielectric (or extra) friction. The dielectric friction is inversely related to the ionic radius. Hence, the ionsize dependence of the friction coefficient has a minimum with increasing ionic radius in both models.
The first microscopic theory for ionic friction in polar solvents was proposed by Wolynes, ${ }^{111}$ in which the ion-solvent interactions were partitioned into short-range repulsive and long-range attractive components. The friction coefficient in the Wolynes model is simplified into the following two terms:

$$
\zeta=\xi_{0}+\frac{1}{3 \mathrm{k}_{\mathrm{B}} T^{2}}\left\langle\mathrm{~F}_{\mathrm{S}}^{2}\right\rangle \tau_{\mathrm{F}}
$$

where $\zeta_{0}$ is calculated from Stokes law, $\left\langle\mathrm{F}_{\mathrm{S}}^{2}\right\rangle$ is the static mean-square fluctuation in the soft force, and $\tau_{\mathrm{F}}$ is its characteristic decay time. Hence, in the case of strong, short-ranged attractive interaction, the drag on an ion reduces to that on a solvated solventberg ion, whereas in the limit of the weak longranged attractive ion-solvent interaction case, the dielectric friction picture persists. Subsequent work of Wolynes and co-workers ${ }^{112}$ attempted to implement this model for monovalent cations (i.e., $\mathrm{Li}^{+}, \mathrm{Na}^{+}, \mathrm{K}^{+}$, $\mathrm{Cs}^{+}$) in the model solvents of water, methanol, acetonitrile, and formamide. However, their results were only marginally successful for the case of water; poor agreement with experimental results being obtained for the other solvents. Chen and Adel man ${ }^{113}$ generalized the Hubbard-Onsager theory, treating the interplay between the Stokes and dielectric friction contributions within a continuum model, but provided no insight into molecular solvent relaxation due to solute perturbations. Chong and Hirata ${ }^{114}$ used an interaction-site model and the mode coupling theory to show that the friction coefficient decomposes into hydrodynamic friction, dielectric friction, and a coupling term, according to

$$
\zeta=\zeta_{\mathrm{NN}}+\zeta_{\mathrm{ZZ}}+2 \zeta_{\mathrm{NZ}}
$$

where $\xi_{\text {NN }}$ and $\xi_{\text {ZZ }}$ denote the friction from a collective density and dielectric response of the solvent to ionic displacement respectively, and $\xi_{\mathrm{NZ}}$ the friction due to their coupling. Their work shows that both the solventberg and dielectric friction mechanisms contribute to the net friction (and thereby diffusion) of small ions and thus there is really no contradiction in the coexistence of these two models. Finally, Bagchi and co-workers ${ }^{115-117}$ have shown that the calculation of the total friction on a moving ion should involve formulation with a mode-coupling-type theory (i.e., used to compute a bare friction) and a calculation of the correlation functions. Their derivation gave the foll owing relation for the friction coefficient:

$$
\frac{1}{\zeta}=\frac{1}{\xi_{\text {bin }}+\xi_{\rho \rho}+\xi_{\text {mic,DF }}}+\frac{1}{\xi_{\text {hyd }}+\xi_{\text {hyd,DF }}}
$$

where $\xi_{\text {bin }}$ and $\xi_{\rho \rho}$ are the binary and the collective solvent number density $(\rho)$ fluctuation contributions, respectively; $\xi_{\text {mic,DF }}$ is the friction contribution originating from the coupling of the ionic field with the orientational solvent polarization mode; and $\zeta_{\text {hyd,DF }}$ and $\xi_{\text {hyd }}$ are the hydrodynamic friction contributions with and without polar contribution, respectively.

The first attempt to apply microscopic electrolyte theory to study the mobility of protons in PEMs is due to Paddison and co-workers. ${ }^{40-46}$ Because the 
foregoing discussion concerns the mobility of small ions in polar solvents, it would seem that application of this methodology to proton transport is somewhat questionable. However, because their model focuses on the coupled transport of a proton with a water molecule (i.e., an hydronium ion), there is perhaps a more substantial underpinning to the calculation of "a proton friction coefficient" in these membranes. The purpose of their nonequilibrium statistical mechanical transport model is to calculate proton selfdiffusion coefficients for hydrated PEMs from molecular and morphological information without resorting to any fitting or adjustable parameters through computation of velocity-independent friction coefficients. Because pulsed-field gradient (PFG) NMR measurements allow for the determination of membrane and hydration-specific proton self-diffusion coefficients, this kinetic model permits the identification of how molecular chemistry and membrane morphology is connected to a macroscopic quantity that ultimately determines performance of a fuel cell. Their model is based on the methodol ogy of Resibois and others, ${ }^{118}$ and, because of its surprising success in the calculation of proton self-diffusion coefficients in various PEMs, over a range of hydration levels, a brief description of the model is given here.

Similar to the work described previously, ${ }^{111-117}$ the starting point of the kinetic model is the assumption that, under linear response theory, the ensemble average force is proportional to the velocity of a hydronium ion through the friction coefficient (i.e., $\left.\left\langle\mathbf{F}_{\alpha}\right\rangle=-\xi \cdot \mathbf{v}_{\alpha}\right)$. Computation of this average force experienced by a hydronium ion as it transverses the channel, when combined with the Einstein relation, allows for the calculation of the proton self-diffusion coefficient. This pore within a PEM is assumed to possess a cylindrical geometry filled with water molecules, according to the degree of hydration of the membrane. The dissociated acidic functional groups in the pore are modeled as radially symmetric axially periodic arrays of fixed ions (i.e., point charges) and, thus, the average force experienced by the hydronium ion is calculated using the standard methods of statistical mechanics, albeit with a suitably constructed nonequilibrium distribution function. This distribution function is obtained from a formal solution of the time evolution or Liouville equation. The Liouville operator is constructed from a Hamiltonian of the system with an inertial reference frame moving at the velocity of the hydronium ion and consists of the kinetic energy of all the water molecules and the net potential energy, because of two-body interactions of the water molecules, hydronium ion, and fixed sites. The respective contributions to the potential energy of the system are due to (i) interactions of the hydronium ion with the water molecules, (ii) interaction of the hydronium ion with the arrays of the fixed sites, (iii) water-water interactions, and (iv) interactions of the water molecules with the fixed sites. Thus, the scalar friction coefficient of the hydronium ion is partitioned into four force-force correlation functions:

$$
\begin{array}{r}
\xi_{\alpha}=\frac{\beta}{3} \int_{0}^{\infty} \mathrm{dt}\left(\left\langle\mathrm{F}_{\alpha s} \mathrm{e}^{-\mathrm{i} \mathrm{L}_{0} \mathrm{t}} \mathrm{F}_{\alpha s}\right\rangle_{0}+\left\langle\mathrm{F}_{\alpha \mathrm{s}} \mathrm{e}^{-\mathrm{i} \mathrm{L}_{0} \mathrm{t}} \mathrm{F}_{p s}\right\rangle_{0}+\right. \\
\left.\left\langle\mathrm{F}_{\alpha \mathrm{p}} \mathrm{e}^{-\mathrm{i} \mathrm{L}_{0} \mathrm{t}} \mathrm{F}_{\mathrm{ps}}\right\rangle_{0}+\left\langle\mathrm{F}_{\alpha \mathrm{p}} \mathrm{e}^{-\mathrm{i} \mathrm{L}_{0} \mathrm{t}} \mathrm{F}_{\alpha s}\right\rangle_{0}\right)
\end{array}
$$

where $\beta=1 /(k T)$, and the forces $F_{\alpha s}, F_{p s}$, and $F_{\alpha p}$ are between the hydronium ion and the water molecules, the fixed sites and the water molecules, and the hydronium ion and the fixed sites, respectively. Only the latter threeterms are explicitly evaluated: their sum is taken to be a correction, $\xi^{(c)}$, to a "zeroth order" friction coefficient. Because this first force-force correlation function involves only the force that the water exerts on the hydronium ion $\left(F_{\alpha s}\right)$, it is taken to be either the friction coefficient of a hydronium ion in bulk water calculated with the Stokes relation (this is typically the case for minimally or only partially hydrated membranes), or the friction coefficient of a proton in bulk water derived from experimental diffusion measurements (used for fully hydrated membranes). Structure diffusion of the proton is significant in bulk water (see Section 3.1.1.1.1); thus, their model does account for this contribution to the mobility of the proton in membrane pores through the first term in eq 20 , i.e., $F_{\alpha s}$. The choice of the numerical value of this zeroth-order term is not arbitrary but is dependent on the characteristics (i.e., structure) of the water in the pore; and the latter is assessed using the dielectric saturation model described in the following section.

\subsection{Dielectric Saturation}

In a typical hydrated PEM, the rigidity of the backbone and the crystallinity of the polymer confine the water (to regions of only nanometers); and with the significant density and distribution of the pendant anionic groups give structural ordering to the water in the membrane. Both experimental ${ }^{119-122}$ and MD simulations ${ }^{123-126}$ have revealed that water confined in systems such as reverse micelles and biological pores possesses a decreased polarity and rate of relaxation, and an increased degree of spatial and orientational order, when compared to bulk water. As such, assumptions of a constant dielectric constant for the water in the pore of a PEM (either that of bulk water or some other values) is clearly incorrect. The water near the polymer backbone and fixed anionic groups is more constrainted and ordered than the water located in the center of the pores. This phenomenon resembles that observed for an electrolyte near a charged electrode for which various models have been formulated, including Helmholtz, Gouy-Chapman, Stern, and Grahame (see Section 3.1.2.1).

Structure diffusion (i.e., the Grotthuss mechanism) of protons in bulk water requires formation and cleavage of hydrogen bonds of water molecules in the second hydration shell of the hydrated proton (see Section 3.1); therefore, any constraint to the dynamics of the water molecules will decrease the mobility of the protons. Thus, knowl edge of the state or nature of the water in the membrane is critical to understanding the mechanisms of proton transfer and transport in PEMs. 
Hence, the recent work of Paul and Paddison, ${ }^{47-51}$ which was alluded to previously, has sought to describe the spatially dependent dielectric constant of the water in PEMs. Their work follows in the spirit of the much earlier pioneering work of Booth ${ }^{127}$ and more recent application of Bontha and Pintauro. ${ }^{128}$ They assume that the field-dependent permittivity of the water, $\epsilon(E)$, may be expressed as the sum of two terms, according to

$$
\epsilon(E)=n^{2}+\frac{4 \pi P(E)}{\epsilon_{0} E}
$$

where $\mathrm{n}$ is the refractive index and $\mathbf{E}$ and $\mathbf{P}$ are the magnitudes of the electric field and polarization, respectively. The polarization is computed from a realization that it is a functional derivative of the Helmholtz energy, A, i.e.,

$$
\mathrm{P}\left(\mathrm{r}, \mathrm{E}, \mathrm{E}_{\mathrm{e}}\right)=-\frac{\delta \mathrm{A}\left(\mathrm{E}_{\mathrm{e}} \mathrm{E}_{\mathrm{e}}\right)}{\delta \mathrm{E}_{\mathrm{e}}(\mathbf{r})}
$$

where the dependence of both the polarization and energy on the electrostatic field due to the fixed anionic groups $(E)$ and an external "probing" el ectric field $\left(E_{e}\right)$ are explicitly declared. One of the important implications of eq 22 is the inclusion of the electrostatic field as part of the total energy (Hamiltonian) of the system, which removes the nonphysical results of divergence in the dielectric constant.

\section{Transport Mechanisms}

\subsection{Proton Conduction Mechanisms}

Despite the variety of proton-conducting separator materials, the inherent protonic charge carriers (protonic defects) are solvated by very few types of species. These are essentially water (e.g., in hydrated acidic polymers), oxo-acid anions (e.g., in $\mathrm{CsHSO}_{4}$ ) or oxo-acids such as phosphoric acid (e.g., in adducts of basic polymers with phosphoric acid), heterocycles (e.g., intercalated into aci dic polymers or immobilized via flexible spacers), or oxide ions (forming a hydroxide on the oxygen site of an oxide lattice). These species participate in the formation of protonic charge carriers and the proton conduction mechanism. In a few cases, they are also the protogenic group: i.e., they generate protonic charge carriers by self-dissociation (e.g., in the case of phosphoric acid and to some extent also in heterocycles such as imidazole); in other cases, the protonic charge carriers must be generated extrinsically, by doping with a Brønsted acid or base (i.e., in water containing systems and oxides).

A common and important characteristic of all these species is their involvement in hydrogen bonding. The structural and dynamical nature of this interaction seems to be the key to understanding long-range proton transport in these environments. Strong hydrogen bonding is frequently considered to be a precursor of proton-transfer reactions; ${ }^{129}$ however, long-range proton transport also requires rapid bond breaking and forming processes, which is only ex- pected to occur in weakly hydrogen bonded systems. Dynamical bond-length variations have long been recognized to be integral for the hydrogen bonding in proton-conducting systems; ${ }^{130}$ however, it is only recently that the appearance of such "dynamical hydrogen bonding" has been explained through a consideration of the chemical interactions of the systems (including hydrogen bonding) in a wide range of configuration space.131 The interplay of hydrogen bonds with other intermolecular forces is generally examined in refs 132-136.

Proton-conduction mechanisms that occur in the aforementioned proton solvents, when present as a homogeneous phase and as a component of heterogeneous fuel cell separator materials, are described more specifically in the following two sections.

\subsubsection{Homogeneous Media}

3.1.1.1. Water and Aqueous Solutions. 3.1.1.1.1. Mobility of Excess Protons. The unusually high mobility (equivalent conductivity) of protons in water and aqueous solutions (under ambient conditions, $\sim 9$ times higher than that for $\mathrm{Li}^{+}$and $\sim 5$ times higher than that for $\mathrm{K}^{+}$) has been investigated since the early days of physical chemistry, and the different concepts and approaches have been summarized several times. ${ }^{34}$ The essential features of the present view dates back to the work of Eigen and De Maeyer. ${ }^{137,138}$ They demonstrated that "structure diffusion", i.e., the "diffusion" of the structure (hydrogen-bond pattern) in which the excess proton is "tunneling" back and forth, is the rate-limiting step. Ever since, there has been some controversy as to whether the region containing the excess proton may be described as a hydrated hydronium ion (i.e., $\mathrm{H}_{9} \mathrm{O}_{4}{ }^{+}$, later termed the Eigen ion) or a smaller dimer sharing the excess proton (i.e., $\mathrm{H}_{5} \mathrm{O}_{2}{ }^{+}$, the Zundel ion ${ }^{139}$ ). This debate was largely resolved by the mechanistic details obtained from CPMD simulations (see Section 2.2.3) by Tuckerman et al. ${ }^{34,35}$ and the interpretation of NMR data by Agmon. ${ }^{140}$ I ndependently, they determined a mechanism for the diffusion of excess protons in water, which is illustrated in Figure 1.

The region with an single excess proton within the hydrogen-bond network (protonic defect) corresponds to either a Zundel ion or an Eigen ion. Interestingly, the center of the region of excess charge coincides with the center of symmetry of the hydrogen-bond pattern, ${ }^{131}$ i.e., apart from the bonds with the common shared proton, each water molecule of the Zundel ion acts as a proton donor through two hydrogen bonds, and each of the three outer water molecules of the Eigen ion acts as a proton donor in two hydrogen bonds and as an acceptor for the hydronium ion and an additional water molecule (see Figure 1). Changes to these hydrogen-bond patterns through hydrogen-bond breaking and forming processes displaces the center of symmetry in space and, therefore, also the center of the region of excess charge. In this way, a Zundel ion is converted to an Eigen ion, which then transfers into one of three possible Zundel ions (see Figure 1). This type of mechanism may still be termed "structure diffusion" 


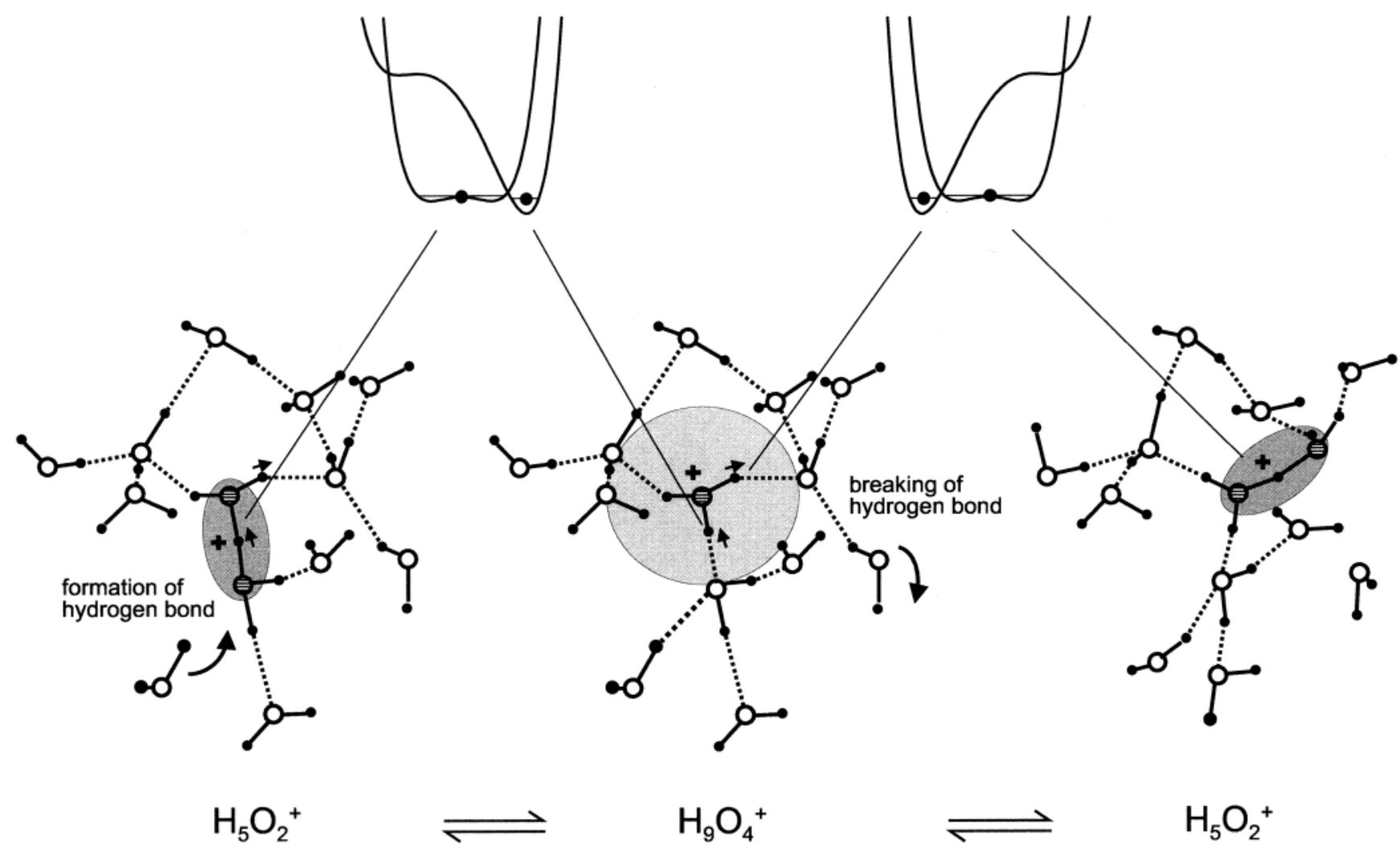

(Zundel-ion)

(Eigen-ion)

(Zundel-ion)

Figure 1. Proton conduction in water. The protonic defect follows the center of symmetry of the hydrogen-bond pattern, which "diffuses" by hydrogen-bond breaking and forming processes; therefore, the mechanism is frequently termed "structure diffusion". Note that the hydrogen bonds in the region of protonic excess charge are contracted, and the hydrogen-bond breaking and forming processes occur in the outer portion of the complexes (see text). Inserted potentials correspond to nonadiabatic transfer of the central proton in the three configurations (atomic coordinates taken from refs 33 and 34 ).

(as suggested by Eigen), because the protonic charge follows a propagating hydrogen-bond arrangement or structure.

The sum of all proton displacements involved in the hydrogen-bond breaking and forming processes and the proton displacements within the hydrogen bonds of the Zundel and Eigen ions then corresponds to the net displacement of one unit charge by just a little more than the separation of the two protons in a water molecule (i.e., 200 pm). Although there are no individual, exceptionally fast protons, even on a short time scale, the fast diffusion of protonic defects leads to a slight increase in the physical diffusion of all protons in the system. This is indeed observed for aqueous solutions of hydrochloric acid, for which mean proton diffusion coefficients were observed to be up to $5 \%$ higher than the diffusion coefficient of oxygen, as measured by ${ }^{1} \mathrm{H}$ and ${ }^{17} \mathrm{O}$ PFG NMR, ${ }^{141}$ reflecting the slightly attenuated correlation of proton and oxygen diffusion in acidic media.

Another interesting feature of this mechanism is that the hydrogen-bond breaking and forming (hydrogen-bond dynamics) and the translocation of protons within the hydrogen bonds occur in different parts of the hydrogen bond network, al beit in a highly concerted fashion. This is the most thermodynamically favorable transport path, because the hydrogen bonds in the center of the two charged complexes are contracted to such an extent that this allows an almost barrierless proton translocation while the hydrogen-bond breaking and forming processes occur in the weakly bonded outer parts of the complexes. This contraction of the center of the complex is probably a direct consequence of the lower coordination of the involved species ( 3 instead of $\sim 4$ ). The activation enthalpy of the overall transport process is dominated by the hydrogen-bond breaking and forming, which also explains the strong correlation of the proton transport rate and the dielectric relaxation. ${ }^{142}$ The Zundel and Eigen complexes are just limiting configurations, and the simulations indeed produce configurations that can hardly be ascribed to one or the other. ${ }^{36}$ Although simulations find comparable probabilities for the occurrence of both type of complexes, ${ }^{34,35}$ there is experimental evidence for a slight stabilization of the Eigen complex, compared to the Zundel complex, by $\sim 2.4 \mathrm{~kJ} / \mathrm{mol}(25$ $\mathrm{meV}) .{ }^{143} \mathrm{H}$ owever, these differences are marginal and do not change the principal features of the mechanism.

One such feature is the potential energy surface for proton transfer in the contracted hydrogen bonds. The time-averaged potential surfaces are almost symmetrical (especially for the Zundel ion) without significant barriers, i.e., the proton is located near the center of the bond. Whether its location is offcenter at any time instance is mainly dependent on the surrounding hydrogen-bond pattern, and it is the 
change of this pattern that alters the shape (and asymmetry) of this potential and, therefore, the position of the proton within the hydrogen bond (see Figure 1, top). In other words, the proton is transferred almost adiabatically, with respect to the solvent coordinate. ${ }^{1}$ This has important consequences on the mechanism when static asymmetric contributions are introduced, i.e., by chemical interactions or the presence of ionic charges (see below). The very low barriers are also the reason for the fact that the mechanism can be well described classically, with respect to the motion of the nuclei (especially the proton); in particular, proton tunneling has only a minor effect on the rate of transfer. Nevertheless, the protonic defect (region of protonic excess charge) may become del ocalized through several hydrogen bonds, because of quantum fluctuations. ${ }^{36,85}$

The mechanism also provides insight into the question as to what extent proton transfer in water is a cooperative phenomenon. In many physical chemistry textbooks, one still finds diagrams showing the concerted transfer of protons within extended hydrogen-bonded water chains (the Grotthuss mechanism) to explain the unusually high equivalent conductivity of protons in this environment. However, the creation of the corresponding dipolar moment in an unrelaxed high dielectric constant environment costs far too much energy to be consistent with a very fast process with low activation energy. ${ }^{1,144}$ As anticipated in ref 1 , the propagation mechanism of a protonic defect in a low-dimensional water structure surrounded by a low dielectric environment is obviously between "concerted" and "step-wise"; 145,146 however, in bulk water, the cooperation is restricted to the dynamics of protons in neighboring hydrogen bonds (also see Figure 1).

Onealso should keep in mind that water is a liquid with a high self-diffusion coefficient (i.e., $\mathrm{D}_{\mathrm{H}_{2} \mathrm{O}}=2.25$ $\times 10^{-5} \mathrm{~cm}^{2} / \mathrm{s}$ at room temperature) and that the diffusion of protonated water molecules makes some contribution to the total proton conductivity (vehicle mechanism ${ }^{147}$ ). This is 22\% when assuming that the diffusion coefficients of $\mathrm{H}_{2} \mathrm{O}$ and $\mathrm{H}_{3} \mathrm{O}^{+}$(or $\mathrm{H}_{5} \mathrm{O}_{2}{ }^{+}$) are identical. However, as suggested by Agmon, ${ }^{148}$ the diffusion of $\mathrm{H}_{3} \mathrm{O}^{+}$may be retarded, because of the strong hydrogen bonding in the first hydration shell.

Of course, the relative contributions of "structure diffusion" and "vehicular diffusion" are dependent on temperature, pressure, and the concentrations and types of ions present. With increasing temperature, structure diffusion is attenuated and with increasing pressure the contribution of structure diffusion increases until it reaches a maximum at $\sim 0.6 \mathrm{GPa}(6$ kbar). ${ }^{1}$ Especially relevant for the later discussion of proton transport in hydrated acidic membranes is the observation that structure diffusion strongly decreases as the acid concentration increases (see Figure 2 and ref 141), which is probably due to changes in the hydrogen-bond pattern (there are progressively more proton donors than corresponding proton acceptor "sites") and a consequence of the biasing of the hydrogen bonds in the electrostatic field of the ions suppressing the proton-transfer mechanism illustrated in Figure 1.

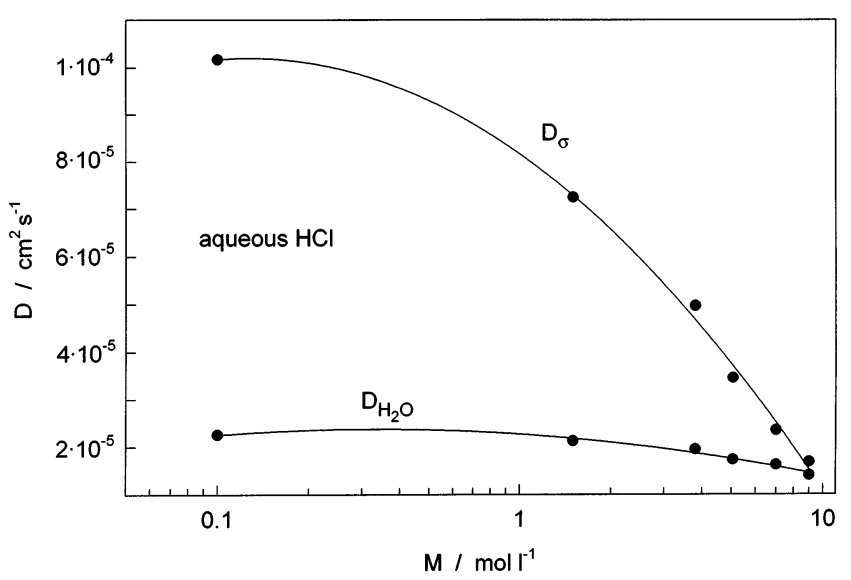

Figure 2. Conductivity diffusion coefficient (mobility) of protons and water self-diffusion coefficient of aqueous solutions of hydrochloric acid $(\mathrm{HCl})$, as a function of acid concentration (molarity, M) (data are taken from ref 141).

3.1.1.1.2. Mobility of Defect Protons. Because basic aqueous solutions also have some relevance for fuel cell applications (e.g., in alkaline fuel cells (AFCs)), the transport mechanism of defect protons $\left(\mathrm{OH}^{-}\right)$is also reviewed here. Although intuition might lead one to assume that the mechanism is reminiscent of that of an excess proton, recent CPMD simulations suggest that this may not be the case. ${ }^{149}$ As opposed to Zundel and Eigen complexes, in which the central species are only 3-fold coordinated (under-coordinated, with respect to water in pure water, which is probably the reason for the bond contraction in these complexes; see above) on the average, the hydroxide ion is observed to be coordinated by $\sim 4.5$ water molecules in an almost-planar configuration with the $\mathrm{OH}$ proton pointing out of the plane. This is considered to be a true quantum effect and contradicts the common understanding of a 3-fold coordination. ${ }^{150}$ This "hyper-coordination" is suggested to prevent proton transfer from a $\mathrm{H}_{2} \mathrm{O}$ to the $\mathrm{OH}^{-}$, because this would produce an unfavorable $\mathrm{H}-\mathrm{O}-\mathrm{H}$ bond angle of $90^{\circ}$. The proton transfer only occurs when the $\mathrm{OH}^{-}$ coordination is reduced to 3 by breaking one of the 4 hydrogen bonds within the plane and some rearrangement of the remaining bonds occurs, which allows the direct formation of a water molecule with a tetrahedral geometry. Surprisingly, the groundstate coordination of the most favorable configurations around excess protons seem to be similar to the coordination of the transition state for the transport of defect protons. Note, that hyper-coordination of the $\mathrm{OH}^{-}$is still a matter of controversial debate. The statistical mechanical quasi-chemical theory of solutions suggests that tricoordinated $\mathrm{OH}^{-}$is the predominant species in the aqueous phase under standard conditions. ${ }^{151,152}$ This finding seems to be in agreement with recent spectroscopic studies on hydroxide water clusters, and it is consistent with the traditional view of $\mathrm{OH}^{-}$coordination.

It should also be mentioned that $\mathrm{OH}^{-}$"hypercoordination" is not observed in concentrated solutions of $\mathrm{NaOH}$ and $\mathrm{KOH} .{ }^{153}$ In contrast to acidic solutions, where structure diffusion is suppressed with increasing concentration (see above and Figure 2) the transference number of $\mathrm{OH}^{-}$(e.g., in aqueous 
$\mathrm{KOH}$ solutions) remains surprisingly high ( $\sim 0.74)$ for concentrations up to $\sim 3 \mathrm{M}$.

In pure water, excess protons $\left(\mathrm{H}_{3} \mathrm{O}^{+}, \mathrm{H}_{5} \mathrm{O}_{2}{ }^{+}\right)$and defect protons $\left(\mathrm{OH}^{-}\right)$are present at identical concentrations; however, because of their low concentration ( $10^{-7} \mathrm{M}$ under ambient conditions), the diffusion of these defects may be considered to be quasi-independent. Only in small water clusters do zwitterions show a remarkable stability. ${ }^{154}$

3.1.1.2. Phosphoric Acid. Although the proton conduction mechanism in phosphoric acid has not been investigated to the same extent, it is evident that the principal features exhibit similarities, along with important differences.

Above its melting point of $\mathrm{T}_{\mathrm{m}}=42{ }^{\circ} \mathrm{C}$, neat phosphoric acid $\left(\mathrm{H}_{3} \mathrm{PO}_{4}\right)$ is a highly viscous liquid with extended intermolecular hydrogen bonding. However, in contrast to the situation in water, there are more possible donor than acceptor sites and the amphoteric character is significantly more pronounced: phosphoric acid may act as both a Brønsted acid and base. In terms of equilibrium constants, both $\mathrm{K}_{\mathrm{a}}$ and $\mathrm{K}_{\mathrm{b}}$ are reasonably high ( $\mathrm{K}_{\mathrm{a}}$ of the conjugate base is (ow). Consequently, phosphoric acid shows a very high degree of self-dissociation (autoprotolysis) of $\sim 7.4 \%, 155$ along with some condensation, with $\mathrm{H}_{2} \mathrm{PO}_{4}^{-}, \mathrm{H}_{4} \mathrm{PO}_{4}{ }^{+}, \mathrm{H}_{3} \mathrm{O}^{+}$, and $\mathrm{H}_{2} \mathrm{P}_{2} \mathrm{O}_{7}{ }^{2-}$ being the main dissociation products. Because of their high concentration, the separation of the overall conductivity into charge carrier concentration and mobility terms is problematic. Nevertheless, the proton mobility has been calculated from total conductivities by the Nernst-Einstein equation by taking concentrations from ref 155; and the values have been observed to be almost 2 orders of magnitude higher than the values for the diffusion coefficient of the diverse phosphate species obtained directly by ${ }^{31} \mathrm{P}$ PFG NMR ${ }^{156}$ and estimated from viscosity measurements via the Stokes-Einstein relation.

Pure phosphoric acid is a liquid with a low diffusion coefficient of phosphate species but an extremely high proton mobility, which must involve proton transfer between phosphate species and some structural rearrangements. The contribution to the total conductivity is 98\%; i.e., phosphoric acid is an almost-ideal proton conductor. The total conductivity at the melting point $\left(\mathrm{T}=42{ }^{\circ} \mathrm{C}\right)$ is $7.7 \times 10^{-2} \mathrm{~S} / \mathrm{cm}$, with an estimated proton mobility of $2 \times 10^{-5} \mathrm{~cm}^{2} / \mathrm{s}$. ${ }^{156}$ This extremely high proton mobility has also been indirectly determined with ${ }^{1} \mathrm{H}$ PFG NMR and was observed to be even higher (by a factor of 1.5-2.3). This has been explained by the correlated motion of the oppositely charged defects $\left(\mathrm{H}_{2} \mathrm{PO}_{4}^{-}, \mathrm{H}_{4} \mathrm{PO}_{4}{ }^{+}\right)$ when they are close to one another (i.e., the case just after their formation (by dissociation of $\mathrm{H}_{3} \mathrm{PO}_{4}$ ) and before their neutralization). Correlation effects are actually quite common in proton conductors with high concentrations of charge carriers and they are even more pronounced in other systems (see Section 3.1.1.3 on heterocycles and proton transport in alkaline-metal hydroxides ${ }^{157,158}$ ).

Molecular details of the structure diffusion mechanism with the hydrogen-bond breaking and forming and the proton transfer between the different phos- phate species (essentially $\mathrm{H}_{2} \mathrm{PO}_{4}^{-}, \mathrm{H}_{3} \mathrm{PO}_{4}, \mathrm{H}_{4} \mathrm{PO}_{4}^{+}$) have not been investigated yet; however, the high degree of self-dissociation suggests that the protontransfer events are even less-correlated than in water (the system is more tolerant toward protonic charge density fluctuations). The transfer events are probably almost barrierless, as indicated by negligible $\mathrm{H} / \mathrm{D}$ effects of the diffusion coefficients in mixtures of $\mathrm{H}_{3} \mathrm{PO}_{4}$ and $\mathrm{D}_{3} \mathrm{PO}_{4}$. ${ }^{130}$

The principal proton transport mechanism remains essentially the same with the addition of some water, with a conductivity increase up to $0.25 \mathrm{~S} / \mathrm{cm}$ under ambient conditions. A ${ }^{1} \mathrm{H}$ and ${ }^{31} \mathrm{P}$ PFG NMR study also showed that an $85 \mathrm{wt} \%$ phosphoric acid system was an almost ideal proton conductor, with $98 \%$ of the conductivity originating from the structure diffusion of protons. ${ }^{159}$ The combination of high intrinsic charge carrier concentration and mobility renders the possibility of very high conductivities in these systems. In particular, there is no perturbation from extrinsic doping, i.e., there is no suppression of structure diffusion, despite the high concentration of protonic charge carriers. On the other hand, attempts to increase the conductivity of phosphoric acid-based systems by doping have expectedly failed. ${ }^{160}$

3.1.1.3. I midazole. Historically, the interest in hydrogen bonding and proton conductivity in heterocycles has its roots in speculations about the participation of hydrogen bonds in energy and charge transfer in biological systems; ${ }^{161}$ specifically, concerning the participation of $\mathrm{NH} \cdots \mathrm{N}$ bonds between the imidazole groups of histidine in proton transport in transmembrane proteins. ${ }^{162}$ Even Zundel has worked in the field, ${ }^{163}$ and it is not surprising that his view of the proton dynamics in imidazole is closely related to that of water. He suggested a high polarizability of the protons within intermolecular hydrogen bonds and, as a consequence, a very strong coupling between hydrogen bonds, as indicated by the intense IR continuum in the NH stretching regime. Surprisingly, he did not suggest the existence of any complex similar to the Zundel complex in water ${ }^{163}$ (see Section 3.1.1.1), whereas Riehl164 had already suggested "defect protons" or "proton hol es" as requirements to maintain a current in solid imidazole. Early conductivity measurements had their focus on crystalline monoclinic imidazole, which has a structural hydrogen bond length of 281 pm. ${ }^{165}$ The measured conductivities were typically low (i.e., $\sim 10^{-8} \mathrm{~S} / \mathrm{cm}$ ) with very poor reproducibility. ${ }_{161,166,167}$ Later tracer experiments ${ }^{168}$ and a ${ }^{15}$ N N M R study ${ }^{169}$ raised doubts about the existence of proton conductivity in pure crystalline imidazole.

However, the conductivity of liquid imidazole is several orders of magnitude higher $\left(\sim 10^{-3} \mathrm{~S} / \mathrm{cm}\right.$ at the melting point of $\left.\mathrm{T}_{\mathrm{m}}=90^{\circ} \mathrm{C}\right) ; 161$ however, its mechanism was investigated much later. It was the search for chemical environments that were different from water in fuel cell membranes that brought heterocycles back into focus. The potential proton donor and acceptor functions (amphoteric character), the low barrier hydrogen bonding between the highly polarizable $\mathrm{N}$ atoms, and the size and shape of the molecule were reasons that Kreuer et al. ${ }^{170}$ started 
to investigate the usefulness of heterocycles as proton solvents in separator materials for fuel cells. This work also comprises the study of the transport properties of neat and acidified liquid imidazole, pyrazole, and, later, benzimidazole. ${ }^{171}$ An important finding was that the transport coefficients (i.e., mobility of protonic charge carriers and molecular diffusion coefficients) are similar to those of water at a given temperature, relative to the melting point. This is particularly true for their ratio: i.e., the proton mobility is a factor of $\sim 4.5$ higher than the molecular diffusion coefficient at the melting point of imidazole. ${ }^{170}$ This is a direct indication of fast intermolecular proton transfer and the possibility of structure diffusion in this environment. Subsequently, details were revealed by a CPMD simulation. ${ }^{37}$ In contrast to earlier suggestions of concerted proton transfer in extended chains of hydrogen bonds ${ }^{161,172}$ (analogous to the proton conduction mechanism in water presented in most textbooks at that time $^{144}$ ), a structure diffusion mechanism similar to that for water (Figure 1 ) was observed. The region containing the excess proton (intentionally introduced) is an imidazole with both nitrogens protonated and acting as proton donors toward the two next nearest imidazoles in a configuration I $\mathrm{mi} \cdots . \mathrm{miH}^{+} . . .1 \mathrm{mi}$ with hydrogen bonds ( $\left.273 \mathrm{pm}\right)$ slightly contracted, compared to the average bond length of the system, but still longer than the bonds in the isolated complex (in the gas phase). ${ }^{173}$ The position of the protons within these hydrogen bonds is dependent mainly on the hydrogen bonding between the nearest and next-nearest solvating imidazoles (Figure 3). The hydrogen-bonded structure in imidazole is observed to be chainlike (i.e., low dimensional), with two possible orientations of the hydrogenbond polarization within segments which are separated by imidazoles with their protonated nitrogen directed out of the chain. This may even form a "cross-linking" hydrogen bond with a nonprotonated nitrogen of a neighboring strand of imidazole. The simulation data revealed the existence of imidazole molecules close to the protonic defect in hydrogenbond patterns, which rapidly change by bond breaking and forming processes. Similar to water, this shifts the excess proton within the region and may even lead to complete proton transfer, as displayed in Figure 3. There is no indication of the stabilization of a symmetrical complex $(\mathrm{Imi} \cdots \mathrm{H} \cdots \mid \mathrm{mi})^{+}$: there always seems to be some remaining barrier in the hydrogen bonds, with the proton being on one side or the other.

As for the CPMD simulation of water, the simulated configuration is artificial, because there is no counter charge compensating for the charge of the excess proton. This is necessary, methodologically, because self-dissociation is unlikely to occur within the simulation box used (i.e., eight imidazole molecules with a single excess proton) and the accessible simulation time ( 10 ps). The self-dissociation constants for heterocycles (in particular, imidazole) are actually much higher than for water, but degrees of self-dissociation (concentration of protonic charge carriers) of $\sim 10^{-3}$ are still $\sim 2$ orders of magnitude
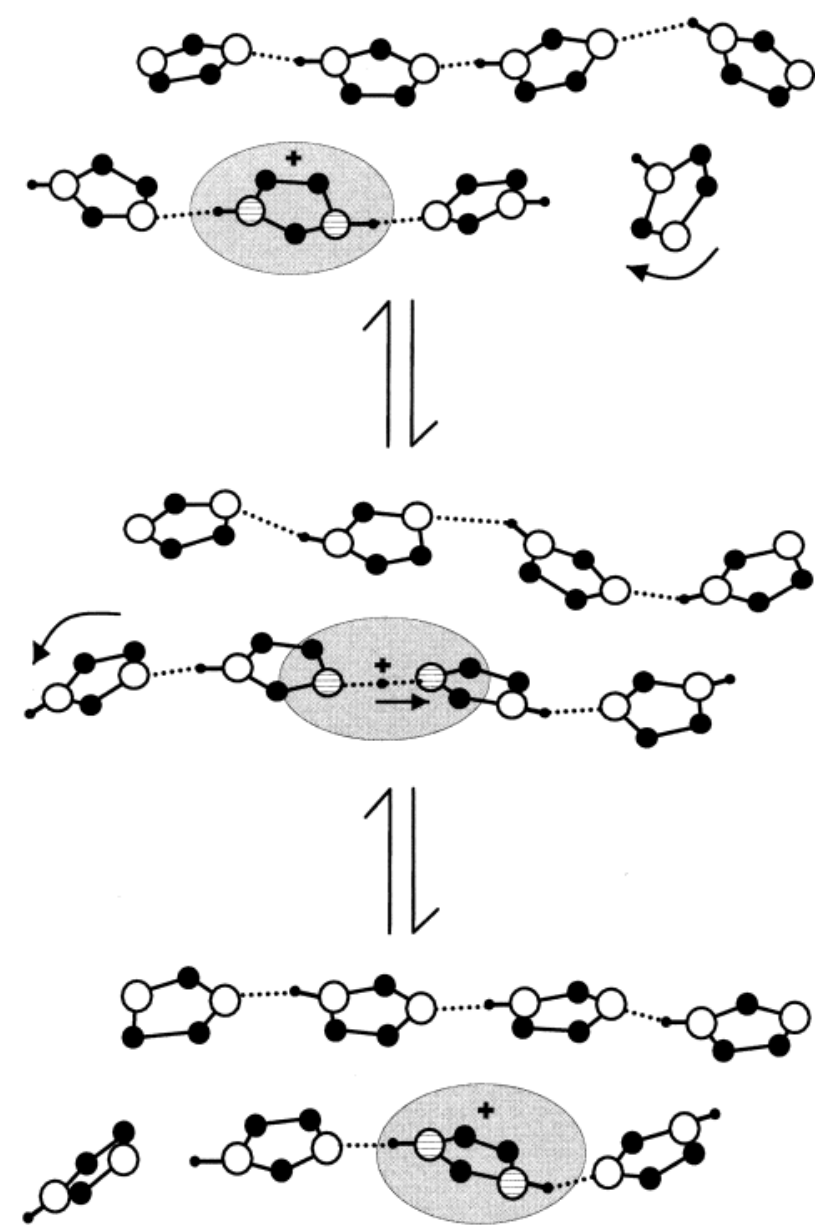

Figure 3. Proton conduction mechanism in liquid imidazole, as revealed by a Car-Parrinello molecular dynamics (CPMD) simulation. ${ }^{37}$ Note the similarities with the proton conduction mechanism in water (see Figure 1).

lower than that for phosphoric acid (see Section 3.1.1.2). Site-selective proton diffusion coefficients (obtained by ${ }^{1} \mathrm{H}$ PFG NMR of different imidazolebased systems) show surprisingly high diffusion coefficients for the protons involved in hydrogen bonding between the heteroatoms (nitrogen). ${ }^{174} \mathrm{De}-$ pending on the system, they are significantly higher than calculated from the measured conductivities corresponding to Haven ratios $\left(\sigma_{\mathrm{D}} / \sigma\right)$ of $3-15$. This indicates some correlation in the diffusion of the proton, which may be due to the presence of a counter charge neglected in the simulation.

In pure imidazole, regions containing excess protons must be charge-compensated by proton-deficient regions with electrostatic attraction between these regions (defects) that is dependent on their mutual separation distance and the diel ectric constant of the medium. Under thermodynamic equilibrium, such defects are steadily formed and neutralized. Formally, the creation of a protonic defect pair is initiated by a proton transfer from one imidazole to another, with the subsequent separation of the two charged species with a diffusion mechanism as described previously (also see Figure 3). However, this transfer is contrary to the electrostatic field of the counter charge, favoring a reversal of the dissociation process. However, because the two protons of the positively charged imidazolium $\left(\mathrm{ImiH}^{+}\right)$are 

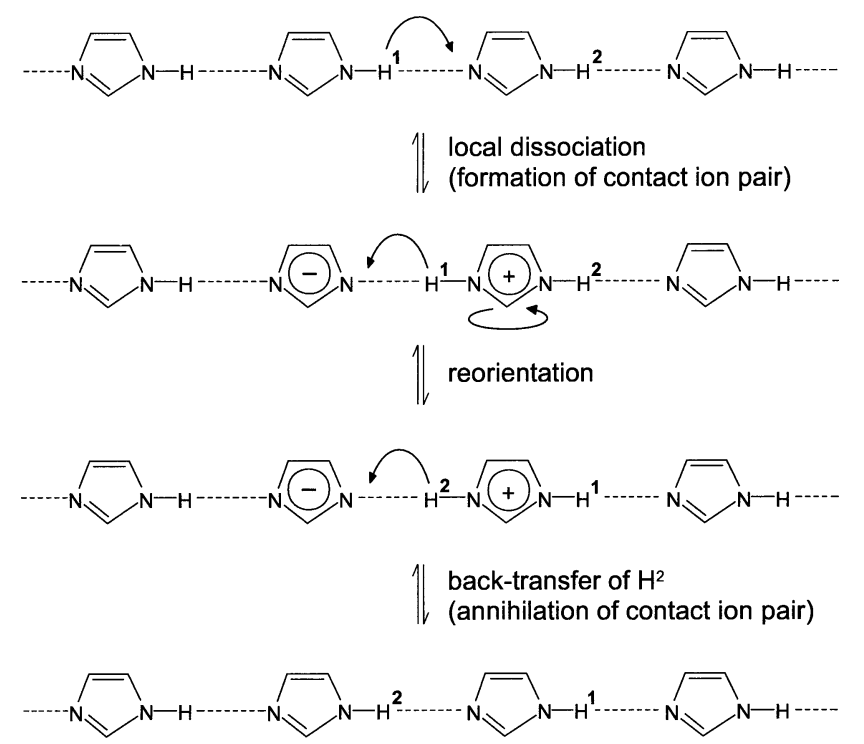

Figure 4. Schematic illustration of correlated proton transfers in pure liquid imidazole leading to proton diffusion but not proton conductivity (see text).

equivalent, there is a $50 \%$ chance that another proton is transferred back, provided that the orientational coherence between the dissociating molecules is completely lost. If the same proton is transferred back, the transient formation and neutralization of an ion pair contributes neither to the proton diffusion nor to the proton conductivity. However, if the other proton is transferred back, the protons interchange their positions in the hydrogen bond network, which generates diffusion but no conductivity since the transient charge separation is completely reversed. As illustrated for the most simple mechanism of this type in Figure 4, the sum of all proton translocation vectors form a closed trajectory reminiscent of cyclic intermolecular proton-transfer reactions known to occur in certain organic pyrazole-containing complexes ${ }^{175}$ and proton diffusion in hydroxides. ${ }^{157,158}$

Presently, there is no direct proof for such a mechanism in pure imidazole (e.g., by ${ }^{15} \mathrm{~N} \mathrm{NMR}$ ); however, the observation that the ratio of the proton diffusion and conduction rates virtually coincide with the Boltzmann factor (i.e., $\exp \left(-\mathrm{E}_{\Phi}(\epsilon) /(\mathrm{kT})\right)$, where $E_{\Phi}$ is the electrostatic separation energy of two unit charges in a continuum of dielectric constant $\epsilon$ ) is a strong indication.

This observation is also indicative of the importance of a high dielectric constant to enable the formation of protonic charge carriers and to allow their mobility being uncorrelated with their conjugated base. Of course, this is also true for the separation of protonic charge carriers from extrinsic dopants such as acids. In addition to the perturbation of the hydrogen bond network (as observed in aqueous solutions), electrostatic effects may also explain the saturation of proton conductivity with increasing acidity of the heterocyclic systems. ${ }^{171}$ This effect is even more pronounced in polymeric systems with immobilized protogenic groups and will be discussed in Section 3.1.2.3.

3.1.1.4. Simple Cubic Perovskites. Since the work of Stotz and Wagner ${ }^{176}$ in 1966, the existence of protonic defects in wide-band-gap oxides at high temperatures is well-established; and the initial notation $\mathrm{OH}_{\mathrm{O}}^{\circ}\left(\mathrm{H}_{\mathrm{i}}^{\circ}\right)$, i.e., the assumption that the defect is a hydroxideion residing on an oxide ion site carrying a positive relative charge, is still valid. More than a decade later, the systematic investigation of acceptor-doped oxides such as $\mathrm{LaAlO}_{3}, \mathrm{LaYO}_{3}$, or $\mathrm{SrZrO}_{3}$, which had already been known for their moderate oxide ion conductivity, provided experimental evidence that these materials may be proton conductors in hydrogen-containing environments. ${ }^{177}$ The observed conductivities in these materials were quite low; however, later related compounds based on $\mathrm{SrCeO}_{3}{ }^{178}$ and $\mathrm{BaCeO}_{3}{ }^{179}$ with high proton conductivities have been discovered and even tested in different types of el ectrochemical cells, including fuel cells, ${ }^{180-185}$ but the lack of stability under fuel-cell operating conditions remained an unsolved problem. Almost another two decades passed before oxides were synthesized that combined high proton conductivity with high thermodynamic stability. ${ }^{186-188}$ This brought these materials closer to a realistic alternative for fuel-cell applications and, hence, their transport properties are reviewed here.

The highest conductivities are observed in oxides with perovskite-type structures $\left(\mathrm{ABO}_{3}\right)$ with cubic or slightly reduced symmetry. ${ }^{188}$ Protonic defects are formed by the dissociative absorption of water, which requires the presence of oxide ion vacancies $V_{0} \cdot$. The latter may be formed intrinsically by varying the ratio of main constituents or extrinsically to compensate for an acceptor dopant (lower-valent cation). To form protonic defects, water from the gas-phase dissociates into a hydroxide ion and a proton, with the hydroxide ion filling an oxide ion vacancy and the proton forming a covalent bond with a lattice oxygen. In Kröger-Vink notation, this reaction is written

$$
\mathrm{H}_{2} \mathrm{O}+\mathrm{V}_{\mathrm{O}}^{\bullet}+\mathrm{O}_{\mathrm{O}}^{\times} \rightleftharpoons 2 \mathrm{OH}_{\mathrm{O}}^{\circ}
$$

by which two hydroxide ions substituting for oxide ions, i.e., two positively charged protonic defects $\left(\mathrm{OH}_{\mathrm{o}}^{\circ}\right)$, are formed. The crystallographic (time-averaged) structure of such a defect is shown in Figure 5 . There are eight orientations of the hydroxide ion stabilized by a hydrogen bond interaction with the eight next-nearest oxygen neighbors in the cubic perovskite structure. As opposed to the cases discussed previously, for which hydrogen bonding is the dominant intermolecular interaction, here, hydrogen bonding is restricted to the defect region. Within this region, hydrogen bonding interferes with other chemical interactions and, together with these, determines the structure and dynamics of the defective region.

As shown by DFTB and CPMD simulations, the principal features of the transport mechanism are rotational diffusion of the protonic defect and proton transfer toward a neighboring oxide ion. ${ }^{189-191}$ That is, only the proton shows long-range diffusion, whereas the oxygens reside in their crystallographic positions. Both experiments ${ }^{192-194}$ and quantum-MD simulations, ${ }^{189,190,195,196}$ have revealed that rotational diffusion is fast with a low activation barrier. This suggests that the proton-transfer reaction is the rate- 


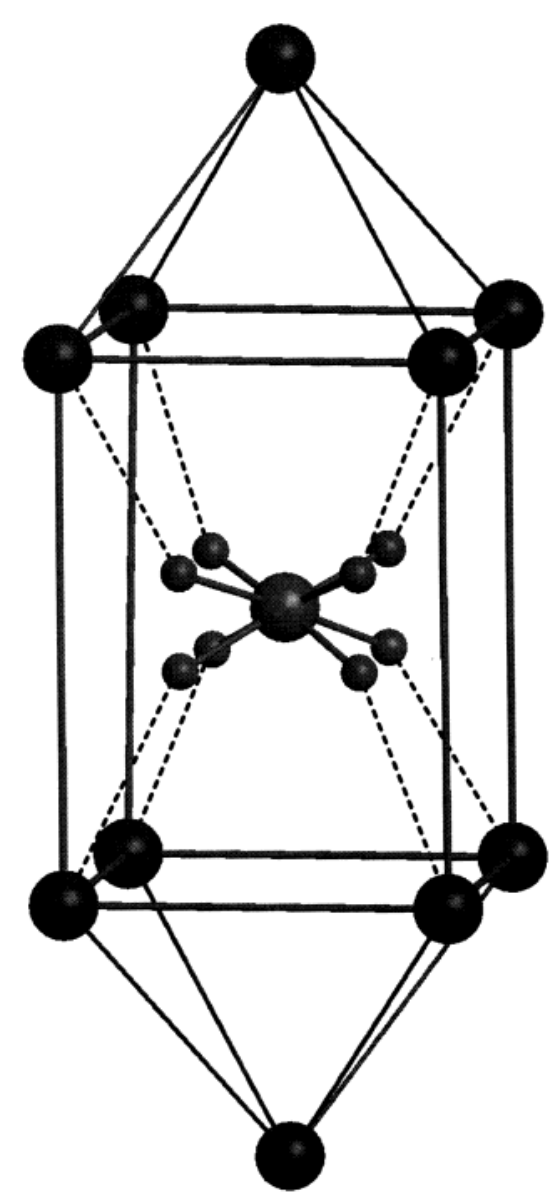

Figure 5. Time-averaged structure of a protonic defect in perovskite-type oxides (cubic case), showing the eight orientations of the central hydroxide ion stabilized by a hydrogen-bond interaction with the eight next-nearest oxygen neighbors. ${ }^{186,199}$

limiting step in the considered perovskites. On the other hand, the intense red-shifted $\mathrm{OH}$-stretching absorptions in the IR spectra (ref 197 and references therein) and the results of neutron diffraction experiments ${ }^{198}$ are indicative of strong hydrogen bond interactions, which favor fast proton-transfer reactions rather than fast reorientation processes, the latter requiring the breaking of such bonds.

Because the structural oxygen separation is larger than 290 pm in most perovskite-type oxides, and strong hydrogen bonds may only be formed at significantly shorter distances, the free energy that the system gains by hydrogen-bond formation is competing with the free energy required for the lattice distortion necessary for hydrogen bonding. A reanalysis of a quantum-MD simulation of a protonic defect in cubic $\mathrm{BaCeO}_{3}{ }^{131,188,199}$ demonstrated that these two free-energy contributions almost cancel each other for a wide range of oxygen separation distances ( 250-300 pm). Thus, short oxygen separations, which favor proton transfer, and large oxygen separations, which allow rapid bond breaking, result in similar free energies of the entire system; therefore, these separations have similar probabilities of occurring. Indeed, the simulation found the protonic defect to form short, but transient, hydrogen bonds with all eight nearest oxygen neighbors. In the time-averaged picture observed in the diffraction experiments (see
Figure 5), this leads only to a slight reduction in the structural $\mathrm{OH} / \mathrm{O}$ separations, in contrast to most instantaneous configurations where one of the eight $\mathrm{OH} / \mathrm{O}$ separations is reduced to $\sim 280 \mathrm{pm}$, because of hydrogen bonding. ${ }^{188,200}$ Although the hydrogen-bond interaction has a stabilizing effect of $\sim 0.5 \mathrm{eV}$ on this configuration, the bond is a "soft", high-energy hydrogen bond with extended bond-length fluctuations. This also leads to configurations, where the protonic defect acts almost like a free $\mathrm{OH}$ with small $\mathrm{OH}$ stretching amplitudes, compared to the extended stretching vibrations in the hydrogen bonded state. ${ }^{131}$

From the thermodynamics of such "dynamical hydrogen bonds", one may actually expect an activation enthalpy of long-range proton diffusion of not more than $0.15 \mathrm{eV}$, provided that the configuration $\mathrm{O}-\mathrm{H} \cdots \mathrm{O}$ is linear, for which the proton-transfer barrier vanishes at O/O distances of less than $\sim 250$ pm. However, the mobility of protonic defects in cubic perovskite-type oxides has activation enthalpies on the order of $0.4-0.6 \mathrm{eV} .{ }^{188}$ This raises the question as to which interactions control the activation enthalpy of proton transfer.

A more-detailed inspection of the MD data showed that, for most configurations with short $\mathrm{OH} / \mathrm{O}$ separation distances, the proton is not found between the two oxygens on the edge of the octahedron but outside the $\mathrm{BO}_{6}$ octahedron, as part of a strongly bent hydrogen bond ${ }^{200}$ that still possesses some barrier for proton transfer. The reason for this is probably the repulsive interaction between the proton and the highly charged B-site cation, which prevents a linear hydrogen bond from forming. The analysis of a few transition-state configurations showed that the $\mathrm{B}-\mathrm{O}$ bonds are elongated to some extent and that the displacement of the proton being transferred is on the edge of the distorted octahedron. ${ }^{131}$ In this way, an almost linear, short configuration of the type $\mathrm{O}-\mathrm{H} \cdots \mathrm{O}$ is formed. The proton transfer in this configuration probably occurs over a remaining barrier, as indicated by the experimentally observed H/D-isotope effects. ${ }^{201,202}$ Although the H/B repulsion is reduced in this configuration, major contributions to the activation enthalpy result from the $\mathrm{B}-\mathrm{O}$ bond el ongation and the proton-transfer barrier. Nevertheless, the $\mathrm{H} / \mathrm{B}$ repulsion may be used as an estimate of the upper limit for these contributions to the activation enthalpy.

The importance of the H/B repulsion is also witnessed by the observation that the activation enthalpies of proton mobility in cubic perovskites with pentavalent $\mathrm{B}$-site cations (I-V perovskites) are significantly higher than for perovskites with tetravalent B-site cations (II-IV perovskites). ${ }^{131,187}$

Similar to hydrogen-bonded networks, any reduction in symmetry may decrease the proton conductivity in oxides. This effect has been investigated in detail by comparing structural and dynamical features of protonic defects in yttrium-doped $\mathrm{BaCeO}_{3}$ and $\mathrm{SrCeO}_{3}{ }^{203}$ and $\mathrm{SrZrO}_{3}{ }^{204}$ The large orthorhombic distortion of $\mathrm{SrCeO}_{3}$ has tremendous effects on the arrangement of the lattice oxygen. The cubic oxygen site degenerates into two sites $(01,02)$ with different acid/base properties. Although, in $\mathrm{SrCeO}_{3}$, the most 
basic oxygen is $\mathrm{O} 1$, it is $\mathrm{O} 2$ in $\mathrm{BaCeO}_{3}$. Assuming that protons are associated with these sites for the majority of the time, they may show long-range proton transport via the most-frequent $\mathrm{O} 2$ sites in $\mathrm{BaCeO}_{3}$. This is in contrast to $\mathrm{SrCeO}_{3}$, where long-range proton transport must involve transfer between chemically different $\mathrm{O} 1$ and $\mathrm{O} 2$ sites. The latter, together with the observed bias in rotational diffusion (defect reorientation), was suggested as the reason behind the higher activation enthalpy and lower conductivity in $\mathrm{SrCeO}_{3}$, compared to $\mathrm{BaCeO}_{3}{ }^{203}$

The mobility of protonic defects was shown to be very sensitive not only to reduction in the crystallographic symmetry but also to local structural and chemical perturbations induced by the acceptor dopant or by mixed occupancy on the B-site. Traditionally, aliovalent dopants with matching ionic radii are chosen, and, indeed, this simple concept has proven to be successful; e.g., in the devel opment of oxide ion conductors. However, when it comes to proton conductivity in oxides, this approach clearly fails. Although $\mathrm{Sc}^{3+}$ and $\mathrm{In}^{3+}$ have similar ionic radii to $\mathrm{Zr}^{4+}$, $\mathrm{BaZrO}_{3}$ shows much lower proton mobility when doped with scandium or indium, compared to yttrium as an acceptor dopant with a significantly higher ionic radius. For the latter, the proton mobility and corresponding activation enthalpy are virtually independent of the dopant concentration. Electronic structure calculations show a significant effect of the acceptor dopant on the electron density of the neighboring oxygen, including its affinity for the proton (O2). Obviously, the chemical match of the dopant in yttrium-doped $\mathrm{BaZrO}_{3}$ makes it "invisible" to the diffusing proton. ${ }^{205}$ However, the most common observation is decreasing proton mobility and increasing activation enthalpy with increasing dopant concentration; e.g., as observed in yttrium-doped $\mathrm{BaCeO}_{3}{ }^{206}$ Thus, it is not surprising that mixed occupancy of the B-site in complex perovskites may become unfavorable for proton mobility especially when cation ordering occurs. ${ }^{207}$

The aforementioned considerations provide a qualitative explanation for the empirical finding that the highest proton conductivities are observed in oxides with a perovskite structure. The framework of cornersharing octahedral $\mathrm{BO}_{6}$ shows high coordination numbers for both cation sites (12 for the A-site and 6 for the B-site). There is only one oxygen site in the ideal perovskite structure that has each $\mathrm{O}$ atom surrounded by eight nearest and four next-nearest oxygens. Generally the high coordination numbers lead to low bond strengths and smaller angles between the bonds, which is in favor of the abovedescribed dynamics (e.g., the rotational diffusion of the protonic defect corresponds to "dynamical hydrogen bonding" of the $\mathrm{OH}$, with the eight $\mathrm{O}$ atoms forming the "reaction cage" (see Figure 5)). If the angles between the possible orientations are small enough, the effective barriers for bond breaking and forming processes are usually $<0.2 \mathrm{eV}$ for perovskites with lattice constants that are not too small. In these cases, hydrogen bonding even to the next-nearest oxygen between the vertices of the octahedral become possible, opening another proton-transfer path- way, as observed in MD simulations of protons in $\mathrm{CaTiO}_{3 .}{ }^{199}$

\subsubsection{Heterogeneous Systems (Confinement Effects)}

Homogeneous media have actually been used in commercial (e.g., phosphoric acid fuel cells (PAFCs), AFC) and laboratory fuel cells (e.g., sulfuric acidbased direct methanol fuel cells (DMFCs), hydrogen fuel cells with $\mathrm{CsHSO}_{4}$ as an electrolyte); however, most modern low-temperature fuel cells rely on the properties of heterogeneous separator materials such as hydrated sulfonic acid functionalized polymers (e.g., Nafion) and the adducts of basic polymers with oxo-acids (e.g., in the system of polybenzimidazole and phosphoric acid $\left.\left(\mathrm{PBI}-\mathrm{H}_{3} \mathrm{PO}_{4}\right)\right)$. In these materials, the homogeneous media (discussed previously) are confined within another phase. This geometric situation, which also comprises specific interactions at the usually very large internal interface, not only modifies the transport behavior within the protonconducting phase, but also leads to the appearance of new transport features, such as electro-osmotic drag (see Section 3.2.1.1). This section reviews the current understanding of proton conduction in these heterogeneous materials.

3.1.2.1. Hydrated Acidic Polymers. Hydrated acidic polymers are, by far, the most commonly used separator materials for low-temperature fuel cells. Their typical nanoseparation (also see Section 1) leads to the formation of interpenetrating hydrophobic and hydrophilic domains; the hydrophobic domain gives the membrane its morphological stability, whereas the hydrated hydrophilic domain facilitates the conduction of protons. Over the past few years, the understanding of the microstructure of these materials has been continuously growing, and this has been crucial for the improved understanding of the mechanism of proton conduction and the observed dependence of the conductivity on solvent (water and methanol) content and temperature.

The microstructure has chiefly been investigated through SAXS and small-angle neutron scattering (SANS) experiments. However, because such experiments on specific samples do not provide sufficient information, diffraction experiments on samples pre pared over a wide range of polymer/solvent ratios (even with different types of solvents) have been performed, especially by Gebel and co-workers. ${ }^{208-211}$ Additional information from water transport measurements has been included into the parametrization of the most simple microstructural model, assuming a hydrophobic matrix with a cubic system of cylindrical hydrophilic channels. ${ }^{212,213}$ This approach, although highly simplifying, has the advantage that it allows comparison of the mean numbers for the extensions of the hydrophilic channels and the hydrophobic matrix between for different types of ionomers (al so see Section 3.2.1.1). Other constraints such as "maximum entropy" (minimum structure) have also been used to obtain microstructural information from two-dimensional diffraction patterns by direct Fourier synthesis. ${ }^{214}$ In addition, a recent theoretical investigation by Khalatur et al. ${ }^{215}$ that implemented a hybrid Monte Carlo/reference interac- 


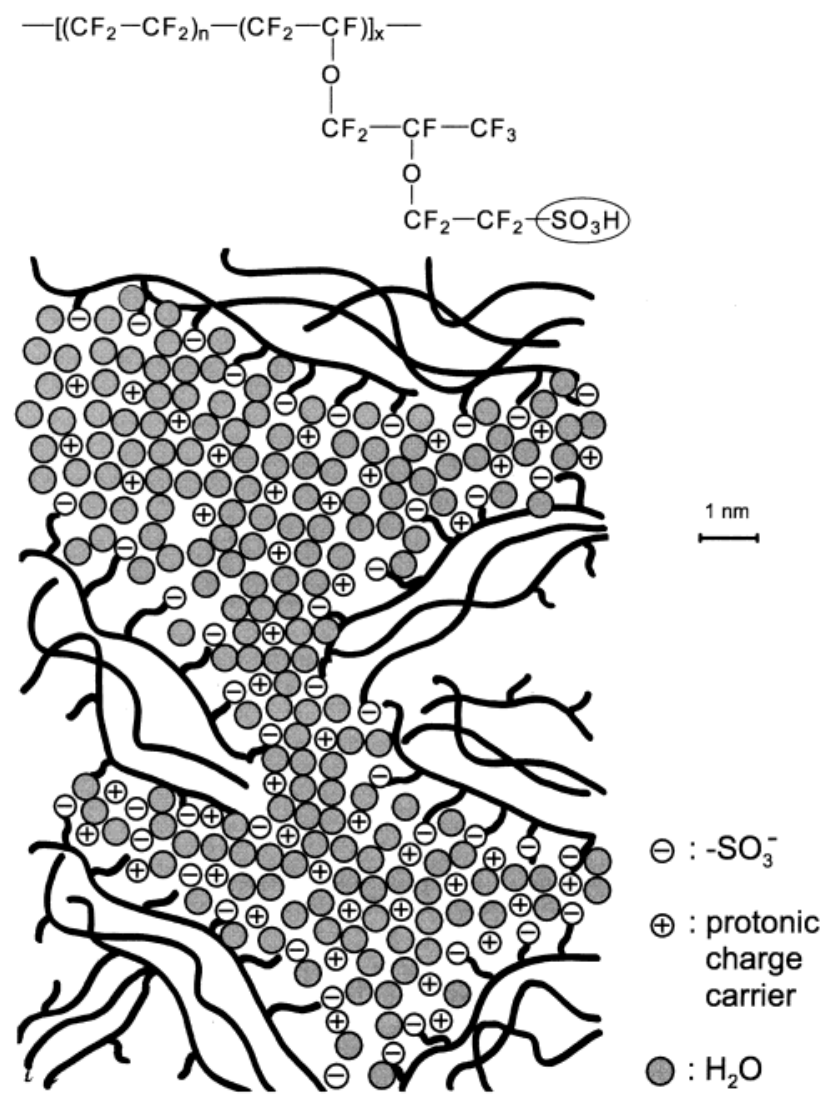

Figure 6. Two-dimensional illustration of some microstructural features of Nafion for an intermediate water content (see text).

tion site model (MC/RISM) technique to probe the morphology of Nafion over a range of hydration levels showed that a continuous network of channels might exist, even at very low water contents. Based on proton conductivity and water diffusion data obtained on diverse membrane materials, Edmondson and Fontanella claimed the existence of a universal percolation threshold at a water volume fraction of $\sim 5 \%$. ${ }^{216}$ This conclusion was based on the power-law behavior of the transport coefficients above this water concentration. The observations that the membrane materials continue to conduct bel ow the "threshold" and that the conduction mechanism changes with water content (see bel ow) leave serious doubts about the validity of this interpretation. However, the morphologies resulting from most approaches are in reasonable agreement for the most widely investigated Nafion (1100 g/equiv); the principal microstructural features of this morphology are illustrated in Figure 6 for an intermediate water content. The hydrophobic domain may be described by a framework of low-dimensional objects defining the boundary with the hydrophilic domain. In Nafion, the latter is well-connected, even at low degrees of hydration; i.e., there are almost no dead-end pockets and very good percolation. Because of the side-chain architecture of $\mathrm{N}$ afion, a third transition region between the aqueous domain and the hydrophobic polymer backbone has been introduced. This comprises the hydrated side chains, and a recent SAXS study seems to suggest that this region swells at the expense of the purely aqueous region with increasing degree of

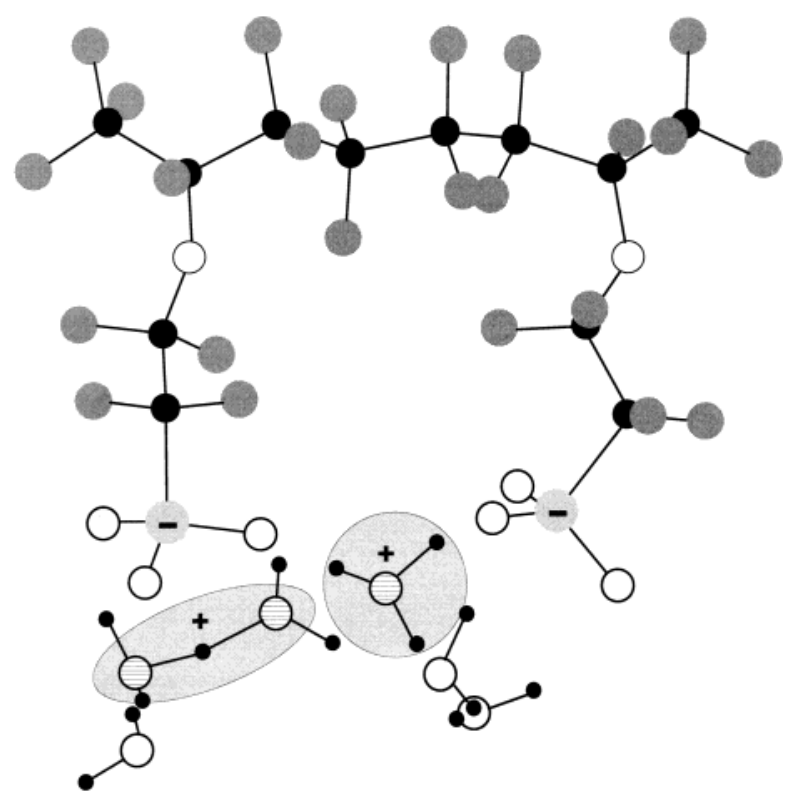

Figure 7. Minimum energy conformation of a two-sidechain fragment of a perfluoro sulfonic acid polymer (Dow) with six water molecules, showing the dissociation of both acidic protons. ${ }^{23}$

hydration. 217 In other words, there is indication for a progressive side-chain unfolding with increasing hydration. This idea is supported by electronic structure calculations (described in Section 2.1) on the entire Nafion side chain, ${ }^{18}$ which showed that the unfolding of the chain would require $\sim 18 \mathrm{~kJ} / \mathrm{mol}$.

Because the hydrophilic sulfonic acid groups are covalently bound to the hydrophobic polymer, they aggregate somewhere in the hydrophobic/hydrophilic transition region, with an average separation of $\sim 0.8$ $\mathrm{nm}$, compared to $\sim 1 \mathrm{~nm}$ expected for a totally uniform distribution within the material. ${ }^{212}$

Electronic structure calculations have shown that only 2-3 water molecules per sulfonic acid group are necessary for dissociation, in accordance with its superacidity when bound to a perfluorinated polymer (Figure 7), and when 6 water molecules are added, separation of the dissociated proton from the sulfonate anion is observed. ${ }^{16,20,23}$ This water (primary hydration of the sulfonic acid group) is actually absorbed at low water partial pressures, ${ }^{197}$ indicating the stabilizing effect of water in such systems, as expected from the high energetic stability (at $\mathrm{T}=0$ $\mathrm{K}$ ) of the $\mathrm{H}_{3} \mathrm{O}^{+}$, compared to $\mathrm{H}_{3} \mathrm{SO}_{4}{ }^{+}\left(-\mathrm{SO}_{3} \mathrm{H}_{2}{ }^{+}\right)^{218}$ Apart from water, the hydrophilic domain contains only excess protons as mobile species, while the anionic counter charge is immobilized. This is an inherent advantage of such materials over homogeneous electrolytes with mobile anions (or other conjugated bases), which may interfere with the reactions that occur at the el ectrocatalysts. Although this situation is reminiscent of the simplest case discussed in Section 3.1.1.1.1 (excess proton in water), it is actually more complex.

If only electrostatics are considered, significant attractive interaction between the excess protons in the aqueous phase and the immobile anionic mirror charge is expected. ${ }^{26,27}$ Recent MD simulations of proton transport in slab pores with sulfonate groups 
embedded on the pore walls give the anticipated, essentially constant separation distance of the protons from the fixed anions, despite changes in the amount of water between the slabs. The Debye length (typical electrostatic screening length) of pure water is $\sim 800 \mathrm{~nm}$ at room temperature and is thus much larger than the typical dimensions of the hydrophilic domain (only a few nanometers). Traditionally, the distribution of charge carriers within the corresponding space charge layer is described by the GouyChapman theory, which has been devel oped for semiinfinite geometries, or by numerically solving the Poisson-Boltzmann (PB) equation for specific geometries. ${ }^{99,100,212,219,220}$ In either case, one obtains a monotonically decreasing concentration of protonic charge carriers as one moves from the hydrophobic/ hydrophilic interface (i.e., where the anion charge resides) toward the center of the hydrated hydrophilic domain. However, this picture is not complete, because these continuum theories neglect any structural inhomogeneity in the vicinity of the electrified interface. In the Gouy-Chapman approach, even a homogeneous distribution of the mirror charge over the interface is assumed; however, the fact that the separation of neighboring sulfonic acid groups ( $\sim 0.8$ $\mathrm{nm}$ ) and the typical extension of the hydrophilic domain (a few nanometers) are of similar order does not justify this assumption. In addition, recent Brownian dynamics simulations of ions in cylindrical nanodimensioned pores have shown that both the PB and Poisson-Nernst-Planck continuum theories substantially overestimate the shiel ding effects when the radius is less than two Debye lengths. ${ }^{221}$

The same is true for the assumption of a homogeneous dielectric constant of the aqueous phase: a simplification that is not backed up by dielectric measurements as a function of the water content in the microwave (i.e., gigahertz) range. ${ }^{222,223}$ As known for the near surface region of bulk water or any interface with water on one side, the dielectric constant of the hydrated hydrophilic phase is significantly reduced near the hydrophobic/hydrophilic interface. In addition, the specific interaction of the sulfonic acid group with water (hydration) also decreases the dielectric constant. Therefore, the spatial distribution of the dielectric constant within hydrated domains is strongly dependent on the width of the channels (degree of hydration) and the separation of the dissociated sulfonic acid functional groups. This is an important result from the equilibrium statistical thermodynamic modeling of the dielectric saturation in different types of hydrated polymers described previously (see Section 2.5). ${ }^{47-51}$ As shown in Figure 8 (top), the dielectric constant reaches the bulk value (81) in the center of the channel (pore) for water contents higher than $\sim 10$ water molecules per sulfonic acid group, whereas, for lower degrees of hydration, even in the center of the channel, the dielectric constant is lower than the bulk value as a consequence of the stronger confinement (Figure 8, bottom). The calculations did not account for specific chemical water-polymer interactions (modeling only the interaction of the sulfonate groups with the water), which are expected to further reduce the dielectric constant within the hydrated channels. The distribution of the relative permittivity of the water across a hydrated channel has important implications on the distribution of excess protons within the channel. Because the solvation energy of protons becomes more negative with increasing dielectric constant, there is a stabilizing effect for protonic charge carriers in the center of the channels, which heavily modifies the Gouy-Chapman distribution. As illustrated in Figure 8 (top), the decreased dielectric constant in the interfacial region leads to a relative stabilization of the dissociated protons in the central region of the channels. Only at very high water contents (i.e., in the two-phase regime of $\mathrm{N}$ afion with more than 14 water molecules per sulfonic acid group) does one expect a slight relative depletion of charge carriers in the channel center, which is reminiscent of a Gouy-Chapman profile.

The general picture is such that the majority of excess protons are located in the central part of the hydrated hydrophilic nanochannels. In this region, the water is bulklike (for not too low degrees of hydration) with local proton transport properties similar to those described for water in Section 3.1.1.1.1. Therefore, the transport properties are indeed a function of the considered length and time scales, ${ }^{224,225}$ and the activation enthalpies of both proton mobility and water diffusion are similar to those of bulk water and only increase slightly with decreasing degree of hydration for intermediate water contents (Figure 9). 197,224,226-228

Apart from the slight retardation of proton mobility $\left(\mathrm{D}_{\sigma}\right)$ and water diffusion $\left(\mathrm{D}_{\mathrm{H}_{2} \mathrm{O}}\right)$ within the hydrophilic domain, the decrease in the transport coefficients with decreasing degree of hydration mainly reflects on the decreasing percolation within the waterlike domain. At the highest degrees of hydration, the major proton conduction mechanism is structure diffusion $\left(\mathrm{D}_{\sigma}>\mathrm{D}_{\mathrm{H}_{2} \mathrm{O}}\right.$, Figure 9). With decreasing water content, the concentration of excess protons in the aqueous phase is increasing, which, in turn, increasingly suppresses intermolecular proton transfer and, therefore, structural diffusion, as witnessed in aqueous solutions ${ }^{141}$ (see Figure 2 and Section 3.1.1.1.1). Consequently, proton mobility at intermediate and low degrees of hydration is essentially vehicular in nature. Nonequilibrium statistical mechanics-based calculations (see Section 2.3) of the proton self-diffusion coefficients in Nafion and PEEKK membranes over a range of hydration conditions have addressed this conductivity contribution, and they clearly show that the diffusion of water (vehicle) and hydrated protons $\left(\mathrm{H}_{3} \mathrm{O}^{+}\right)$are retarded, as a result of confinement in an environment where the water and protons are perturbed by the presence of a substantial density of sulfonate (i.e., negative charge) groups. 45,46 These calculations also showed that structure diffusion contributes to the diffusion of protons at the higher water contents (i.e., > 13 water molecules per sulfonic acid group; see Section 2.4). ${ }^{20,22,23}$ The very high conductivity activation volumes (conductivity decreases with applied pressure $(-k T$ a In $\sigma / \partial$ In p)), which are particularly evident in well-separated perfluorosulfonic acid polymers 


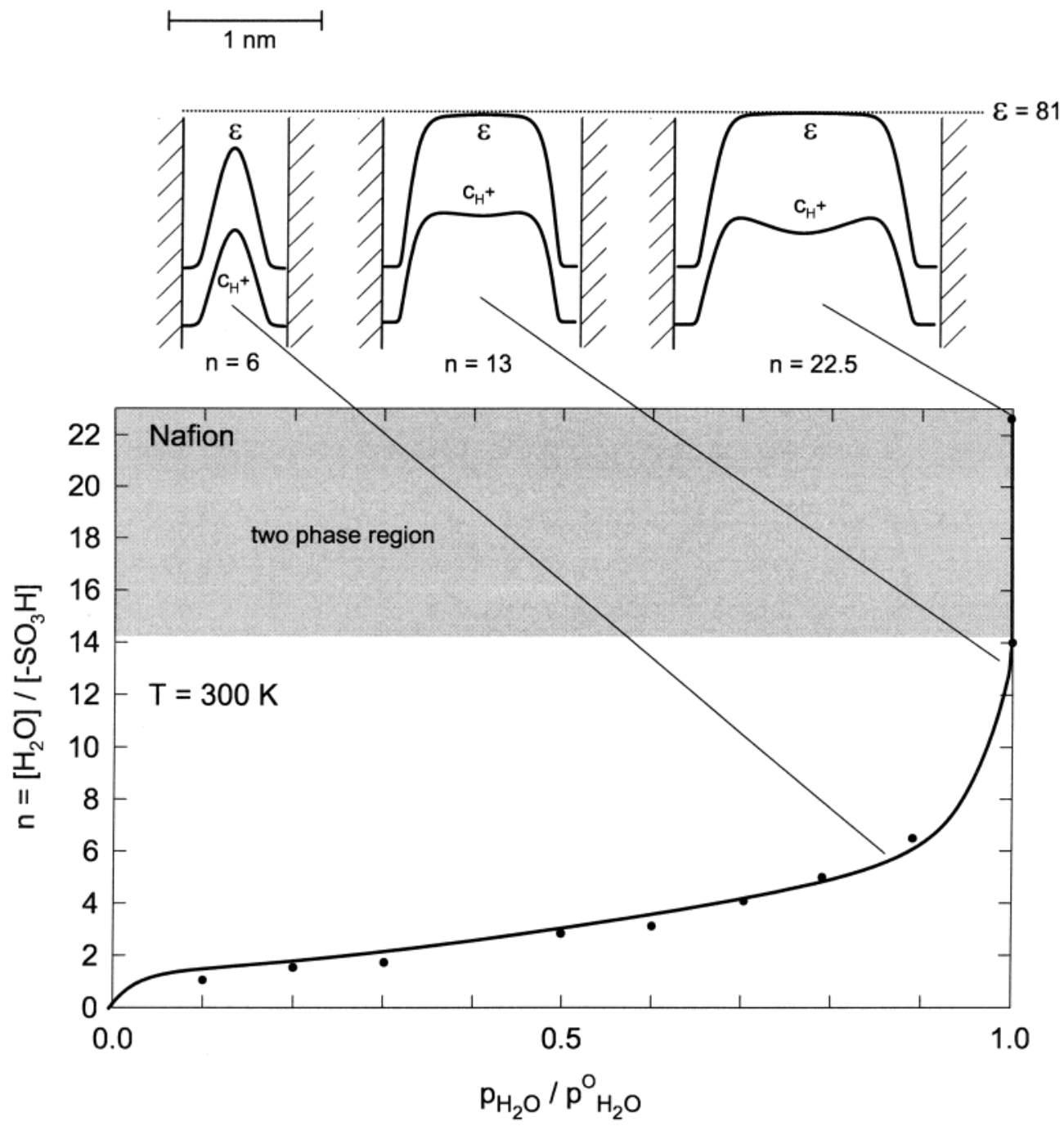

Figure 8. Hydration isotherm for Nafion 117 (equivalent weight (EW) of 1100 g/equiv) 197 and the distribution of the dielectric constant 23 and protonic charge carrier concentration across the hydrated hydrophilic channels (pores) for three different water contents (top).

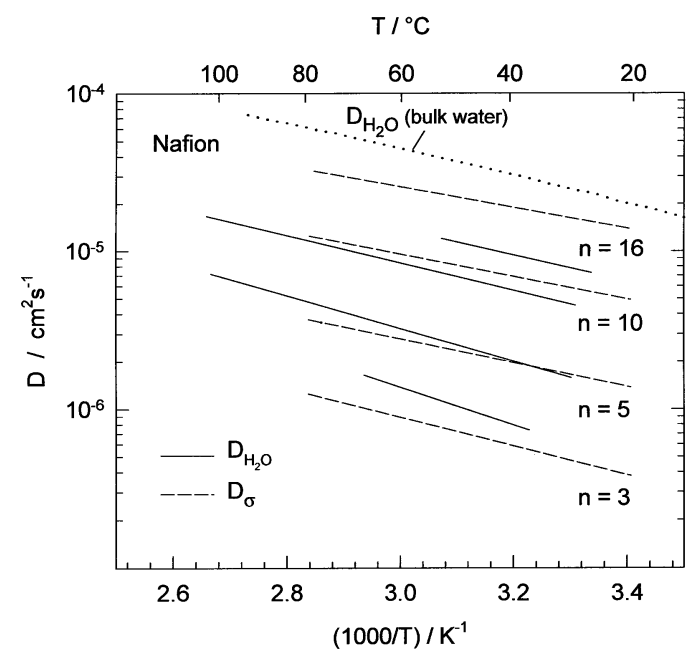

Figure 9. Proton conductivity diffusion coefficient (mobility) and water self-diffusion coefficient of Nafion 117 (EW $=1100 \mathrm{~g}$ (equiv), as a function of temperature and the degree of hydration $\left(n=\left[\mathrm{H}_{2} \mathrm{O}\right] /\left[-\mathrm{SO}_{3} \mathrm{H}\right]\right) .{ }^{197}$

(Nafion, Dow), ${ }^{216}$ may then also be understood as a confinement effect. Pressure probably leads to an increasing dispersion of the water, i.e., the hydro- phobicand hydrated hydrophobicdomainsare "squeezed" into each other, forming a more dendritic microstructure with narrower hydrophilic channels. This view explains straightforwardly why conductivity activation volumes in less-separated (better-dispersed) hydrocarbon-based membranes ${ }^{213}$ are significantly smaller. As long as the confinement does not fall bel ow $\sim 1 \mathrm{~nm}$, the water at the center of the channels may still be bulklike (Figure 8), and the diffusion of hydrated protons has a large hydrodynamic component with significant long-range velocity correlations, typical for viscous media. This feature will be addressed later in this article to explain the unusually high electro-osmotic drag coefficients (Section 3.2.1.1).

For very low degrees of hydration (i.e., for Nafion membranes with $<6$ water molecules per sulfonic acid group), the decreasing solvent (water) activity leads to a decreasing dissociation of the sulfonic acid group, i.e., an increasing exclusion of protons from the transport in the aqueous phase. Changes in the neighboring chemical group to the sulfonic acid (i.e., changing from a perfluoro methylene to an aromatic carbon: Nafion to S-PEK) will also affect the dissociation and separation of the proton from the acidic group. ${ }^{20}$ This effect is dependent not only on the 
acidity of the sulfonic acid group, but also on the dielectric constant of the water of hydration. With this understanding, the significant decrease of conductivity in the presence of methanol, ${ }^{219}$ which actually exhibits similar self-diffusion coefficients as water (see Section 3.2.1.1) but with a lower dielectric constant, may then be explained by increased ion pairing (decreasing dissociation).

3.1.2.2. Adducts of Basic Polymers with Oxoacids. To date, the most relevant materials of this type are adducts (complexes) of polybenzimidazole (PBI) and phosphoric acid, as introduced by Wainright et al. ${ }^{229}$ In contrast to water, which exhibits a high mobility for protonic defects but a very low intrinsic concentration of protonic charge carriers, phosphoric acid shows both high mobility and a high concentration of intrinsic protonic defects (see Section 3.1.1.2). In other words, phosphoric acid is intrinsically a very good proton conductor with a very small Debye length, and its charge carrier density is hardly affected by the interaction with PBI. I ndeed, a strong acid/base reaction occurs between the nonprotonated, basic nitrogen of the PBI repeat unit and the first phosphoric acid absorbed. The transfer of one proton leads to the formation of a benzimidazolium cation and a dihydrogenphosphate anion, forming a stable hydrogen-bonded complex, as shown by infrared spectroscopy. 230,231 It is a common observation for all systems of this type that their conductivity strongly increases upon further addition of an oxo-acid approaching the conductivity of the pure acid for high acid concentrations (recently free-standing films of $\mathrm{PBI} \cdot \mathrm{nH}_{3} \mathrm{PO}_{4}$ with extremely high acid-to-polymer ratios of $>10$ have been reported). ${ }^{232}$ In particular, there is no indication of participation of the polymer in the conduction process (also see discussion below).

Although no microstructural information is available to date, the macroscopic transport has been investigated in the related system of poly(diallyldimethylammonium-dihydrogenphosphate) and phosphoric acid, (PAMA $\left.{ }^{+} \mathrm{H}_{2} \mathrm{PO}_{4}^{-}\right) \cdot \mathrm{nH}_{3} \mathrm{PO}_{4}{ }^{233}$ The proton mobility $\left(\mathrm{D}_{\sigma}\right)$ and the self-diffusion coefficient of phosphorus $\left(D_{P}\right)$, as a measure of the hydrodynamic diffusion of the system, is shown in Figure 10 for a given temperature, as a function of the polymer/acid ratio. Similar to pure phosphoric acid, the mobility of protonic charge carriers is significantly higher than the self-diffusion coefficient of the phosphate species and both transport coefficients decrease with increasing polymer content virtually in the same manner. The main effect, obviously, is just the decreasing percolation within the liquidlike portion of the phosphoric acid domain, which is reminiscent of the situation in hydrated acidic polymers (see Figure 6). At very small acid contents, when all the phosphoric acid is immobilized in the 1:1 complex, only very little conductivity is left.

Similar to that observed for pure phosphoric acid, the transport properties of PBI and phosphoric acid are also dependent on the water activity, i.e., on the degree of condensation (polyphosphate formation) and hydrolysis. There is even indication that these reactions do not necessarily lead to thermodynamic equilibrium, and hydrated orthophosphoric acid may

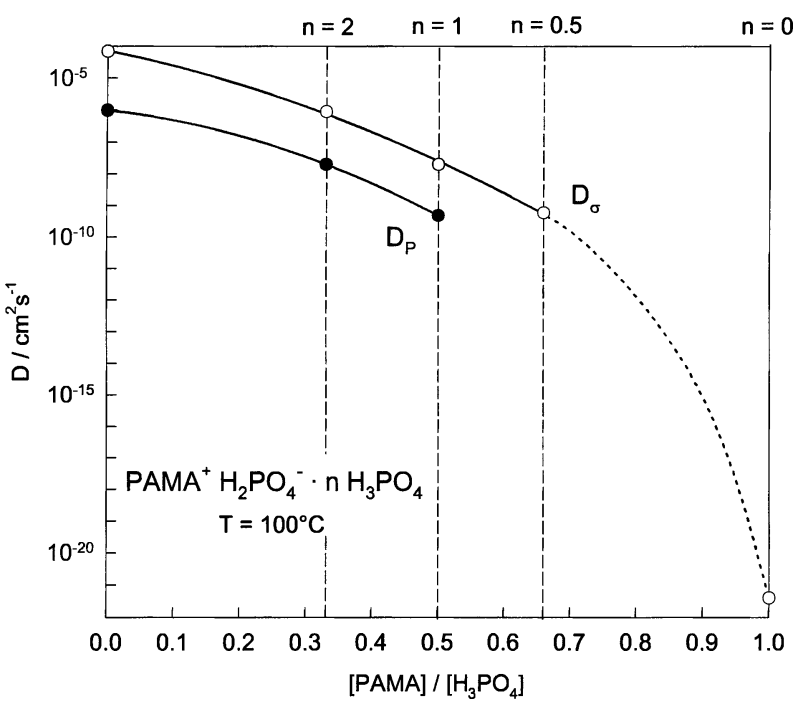

Figure 10. Proton conductivity diffusion coefficient (mobility) and self-diffusion coefficient of phosphorus for poly(diallyldimethylammonium-dihydrogenphosphate)-phosphoric acid ((PAMA $\left.\left.{ }^{+} \mathrm{H}_{2} \mathrm{PO}_{4}^{-}\right) \cdot \mathrm{nH}_{3} \mathrm{PO}_{4}\right)$, as a function of the phosphoric acid content. 233 Note that the ratio $\mathrm{D}_{\sigma} / \mathrm{D}_{\mathrm{P}}$ remains almost constant (see text).

coexist with polyphosphates in heterogeneous gel-like microstructures. ${ }^{232}$ Not much is known on the mechanism of proton transport in polymer adducts with polyphosphates and/or low hydrates of orthophosphoric acid. The determination of whether the increased conductivity at high water activities is the result of the "plasticizing effect" of the water on the phosphate dynamics and thereby assisting proton transfer from one phosphate to the other, or whether the water is directly involved in the conduction mechanism, has not been elucidated.

3.1.2.3. Separated Systems with I mmobilized Proton Solvents. Both types of heterogeneous systems discussed previously are comprised of a polymeric domain and a low-molecular-weight liquidlike domain (e.g., $\mathrm{H}_{2} \mathrm{O}, \mathrm{H}_{3} \mathrm{PO}_{4}$ ) with weak ionic or hydrogen-bond interaction between the two domains. Apart from other polar solvents, heterocycles such as imidazole, pyrazole, and benzimidazole have been intercalated into sulfonated polymers, ${ }^{170,171,234,235}$ re sulting in similar transport properties, as discussed for the hydrated systems in Section 3.1.1.1, although at somewhat higher temperatures. Apart from the proton donor and acceptor sites $(\mathrm{N})$, such solvents contain additional sites (C), which may be used for covalent "grafting" to oligomeric or polymeric structures. If these are hydrophobic (nonpolar), a similar separation as that described in Section 3.1.1.1 may occur, however with covalent bonding bridging the nonpolar/polar "interface". This approach has been implemented to obtain systems with completely immobilized proton solvents that still exhibit high proton conductivity with structure diffusion as the sole proton conduction mechanism (see Figure 3). Of course, the covalent bonding across the nonpolar/ polar interface mediates a significant influence of the nonpolar portion of the structure on the structure and dynamics of the polar proton-conducting domain. This cannot be approximated by simple percolation effects, as discussed for adducts of basic polymers 
with oxo-acids (see also Figure 10), but rather in a more complex fashion, involving (i) the position and character of the covalent bonding between the polar solvent and the nonpolar portion of the structure, (ii) the softness of this structure, and (iii) the volume fraction of the polar solvents.

As described in Section 3.1.1.3, the two nitrogens of the heterocycles act equally as proton donors or acceptors. Any covalent immobilization must avoid reduction of this symmetry, which is best achieved using the carbon between the two nitrogens (i.e., C2 in imidazole or C4 in pyrazole) for covalent bonding. Interestingly, this is not the case in histidine, which is an imidazole containing amino acid, which is frequently involved in proton translocation processes. However, the energetic asymmetry is very small ( 20 $\mathrm{meV}$ ) in this particular case. ${ }^{236}$ The type of bonding seems to be much more important, i.e., only single bonds allow reorientation of the bonded heterocycle, which is a persistent element in the proton conduction mechanism (see Section 3.1.1.3). To minimize the constraints in the dynamical aggregation of the heterocycles, immobilization via flexible spacers, such as alkanes or ethylene oxide (EO) segments, seems to be favored.213,237 The optimum spacer length is then given by the optimum balance between heterocycle aggregation and heterocycle density, on one hand, and the dynamics of the hydrogen bond network formed by the heterocycles on the other hand. The compound 2,2'-bis(imidazole), which is a brittle solid with a high melting point, is perfectly aggregated by strong static hydrogen bonding with negligible proton conductivity (according to recent results from the laboratories of one of the authors). Separating the two imidazoles by a soft EO spacer leads to the appearance of significant proton conductivity and a decrease in the melting point and glasstransition temperature $\left(T_{g}\right)$ with increasing spacer length. ${ }^{238,239}$ The conductivity then displays typical VTF behavior and, for a given concentration of excess protons (dopant), it is very similar for all spacer lengths when plotted versus $1 /\left(T-T_{0}\right)$, where $T_{0}$ is closely related to $\mathrm{T}_{\mathrm{g}}$. For very high spacer lengths, the dilution of the heterocycles by the spacer segments has a tendency to reduce aggregation of the heterocycles and, therefore, once again, reduce proton mobility.

The aggregation of imidazole leading to a continuous hydrogen-bonded structure in crystalline I mi-2 (two imidazole units spaced by two EO repeat units) is shown in Figure 11a. ${ }^{238} \mathrm{U}$ pon melting, the situation in most parts of the material is more similar to that shown in Figure 11b. This is one result coming from an NMR study ${ }^{240}$ demonstrating that liquid Imi-2 exhibits ordered domains (similar to the crystalline form), a dynamically disordered but still aggregated domain, and a certain fraction of nonbonded molecules. It is only within the disordered domain (Figure 11b) that fast proton mobility is observed, again demonstrating the delicate balance of aggregation and dynamics in hydrogen-bonded structures with high proton mobility.

Another interesting observation is that heterocycles immobilized in this way may still form dy- (a)

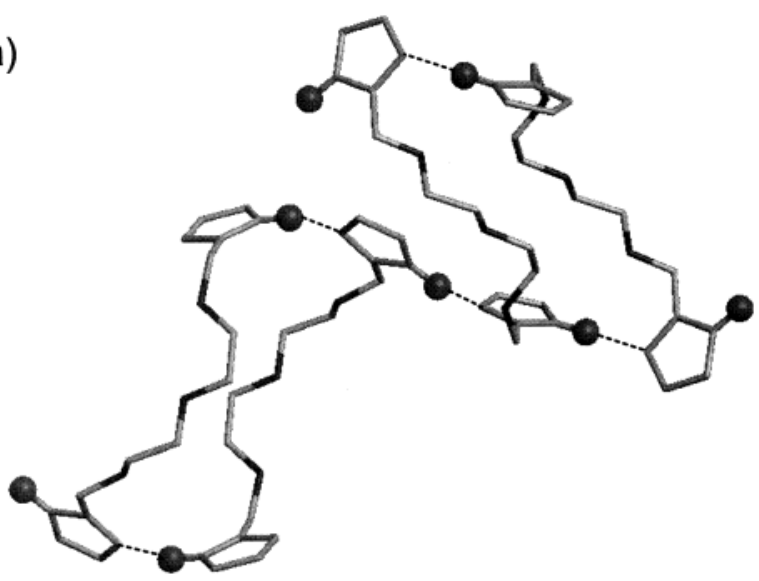

(b)

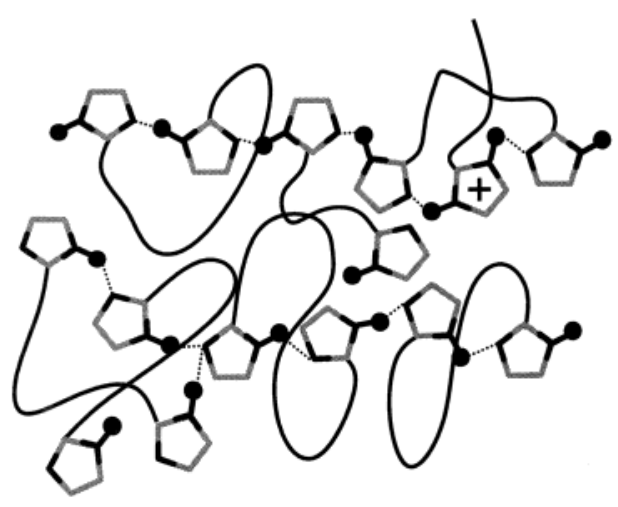

Figure 11. Hydrogen-bonded structure of Imi-2 (two imidazoles spaced by two ethylene oxide (EO) repeat units): (a) in the crystalline state, as revealed by an X-ray structure analysis, 239 and (b) in the liquid state (schematical), as suggested by an NMR study. 240 Note that the hydrogen bonds in the solid state are long-lived, whereas the hydrogen bonding in the molten state is highly dynamic (see text).

namical hydrogen-bond networks with very high proton mobility, although the self-diffusion of the heterocycle is significantly retarded. In the systems described previously, $\mathrm{D}_{\sigma}$ is typically 1 order of magnitude greater than $D_{\text {Imi }}$. Recently, fully polymeric systems with side-chain architectures have been developed that still exhibit high proton mobility, despite complete long-range immobilization of imidazole, i.e., $D_{\sigma} / D_{\text {Imi }}=\infty .{ }^{241}$ This finding is of paramount importance, because it demonstrates that complete decoupling of the long-range transport of protons and heterocycles (the proton solvent) is possible. The reader may recall that complexation of phosphoric acid and a basic polymer does not show any signature of this effect (see Figure 10), which may open the way to the development of true single ion conductors.

One of the problems associated with the use of heterocycles is that the intrinsic concentration of protonic charge carriers can only be moderately increased through acid doping (also see Section 4.3). This is particularly the case when the dynamics within the hydrogen-bonded domain is highly constrained through immobilization (especially in fully polymeric systems), which is probably the direct consequence of a reduced dielectric constant. This also leads to a further increase of the Haven ratio 
$\mathrm{D}_{\mathrm{H}} / \mathrm{D}_{\sigma}$, as discussed in Section 3.1.1.3 (also see Figure 4).

Also note that the spacer concept, as verified by several laboratories (e.g., see Perrson and J annasch ${ }^{242}$ ), has been moved forward to the immobilization of other proton solvents such as phosphonic acid, with very promising results (see Section 5).

3.1.2.4. Composites. Because of the drawbacks in hydrated acidic polymers for application in fuel cells, composites with highly dispersed inorganic phases have also been investigated, and, indeed, certain systems show improved performance in operating fuel cells.243,244 The extent that this is due to a modification of the bulk transport properties (in particular, the proton conduction mechanism) or of the membrane/electrode interface has not been conclusively determined. However, the similarities of the bulk transport properties of some modified and unmodified ionomers (see Section 4.1) are indicative of the possible relevance of interfacial effects. In view of the attention such systems are attracting at the moment, some general aspects are summarized here (for a review on the different type of systems, see, e.g., refs 245 and 246). Such composites are usually formed by either dispersing inorganic particles in a solution of the ionomer, followed by film casting and solvent elimination, or by precipitation of inorganic particles within a pre-existing membrane. The latter procedure was first used by Mauritz ${ }^{247}$ to precipitate $\mathrm{SiO}_{2}$ particles in Nafion using in situ hydrolysis of tetraethoxysilane (TEOS). Although $\mathrm{SiO}_{2}$ does not show measurable proton conductivity itself, other inorganic compounds, such as diverse zirconium phosphates and phosphonates or heteropolyacids, ${ }^{245,248}$ have recently been used as fillers. Although these materials possess some intrinsic proton conductivity, it is much lower than that of theionomeric host (e.g., Nafion) at low temperatures and high humidities. This is also true for the hydrophilicity ("waterretaining properties") of such inorganic particles, which is frequently thought to be responsible for the favorable performance of the corresponding composites in fuel cells. 234,249 There is actually no experimental evidence for this, and the strong acidity of perfluorosulfonic acid polymers is not expected to be exceeded by any of the aforementioned inorganics. Of course, this does not hold for the water uptake in contact with liquid water, which is sometimes taken as a measure of the water-retaining properties. ${ }^{250-252}$ The higher water uptake under these conditions may be the result of the reduction of the tensile strength of the polymer in the composite, allowing more water to enter as a second phase (which is actually not retained by any specific interaction). The most important effect of filling an ionomer with inorganic partides is probably the modification of the membrane/ electrode interface (see above), the microstructure and the elastic properties of the ionomeric component (with a glass transition temperature of $\mathrm{T}_{\mathrm{g}} \approx 120^{\circ} \mathrm{C}$ for plain Nafion, the latter may be a specific advantage of the use of composite membranes in this temperature range). These changes may also alter the transport properties in a similar way as discussed for the relationships between microstructure and transport in plain ionomers (see Sections 3.1.2.1 and 3.2.1). Particularly, in the case of proton-conducting zirconium phosphate prepared via in situ growth within the preformed membrane, 253 the proton conductivity of the highly dispersed filler may have some significance at high temperature and low humidity, where the conductivity of pure Nafion strongly decreases. ${ }^{245}$

\subsection{Mechanisms of Parasitic Transport}

The transport of protonic charge carriers is sometimes inherently connected to the transport of other species (e.g., in hydrated acidic polymers, Section 3.1.2.1). Sometimes, there are just indirect mechanistic relationships, or the existence of completely independent transport paths (e.g., protonic charge carriers and electronic holes in oxides). Parasitic transport frequently limits the fuel-cell performance, and a mechanistic understanding is definitely useful in the development of separator materials.

The advantageously low methanol "crossover" of $\mathrm{PBI}-\mathrm{H}_{3} \mathrm{PO}_{4}$ adducts is well-established and the problems related to its low oxygen transport are also well-described. ${ }^{254} \mathrm{H}$ owever, the fundamentals of these transport phenomena have not been subjected to any systematic investigation. For the recently emerging systems based on heterocycles and other proton solvents (also see Section 5), the investigation of parasitic transport is only just beginning. On the other hand, there is extensive literature on parasitic transport in the widely used hydrated acidic ionomers (especially Nafion). In addition, the transport properties of oxides have also been intensely investigated by groups mainly outside the fuel-cell community.

Therefore, the following discussion of the mechanisms of parasitic transport is restricted to only these two classes of materials.

\subsubsection{Solvated Acidic Polymers}

As discussed in Section 3.1.2.1, proton transport in acidic polymers occurs within a system of hydrated and connected hydrophilic pores (channels) within the nanoseparated materials. The extension of the channels, which, of course, shows some distribution within a given sample, may range from $<1 \mathrm{~nm}$ to several nanometers, depending on the type of polymer and the degree of solvation (swelling). This range covers the transition from more solidlike behavior with diffusive transport to a liquidlike regime with additional hydrodynamic transport (viscous flow). Apart from protonic defects, the main species that are confined to the hydrophilic domains are water and methanol (used as the fuel in DMFCs). The transport of these species is highly correlated and ther efore discussed together in the following section. The transport mechanisms of fuel-cell rel evant gases $\left(\mathrm{O}_{2}, \mathrm{H}_{2}\right)$, which may dissolve in the water domains and also in other parts of the nanostructures, are also reviewed in this section.

3.2.1.1. Mechanisms of Solvent (Water, Methanol) Transport. The following types of transport are considered in this section: (i) self-diffusion or tracer diffusion of solvent molecules, which is the unidirec- 


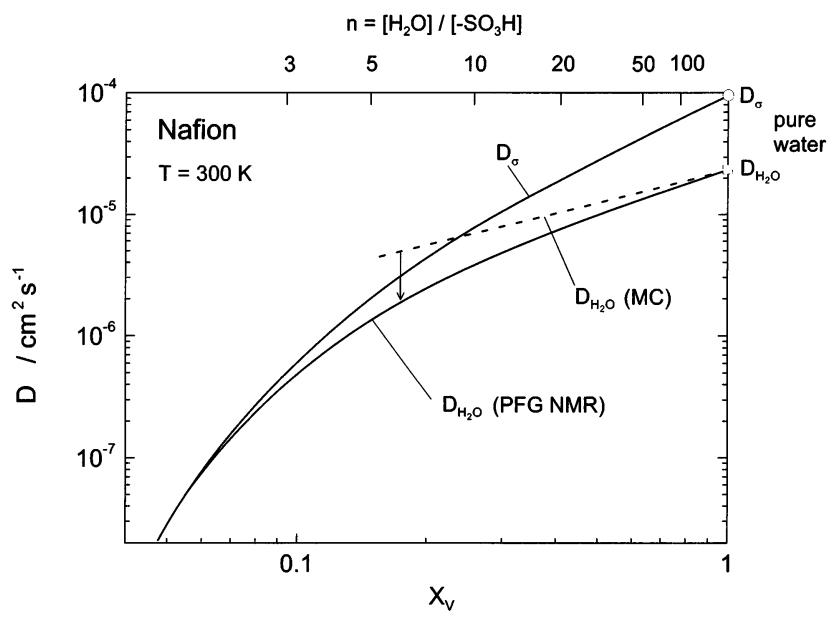

Figure 12. Water self-diffusion coefficient of Nafion 117 ( $E W=1100 \mathrm{~g} /$ equiv), as a function of the water volume fraction $X_{V}$ and the water diffusion coefficient obtained from a Monte Carlo (MC) simulation (see text). The proton conductivity diffusion coefficient (mobility) is given for comparison. The corresponding data points are displayed in Figure 14.

tional transport of "marked species" (e.g., this may be an isotopic tracer or the spin-labeled nuclei in PFG NMR experiments) with the thermal energy as the only driving force; (ii) chemical or Fickian diffusion, which is the average drift of a component $i$ in a chemical potential (approximately proportional to In $a_{i}$ or In $p_{i}$ ) gradient of the particular component related to the concentration gradient via the thermodynamical factor $\left(d\left(\ln a_{i}\right) / d\left(\ln c_{i}\right)\right)$; and (iii) permeation, which is the flow of a component (frequently solvent) in a total pressure gradient. Following this, the mechanisms of el ectro-osmotic drag are reviewed. This is the coupled transport of a protonic species and solvent molecules, i.e., the transport of neutral solvent in an el ectrical field with a vanishing gradient in the chemical potential. This phenomenon is symmetrical to the transport of protons in a chemical potential gradient of solvent in the absence of any electrical field. The coupling of water and methanol transport has not been studied explicitly; however, there is indirect indication of the nature of this coupling, which will also be discussed.

The self-diffusion coefficient of water in Nafion, as measured by PFG NMR, 197,224,226,255-261 is shown in Figure 12, as a function of the water volume fraction. At high degrees of solvation, this is only slightly lower than the diffusion coefficient obtained from MC simulations of random walk transport within nanostructures parametrized by results of SAXS experiments ${ }^{174,212,213}$ (see Figure 13) assuming the absence of any dead-end channels and a local diffusion coefficient that is identical to the bulk diffusion coefficient of water $\left(2.25 \times 10^{-5} \mathrm{~cm}^{2} / \mathrm{s}\right)$ at any position within the well-connected hydrophilic domain. This finding suggests that the major reason for a decrease in the water diffusion coefficient with decreasing water content is the decreasing percolation within the hydrophilic domain, especially at high water contents (also see Section 3.1.2.1). This is also supported by the observation that methanol diffusion follows the same trend (Figure 14a), 174,262,263 but with somewhat

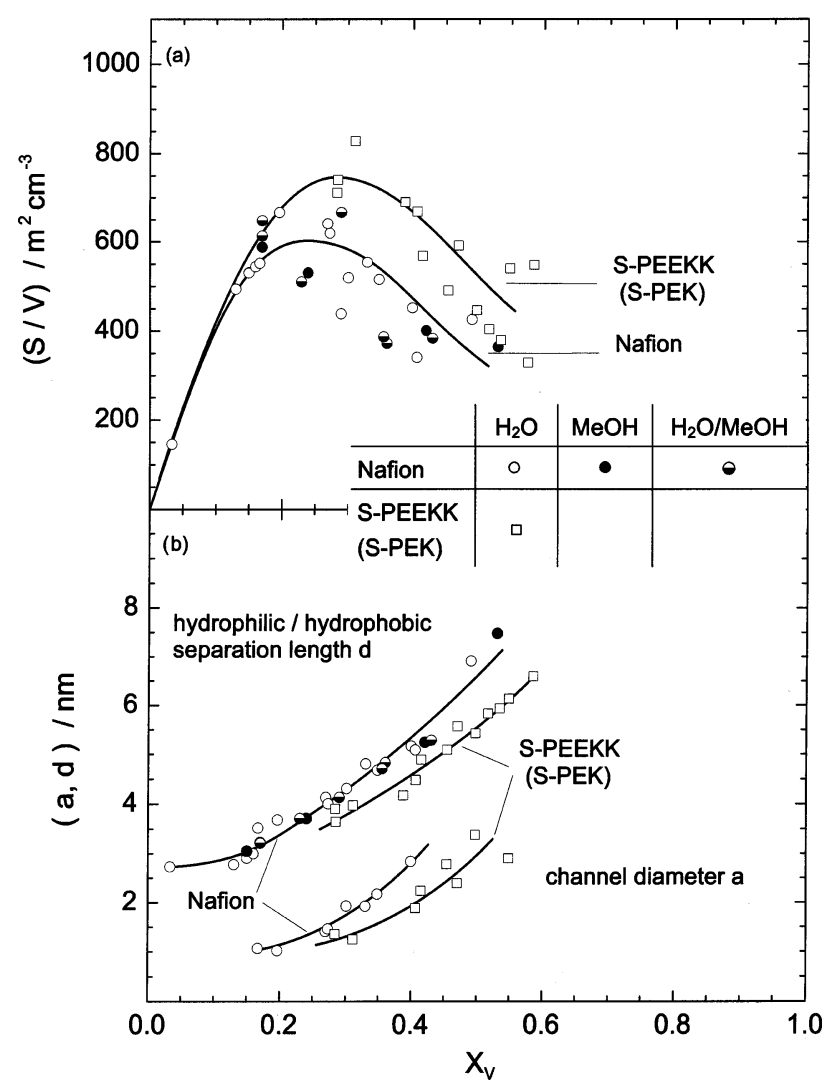

Figure 13. A few microstructural parameters for Nafion and sulfonated poly(arylene ether ketone)s, ${ }^{174,208-213}$ as a function of the solvent (water and/or methanol) volume fraction $X_{v}$ : (a) the internal hydrophobic/hydrophilic interface, and (b) the average hydrophobic/hydrophilic separation and the diameter of the solvated hydrophilic channels (pores).

lower diffusion coefficients that correspond to the lower self-diffusion coefficient in bulk methanol compared to that in bulk water. At low water contents, however, the experimental diffusion coefficient falls off more rapidly than the simulated one (see Figure 12). One reason is simply the fact that the connectivity within the solvated hydrophilic domains decreases (appearance of dead-end channels, pockets). ${ }^{212,213}$ Another reason is the retardation of water diffusion as a result of increasing confinement, which increases the internal molecular friction within the solvated domain as discussed above (see Sections 3.4 and 3.1.2.1). ${ }^{41-46} \mathrm{~A}$ comparison of Figures 12 and 13 suggests that this effect becomes particularly evident when the diameter of the channel decreases to $<1 \mathrm{~nm}$.

For comparison purposes, the proton mobility, $\mathrm{D}_{\sigma}$ (for Nafion solvated with water), which is closely related to the self-diffusion coefficient of water, is also plotted. At low degrees of hydration, where only hydrated protons (e.g., $\mathrm{H}_{3} \mathrm{O}^{+}$) are mobile, it has a tendency to fall below the water diffusion coefficient (this effect is even more pronounced in other polymers), which may be due to the stiffening of the water structure within the regions that contain excess protons, as discussed in Section 3.1.1.1.148 Interestingly, the proton mobility in Nafion solvated with methanol $\left(\mathrm{D}_{\sigma}(\mathrm{MeOH})\right.$ in Figure $\left.14 \mathrm{a}\right)$ is even lower than the methanol self-diffusion $\left(\mathrm{D}_{\mathrm{MeOH}}\right)$. This may 

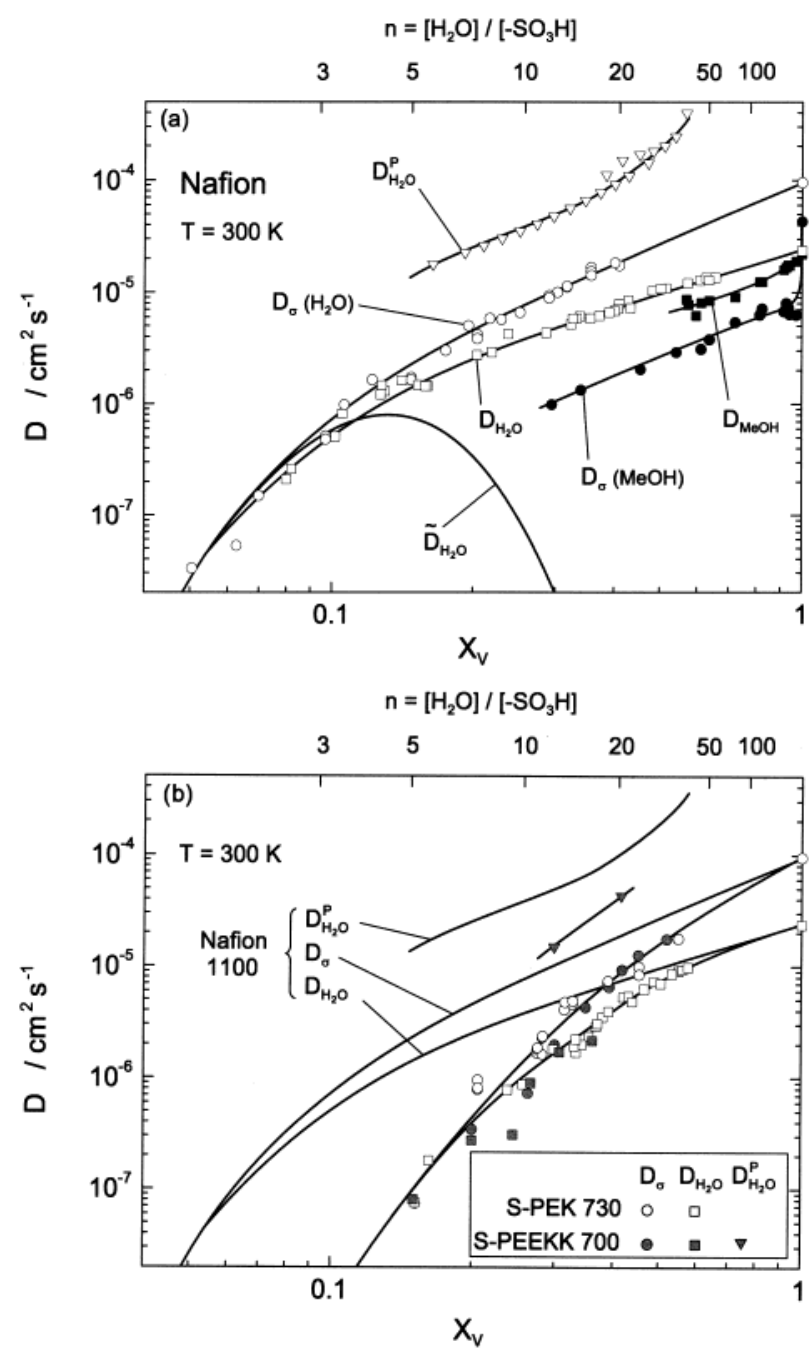

Figure 14. Solvent (water, methanol) diffusion coefficients of (a) Nafion 117 (EW = 1100 g/equiv) and (b) sulfonated poly(arylene ether ketone)s, as a function of the solvent volume fraction. Self-diffusion data $\left(\mathrm{D}_{\mathrm{H}_{2} \mathrm{O}}, \mathrm{D}_{\mathrm{MeOH}}\right)$ are taken from refs 197, 224, 226, 255-263 and unpublished data from the laboratory of one of the authors); chemical diffusion coefficients $\left(\tilde{\mathrm{D}}_{\mathrm{H}_{2} \mathrm{O}}\right)$ are calculated from self-diffusion coefficients (see text), and permeation diffusion coefficients are determined from permeation coefficients. 212,267,268 Proton conductivity diffusion coefficients for hydrated samples $\left(\mathrm{D}_{\sigma}\left(\mathrm{H}_{2} \mathrm{O}\right)\right)^{197,224,226,255-261}$ and samples solvated with methanol $\left(\mathrm{D}_{\sigma}(\mathrm{MeOH})^{256}\right)$ are given for comparison.

be the consequence of the lower dielectric constant of methanol, compared to water, especially under the condition of confinement in a perfluorinated ionomer (see discussion in Section 3.1.2.1 and Figure 8).

When it comes to the equilibration of water concentration gradients, the relevant transport coefficient is the chemical diffusi on coefficient, $\tilde{\mathrm{D}}_{\mathrm{H}_{2} \mathrm{O}}$. This parameter is related to the self-diffusion coefficient by the thermodynamic factor (see above) if the elementary transport mechanism is assumed to be the same. The hydration isotherm (see Figure 8) directly provides the driving force for chemical water diffusion. Under fuel-cell conditions, i.e., high degrees of hydration, the concentration of water in the membrane may change with only a small variation of the chemical potential of water. In the two-phase region (i.e., water contents of $>14$ water molecules per sulfonic acid), any driving force for Fickian water diffusion vanishes, i.e., there is no chemical diffusion. A fitting expression of $\tilde{\mathrm{D}}_{\mathrm{H}_{2} \mathrm{O}}$ is given in ref $264 ; \tilde{\mathrm{D}}_{\mathrm{H}_{2} \mathrm{O}}$ data, calculated from $\mathrm{D}_{\mathrm{H}_{2} \mathrm{O}}$ data with thermodynamic factors obtained from the hydration isotherm (F igure 8), are plotted in Figure 14a. There are very few direct measurements of $\tilde{D}_{\mathrm{H}_{2} \mathrm{O}}$ for water available. Frequently, pervaporation-type experiments are conducted, where the membrane is exposed to liquid water (methanol) on one side and dry flowing nitrogen on the other side.265 A chemical diffusion coefficient may be calculated from the rate of water (methanol) uptake. However, because of the extreme solvent chemical potential difference across the membrane and the thermodynamically poorly defined situation on the gas side, only average, poorly reproducible chemical diffusion coefficients are obtained; i.e., any information on the concentration dependence is lost. Another complication with these experiments is that the total pressure gradient across the membrane induces permeation, which may have contributions from additional transport mechanisms, as will be discussed later. Particularly, in the case of measuring the chemical diffusion of methanol, this problem is el egantly avoided through the use of aqueous solutions of different methanol concentration (activities) on both sides. ${ }^{266} \mathrm{M}$ any other techniques, which are frequently governed by different water (methanol) transport mechanisms (in a complex fashion), have been reviewed by Doyle and Rajendran. ${ }^{264}$

The driving force for solvent permeation is the total pressure gradient across the membrane, which is related to the chemical potential gradient, according to

$$
\nabla \vec{\mu}_{\mathrm{s}}=\left(\frac{\partial \mu_{\mathrm{s}}}{\partial \mathrm{p}}\right) \nabla \overrightarrow{\mathrm{p}}=\mathrm{V}_{\mathrm{s}}^{\mathrm{m}} \nabla \overrightarrow{\mathrm{p}}
$$

which leads to the following relation between permeation and diffusion:212

$$
\mathrm{D}_{\mathrm{s}}^{\mathrm{P}}=\frac{\mathrm{RT}}{\mathrm{C}_{\mathrm{s}}}\left(\frac{\mathrm{P}_{\mathrm{s}}}{\mathrm{V}_{\mathrm{s}}^{\mathrm{m}}}+\frac{\mathrm{K}_{\mathrm{drag}} \sigma}{\mathrm{F}^{2}}\right)
$$

where $\mathrm{P}_{\mathrm{s}}$ is the permeation coefficient and $\mathrm{K}_{\mathrm{drag}}$ is the el ectro-osmotic drag coefficient (discussed bel ow). The most comprehensive water permeation study for Nafion, as a function of water content and temperature, was reported by LaConti et al. ${ }^{267}$ and Meier et al. ${ }^{268}$ The diffusion coefficients $D_{s}^{P}$ (when adjusted to room temperature) are $\sim 1$ order of magnitude higher than the diffusion coefficients obtained by NMR techniques (Figure 14a). The "permeation diffusion coefficient" has a tendency to approach the self-diffusion coefficient only at low water contents. This behavior is not expected if the elementary mechanisms of the two types of transport are the same (i.e., quasi-random walk processes exploring the solvent chemical potential distribution in space). Obviously, there is an additional transport component in the permeation mechanism. This is most likely viscous flow far away from local thermodynamic equilibrium, which may also comprise a certain "slip" at the interface with the hydrophobic 
domain, which is a well-established phenomenon in hydrodynamics. This Hagen-Poiseuille-type component in the flow is, of course, expected to be strongly dependent on the diameter of the solvated "channels" (see Figure 13). The observation that this component apparently decreases as the water content decreases and virtually disappears in membranes with very narrow channels and strong polymer-solvent interactions (see Figure 14b and Section 4.1) is consistent with this interpretation. Hence, the mechanism of permeation in solvated acidic membranes may be described by a combination of diffusional processes and viscous flow, where the latter contribution increases as the solvent-polymer interaction decreases and the "channel" width (i.e., the degree of hydration and hydrophilic/hydrophobic separation) increases.

The aforementioned view provides a rational for the distinct differences of the solvent transport coefficients of Nafion and solvated sulfonated polyarylenes (Figure 14b), which are generally lessseparated and exhibit stronger polymer-solvent interactions.

Although solvents such as water and methanol are virtually uncharged, the application of an electrical field may induce a drift velocity of such neutral species when solvating an acidic ionomer. This phenomenon, which is known as electro-osmotic drag, is the consequence of the interaction of such solvent molecules with ions they solvate, in particular, protons in the case of PEMs. The classical mechanistic theory of electro-osmosis dates back to the time of Helmholtz, ${ }^{269}$ Lamb, ${ }^{270}$ Perrin,, 271 and Smoluchows$\mathrm{ki}, 272$ who assumed that transport occurs only close to the wall in electrical double layers of low chargecarrier concentration and with extensions significantly smaller than the pore (channel) diameter. The corresponding theories qualitatively describe the electro-osmotic drag in wide pore systems, such as clay plugs; however, as discussed in Section 3.1.2.3 (also see Figure 8), both model assumptions are not valid for typical PEM materials such as $\mathrm{N}$ afion. The width of the hydrated (solvated) channels is orders of magnitude smaller than the Debye length of water and the concentration of charge carriers is very high (typically $\sim 5 \mathrm{M}$ within the hydrophilic domain). For this type of system, Breslau and Miller developed a model for el ectro-osmosis from a hydrodynamic point of view. ${ }^{273}$ The model treats the ions as spherical particles moving in a continuous viscous medium, with the membrane matrix forming the boundary of the medium. The hydration of the ions is treated by an adequate choice of a hydrated ion radius, stripping off the hydration sphere in narrow channels, and the water structure forming and breaking properties of diverse ions are considered. Channel diameters were not directly accessible by the authors; therefore, the electro-osmotic transport of tetraalkylammoniumexchanged membranes were used to determine an "effective" pore size. Although the assumptions of the model are severe simplifications of the situation in real membranes (heterogeneity of charge and viscosity distribution (also see Figure 8) and channel widths of the extension of only a few molecules), the
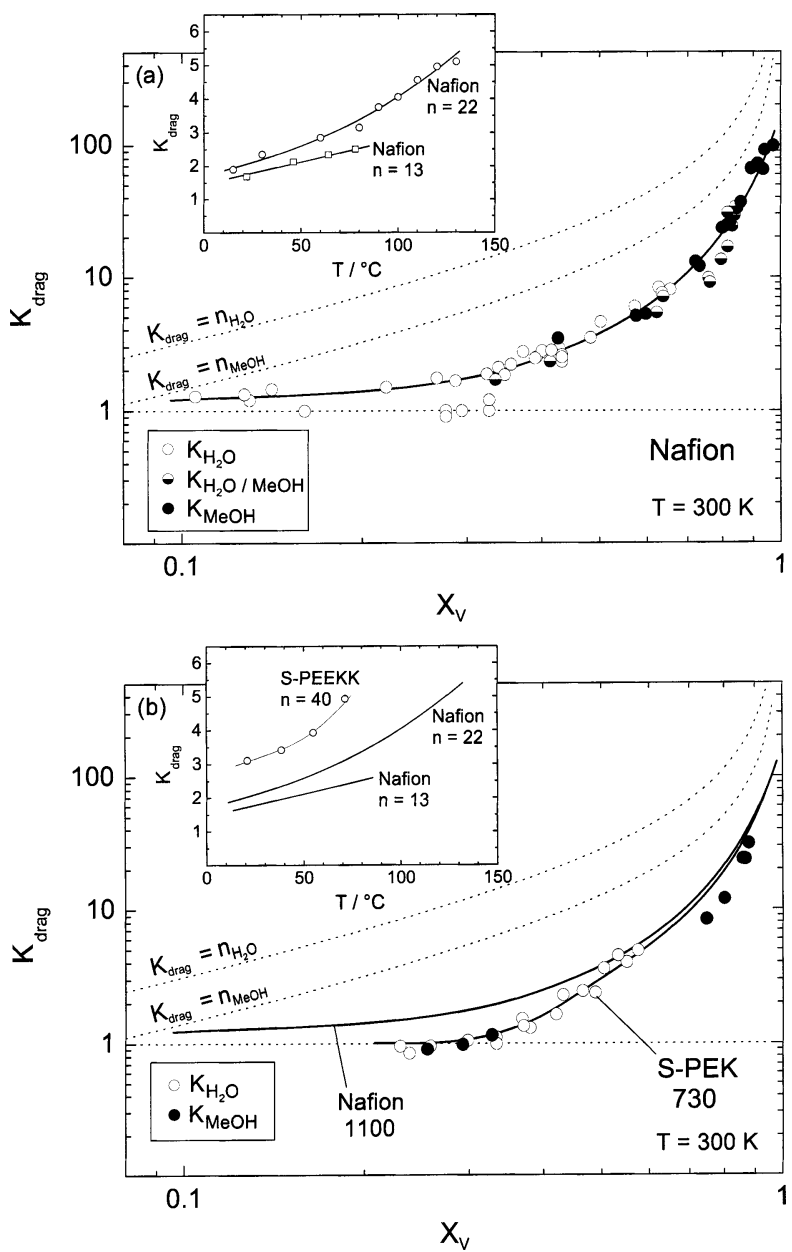

Figure 15. Electro-osmotic drag coefficients $K_{\text {drag }}$ of $(a)$ Nafion 117 (EW = 1100 g/equiv) and (b) sulfonated poly(arylene ether ketone)s, as a function of the solvent (water and/or methanol) volume fraction $X_{v}$. ${ }^{174,212,219,274-281}$ The normalized drag coefficients for water and methanol are virtually identical; therefore, both are plotted together.

model describes the variation of the electro-osmotic drag within model membranes (polystyrene-based) of different ionic forms surprisingly well.

The data were obtained for given degrees of hydration. Also, for PEM fuel-cell membranes, experimental electro-osmotic drag coefficients are generally reported only over narrow solvation ranges. Typical drag coefficients in the range of $1-2.5$ at room temperature (expressed in the number of water molecules per transported protonic charge carrier) were sometimes identified with the number of water molecules solvating the excess proton. Recently, electro-osmotic drag coefficients became accessible by electrophoretic NMR 174,212,219,274 for a wide range of polymer-solvent volume ratios, and the results clearly confirm the hydrodynamic nature of electroosmosis, particularly at high degrees of solvation. The data ${ }^{174}$ presented in Figure 15, together with data from other authors, $275-281$ essentially show two things: (i) at low degrees of hydration, the electroosmotic drag coefficient approaches a value of 1 but does not fall bel ow this value; and (ii) with increasing solvent fraction (channel width, Figure 13), the drag coefficient dramatically increases and reaches 50\% of the maximum possible value (dashed line), which corresponds to an identical drift vel ocity of all solvent 
molecules and protonic charge carriers. Considering the fact that, at high degrees of hydration, approximately half of the conductivity is carried by structure diffusion (see Section 3.1.2.1), i.e., the proton mobility (proton self-diffusion coefficient) is about twice the water self-diffusion coefficient, al most all water molecules appear to drift at approximately identical velocity (about half of the drift velocity of protonic charge carriers) in extremely swollen samples. This situation corresponds to minor relative motion of water molecules with respect to each other, i.e., the transport is clearly of a collective nature. The decrease of the drag coefficient with decreasing water content approximately scales with the fourth power of the channel diameter, which is reminescent of Hagen-Poiseuille-type behavior with continuously increasing "stripping off" of the water molecules. This stripping comes to an end at low degrees of hydration, where the motion of one water molecule remains strongly coupled to the motion of the excess proton $\left(K_{\text {drag }} \approx 1\right.$; see Figure 15$)$. This is also expected from the high enthalpy of primary hydration (stability of $\mathrm{H}_{3} \mathrm{O}^{+}$) and the proton conduction mechanism (Section 3.2.1.2), i.e., diffusion of $\mathrm{H}_{3} \mathrm{O}^{+}$(vehicle mechanism).

Extremely high degrees of swelling were actually obtained in water/methanol mixtures; and the drag coefficients of water and methanol were determined separately by isotopic labeling (H/D substitution). Interestingly, the normalized drag coefficients (drag coefficient $\mathrm{K}_{\mathrm{i}}$ divided by the mole fraction of the corresponding species i) are virtually identical for both species in the mixtures (also see Figure 15). Because water and methanol form almost-ideal solutions with comparable water-water, water-methanol, and methanol-methanol interactions, this behavior provides additional support for a hydrodynamic process. For low degrees of hydration, where electroosmotic drag becomes diffusional in nature, it is not yet clear, whether there is some preferential solvation of the excess proton by water or methanol (primary hydration). Figure 15b shows that the electro-osmotic drag for sulfonated poly(arylene ether ketone)s is generally lower than that for Nafion, which is partly due to smaller channels. However, even for comparable channel dimensions, the values for electroosmotic drag are still somewhat lower, which is an indication of the influence of the solvent-polymer interaction on the total el ectro-osmotic drag. This is also supported by the observation that electro-osmotic drag is generally increasing with increasing temperature (see inserts in Figures $15 a$ and b), whereas the solvent-polymer interaction is decreasing.

There is no quantitative model yet describing the observed electro-osmotic drag coefficients as a function of the degree of hydration and temperature. However, the available data provide strong evidence for a mechanism that is (i) hydrodynamic in the high solvation limit, with the dimensions of the solvated hydrophilic domain and the solvent-polymer interaction as the major parameters; and (ii) diffusive at low degrees of solvation, where the excess proton essentially drags its primary solvation shell (e.g., $\mathrm{H}_{3} \mathrm{O}^{+}$).
3.2.1.2. Mechanisms of Dissolved Gas Transport. As noted in Section 1, one of the cardinal properties of fuel-cell membrane materials is to be able to effectively separate the reactive masses, in particular, the anode and cathode gases. Accordingly, there have been numerous investigations, especially on oxygen and hydrogen transport across PEMs, and because the current qualitative mechanistic understanding has already existed for some time, only a brief summary is given here.

It already had been recognized by $\mathrm{Yeo}$ and McBreen 282 that gas (hydrogen and halogens) permeation rates in Nafion are strongly related to water content. The data indicated that most of the gas transport is occurring within the solvated hydrophilic domain at rates only slightly lower than these of water self-diffusion. Ogumi and co-workers 283,284 later reported that oxygen diffusion in different hydrated membranes are indeed very similar, with the gas solubilities differing significantly. These results made al ready clear that gases dissolve in both the hydrophobic and the solvated hydrophilic domain, whereas most of the gas transport occurs within the solvated hydrophilic domain. This view is supported by many experimental studies on Nafion ${ }^{285-287}$ and other types of membranes. ${ }^{288,289}$ When hydrated, they all show comparable diffusion and permeation rates for oxygen and hydrogen, and it was not surprising that significantly lower gas transport rates were found for Nafion when water was replaced by phosphoric acid $^{290}$ or when Nafion was dried. ${ }^{291}$ In the latter case, the gas diffusion rates were reported to be similar to those of plain polytetrafluoroethane (PTFE). Büchi et al..$^{292}$ actually separated the oxygen solubility and diffusion as a function of temperature for different perfluorinated proton exchange membranes of different equivalent weights. Apart from giving further support to the existing mechanistic understanding, this work showed that the oxygen solubility is decreasing while oxygen permeation is increasing with temperature.

\subsubsection{Oxides}

From the formation reaction of protonic defects in oxides (eq 23), it is evident that protonic defects coexist with oxide ion vacancies, where the ratio of their concentrations is dependent on temperature and water partial pressure. The formation of protonic defects actually requires the uptake of water from the environment and the transport of water within the oxide lattice. Of course, water does not diffuse as such, but rather, as a result of the ambipolar diffusion of protonic defects $\left(\mathrm{OH}_{\mathrm{O}}^{\circ}\right)$ and oxide ion vacancies $\left(\mathrm{V}_{\mathrm{O}}^{\circ}\right)$. Assuming ideal behavior of the involved defects (an activity coefficient of unity) the chemical (Fick's) diffusion coefficient of water is

$$
\tilde{D}_{\mathrm{H}_{2} \mathrm{O}}=\frac{(2-X) \mathrm{D}_{\mathrm{OH}_{\mathrm{O}}^{\cdot}} \mathrm{D}_{\mathrm{V}_{\mathrm{O}}^{*}}}{X D_{\mathrm{OH}_{\mathrm{O}}^{*}}+2(1-X) \mathrm{D}_{\mathrm{V}_{\mathrm{o}}}}
$$

where $X$ is the degree of hydration. ${ }^{293}$ Under completely dry conditions, i.e., in the absence of proton conductivity, $\tilde{\mathrm{D}}_{2} \mathrm{O}$ equals the self-diffusion coefficient 


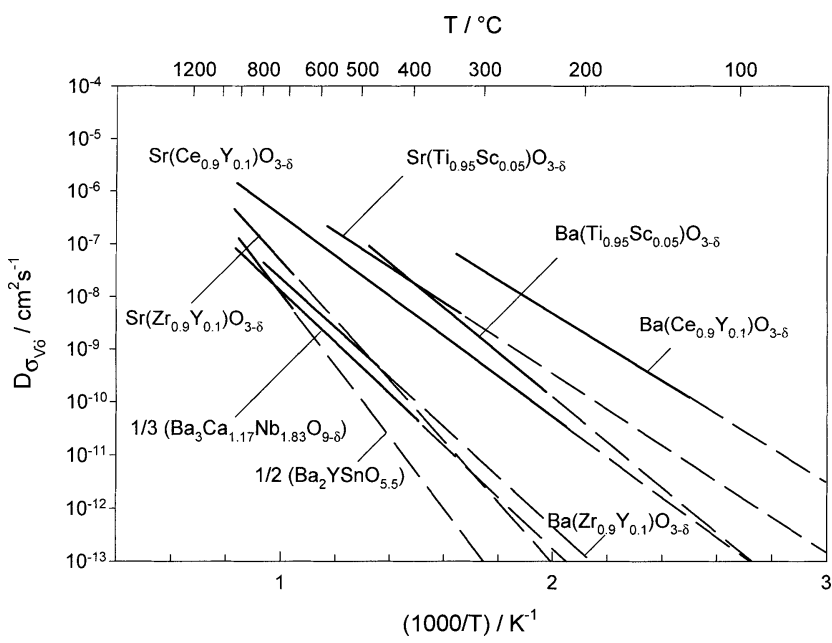

Figure 16. Self-diffusion coefficient of oxide ion vacancies in different perovskite-type oxides, ${ }^{188}$ which equals the chemical water diffusion coefficient in the fully hydrated state (see text). (Figure reproduced with the kind permission from Elsevier.)

of protonic defects. However, near the hydration limit (i.e., $\mathrm{X}$ close to 1 ), $\tilde{\mathrm{D}}_{\mathrm{H}_{2} \mathrm{O}}$ approaches the self-diffusion coefficient of oxide ion vacancies, which is usually significantly lower than the diffusion coefficient of protonic defects. Because the latter case relates to the conditions in a fuel cell, the self-diffusion coefficient of oxide ion vacancies, which is shown in Figure 16 for a variety of perovskite-type oxides, may be taken as a measure of $\mathrm{D}_{\mathrm{H}_{2} \mathrm{O}}$ under these conditions. For a given water partial pressure, $\tilde{\mathrm{D}}_{\mathrm{H}_{2} \mathrm{O}}$ increases as temperature increases, because of dehydration and the increasing self-diffusion coefficients for both involved species. Recently, there has been some controversy about the validity of the model; 294 however, it is indeed strictly valid in the entire hydration range under the given assumptions. ${ }^{295}$

It should be mentioned that, apart from oxide ion vacancies existing at low water partial pressures, the appearance of electronic holes $\left(h^{\circ}\right)$ must be considered at high oxygen activities. In oxides, they are frequently localized on the oxygen $\left(\mathrm{O}^{2-}+\mathrm{h}^{\bullet} \rightleftharpoons \mathrm{O}^{-}\right)$and may be very mobile as large polarons. The ambi polar diffusion, together with protonic defects, then permits chemical diffusion of hydrogen; and the ambipolar diffusion, together with oxide ion vacancies, allows chemical diffusion of oxygen. However, for largeband-gap oxides relevant for fuel-cell applications, this may not be important at temperatures $<750$ ${ }^{\circ} \mathrm{C}$. ${ }^{296-298}$

\section{Phenomenology of Transport in Proton-Conducting Materials for Fuel-Cell Applications}

Transport data have been reported for a huge number of proton-conducting materials, some of which are defined rather poorly. They have been recorded by a variety of methods under diverse conditions including ill-defined transient or steadystate situations (e.g., in a running fuel cell). Consequently, reproducibility and comparison of data from different laboratories is problematic. F or this reason, we have only compiled the transport data that have been obtained under thermodynamically defined conditions, preferably those close to thermodynamic equilibrium, i.e., with relatively small perturbations (electrical potential gradients, chemical potential gradients, etc.) applied to the samples. Although far from complete, the diversity of the materials included in this review allow for some general qualitative conclusions (see Section 5). The transport coefficients presented may also allow calculation (or numerical simulation) of fluxes and concentrations for nonequilibrium situations, provided that linear response may be assumed. The purpose of this section is to summarize the transport coefficients of some typical and well-characterized proton conductors without giving any mechanistic explanations, which al ready have been discussed in Section 3 for prototypical compounds, and also provide rational for a qualitative understanding of the phenomenological data presented in this section.

\subsection{Hydrated Acidic Polymers}

For the application of acidic polymers in fuel cells, the most relevant species, for which transport must be considered, are protonic charge carriers, water, methanol, hydrogen, and oxygen. As discussed in Section 3, the transport of all these species predominantly occurs in the same environment, i.e., the solvated hydrophilic domain of the material. In addition to the transport coefficients for each of the five species, one must al so consider cross coefficients describing the coupled transport of different species. For a linear description, this al ready requires a $5 \times$ 5 matrix with 15 independent coefficients. Together with the publications referenced in Section 3.2.1.2, two more recent studies 299,300 provide data and further literature on gas (predominately $\mathrm{O}_{2}$ and $\mathrm{H}_{2}$ ) transport in solvated acidic membranes. The reproducibility of these data is somewhat less than that for water transport; however, the general behavior is such that $\mathrm{O}_{2}$ diffusion is closely related to water diffusion, including its dependence on solvation and temperature, whereas the $\mathrm{H}_{2}$ diffusion is generally higher (by factors of 2-4). Because the gas solubility within the hydrophilic domain is quite low (i.e., on the order of $10^{-6} \mathrm{~mol} \mathrm{~cm}^{-3}$ for $\mathrm{O}_{2}$ within the hydrated domain of Nafion at ambient conditions) and decreases with temperature, the gas permeability remains quite low (for $\mathrm{O}_{2}$ on the order of $10^{-11} \mathrm{~mol} \mathrm{~cm}^{-1}$ $\mathrm{s}^{-1}$ in Nafion) and does not increase with temperature to the same degree as the gas and solvent diffusion coefficients.

The following compilation is restricted to the transport coefficients of protonic charge carriers, water, and methanol. These may be represented by a $3 \times 3$ matrix with six independent el ements if it is assumed that there is just one mechanism for the transport of each species and their couplings. However, as discussed in Sections 3.1.2.1 and 3.2.1, different types of transport occur, i.e., diffusive transport as usually observed in the solid state and additional hydrodynamic transport (viscous flow), especially at high degrees of solvation. Assuming that the total fluxes are simply the sum of diffusive and hydrodynamic components, the transport matrix may 
be extended by three more phenomenological elements describing (i) the hydrodynamic components of proton transport coupled to water or methanol permeation, and (ii) the water, and methanol, permeations themselves. Of course, the description of transport with a $3 \times 4$ matrix is neither complete nor consistent; however, it is a comfortable way to describe transport in solvated acidic membranes phenomenologically with reasonable precision. The transport equation may then be written

$$
\begin{array}{r}
\left(\begin{array}{l}
\vec{\jmath}_{\mathrm{H}^{+}} \\
\vec{\jmath}_{\mathrm{H}_{2} \mathrm{O}} \\
\vec{\jmath}_{\mathrm{MeOH}}
\end{array}\right)=\left(\begin{array}{llll}
\mathrm{L}_{11} & \mathrm{~L}_{12} & \mathrm{~L}_{13} & \mathrm{~L}_{14} \\
\mathrm{~L}_{21} & \mathrm{~L}_{22} & \mathrm{~L}_{23} & \mathrm{~L}_{24} \\
\mathrm{~L}_{31} & \mathrm{~L}_{32} & \mathrm{~L}_{33} & \mathrm{~L}_{34}
\end{array}\right)\left(\begin{array}{l}
\nabla \tilde{\mu}_{\mathrm{H}^{+}} \\
\nabla \vec{\mu}_{\mathrm{H}_{2} \mathrm{O}} \\
\nabla \vec{\mu}_{\mathrm{MeOH}} \\
\nabla \vec{p}_{\text {total }}
\end{array}\right) \\
\text { with } \mathrm{L}_{\mathrm{ij}}=\mathrm{L}_{\mathrm{ji}}
\end{array}
$$

with the driving forces being the gradients of

$$
\begin{aligned}
& \tilde{\mu}_{\mathrm{H}^{+}}=\mu_{\mathrm{H}^{+}}^{0}+\mathrm{RT} \text { In } \mathrm{a}_{\mathrm{H}^{+}}+\mathrm{V}_{\mathrm{H}^{+}}^{\mathrm{m}} \mathrm{p}_{\text {total }}+\mathrm{z}_{\mathrm{H}^{+}} \mathrm{F} \Phi \\
& \mu_{\mathrm{H}_{2} \mathrm{O}}=\mu_{\mathrm{H}_{2} \mathrm{O}}^{0}+\mathrm{RT} \text { In } \mathrm{a}_{\mathrm{H}_{2} \mathrm{O}}+\mathrm{V}_{\mathrm{H}_{2} \mathrm{O}}^{\mathrm{m}} \mathrm{p}_{\text {total }} \\
& \mu_{\mathrm{MeOH}}=\mu_{\mathrm{MeOH}}^{0}+\mathrm{RT} \text { In } \mathrm{a}_{\mathrm{MeOH}}+\mathrm{V}_{\mathrm{MeOH}}^{\mathrm{m}} \mathrm{p}_{\text {total }}
\end{aligned}
$$

The longitudinal coefficient $L_{11}$ is related to the proton conductivity $\sigma$, according to

$$
\mathrm{L}_{11}=\frac{\sigma}{\mathrm{F}^{2}}
$$

$L_{22}$ and $L_{33}$ are related to the self-diffusion and chemical diffusion coefficients of water and methanol:

$$
\begin{gathered}
L_{22}=\frac{C_{\mathrm{H}_{2} \mathrm{O}}^{2}}{R T}\left(\frac{d\left(I n a_{\mathrm{H}_{2} \mathrm{O}}\right)}{d c_{\mathrm{H}_{2} \mathrm{O}}}\right) D_{\mathrm{H}_{2} \mathrm{O}}=\frac{C_{\mathrm{H}_{2} \mathrm{O}}}{R T} \tilde{D}_{\mathrm{H}_{2} \mathrm{O}} \\
\mathrm{L}_{33}=\frac{\mathrm{C}_{\mathrm{MeOH}}^{2}}{R T}\left(\frac{d\left(\ln \mathrm{a}_{\mathrm{MeOH}}\right)}{d c_{\mathrm{MeOH}}}\right) D_{\mathrm{MeOH}}=\frac{C_{\mathrm{MeOH}}}{R T} \tilde{D}_{\mathrm{MeOH}}
\end{gathered}
$$

The cross coefficients are contained in the electroosmotic drag coefficients:

$$
\begin{gathered}
\mathrm{K}_{\mathrm{H}_{2} \mathrm{O}}=\frac{\mathrm{L}_{21}}{\mathrm{~L}_{11}} \\
\mathrm{~K}_{\mathrm{MeOH}}=\frac{\mathrm{L}_{31}}{\mathrm{~L}_{11}}
\end{gathered}
$$

The coefficients $L_{14}, L_{24}$, and $L_{34}$ describe the viscous flow contributions of the transport of all three species in a total pressure gradient $\nabla \vec{p}_{\text {total }}$. Because a pressure gradient also imposes a chemical potential gradient on each species (eq 24), experimentally, there is always a superposition of diffusive and viscous flow; e.g., for the description of the water flux in a total pressure gradient, all coefficients must be included, i.e.,

$$
\begin{array}{r}
\vec{J}_{\mathrm{H}_{2} \mathrm{O}}=\mathrm{L}_{21} \nabla \tilde{\mu}_{\mathrm{H}^{+}}+\mathrm{L}_{22} \nabla \vec{\mu}_{\mathrm{H}_{2} \mathrm{O}}+\mathrm{L}_{23} \nabla \vec{\mu}_{\mathrm{MeOH}}+ \\
\mathrm{L}_{24} \nabla \vec{p}_{\text {total }}=\mathrm{P}_{\mathrm{H}_{2} \mathrm{O}} \nabla \vec{p}_{\text {total }}
\end{array}
$$

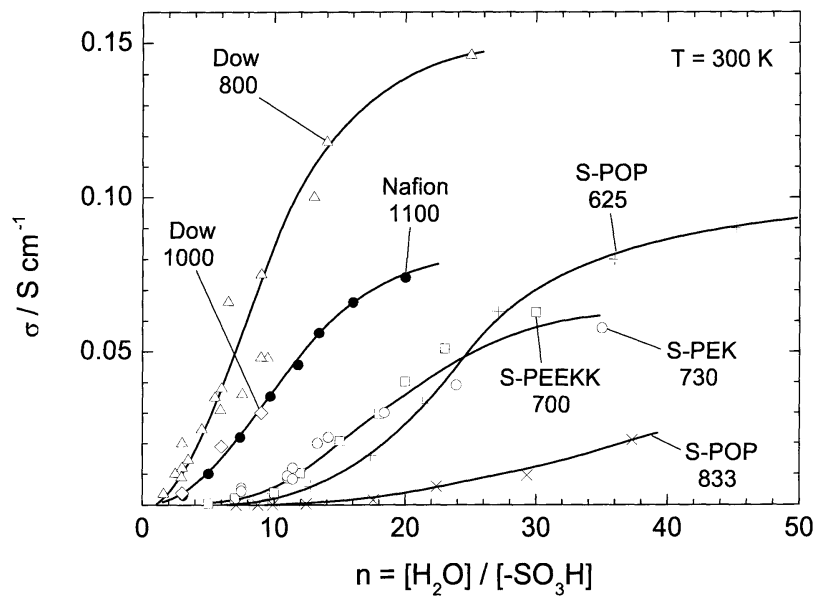

Figure 17. Room-temperature proton conductivity of two Dow membranes $226,255,260$ of different EW values, Nafion, two varieties of sulfonated poly(arylene ether ketone)s (SPEK and S-PEEKK, unpublished data from the laboratory of one of the authors), and sulfonated poly(phenoxyphosphazene)s (S-POPs ${ }^{301}$ ) of different equivalent weights (685 and $833 \mathrm{~g}$ (equiv), as a function of the degree of hydration $\mathrm{n}=\left[\mathrm{H}_{2} \mathrm{O}\right] /\left[-\mathrm{SO}_{3} \mathrm{H}\right]$ (number below the compound acronym/ name indicates the EW value).

Usually, the total permeation coefficient $\left(\mathrm{P}_{\mathrm{H}_{2} \mathrm{O}}\right)$ is measured, and this, expressed as a permeation diffusion coefficient $D_{\mathrm{H}_{2} \mathrm{O}}^{P}$, is given in the following data compilation.

There are actually no experimental measurements of protonic streaming currents $\left(\mathrm{L}_{14}\right)$ and coupled water and methanol transport $\left(\mathrm{L}_{23}=\mathrm{L}_{32}\right)$; however, the first may be related to the hydrodynamic component of the electro-osmotic drag $\left(\mathrm{L}_{12} / \mathrm{L}_{11}, \mathrm{~L}_{13} / \mathrm{L}_{11}\right)$ (see discussion in Section 3.2.1.1). The second is expected to be qualitatively related to the ratio of the electro-osmotic drag coefficient of water and methanol $\left(\mathrm{L}_{12} / \mathrm{L}_{13}\right)$. In the following, the directly accessible transport coefficients $\left(\sigma\left(\mathrm{D}_{\sigma}\right), \mathrm{D}_{\mathrm{H}_{2} \mathrm{O}}, \mathrm{D}_{\mathrm{MeOH}}, \mathrm{D}_{\mathrm{H}_{2} \mathrm{O}}^{\mathrm{P}}\right.$, $\mathrm{D}_{\mathrm{MeOH}}^{\mathrm{P}}, \mathrm{K}_{\mathrm{H}_{2} \mathrm{O}}$, and $\mathrm{K}_{\mathrm{MeOH}}$ ) for different solvated acidic polymers are presented in a way that allows some interesting comparisons and the calculation or estimation of the elements of the transport matrix $L_{i j}$. In many publications, these transport parameters are reported as a function of the solvent content and are expressed as the number of solvent molecules (i.e., water) per sulfonic acid group. Because of the importance of percolation effects in all considered transport coefficients, we have converted these solvent contents to solvent volume fractions, except for proton conductivities, as shown in Figures 17 and 18.

Figure 17 shows the room-temperature proton conductivity of two sulfonated poly(arylene ether ketone)s, sulfonated poly(phenoxyphosphazene)s (SPOP) of different equivalent weights, ${ }^{301-303} \mathrm{~N}$ afion (unpublished data from the laboratory of one of the authors), and two Dow membranes of different equivalent weight. ${ }^{226,255,260}$ Except for one Dow membrane with an equivalent weight of 800 g/equiv, the other membranes have similar (volume) densities of sulfonic acid groups, i.e., similar water volume fractions for a given water content $n=\left[\mathrm{H}_{2} \mathrm{O}\right] /\left[-\mathrm{SO}_{3} \mathrm{H}\right]$. It is a general observation that hydrocarbon membranes require more water to achieve similar conductivities than perfluorosulfonic acid polymers. This effect is 
$\mathrm{T} /{ }^{\circ} \mathrm{C}$

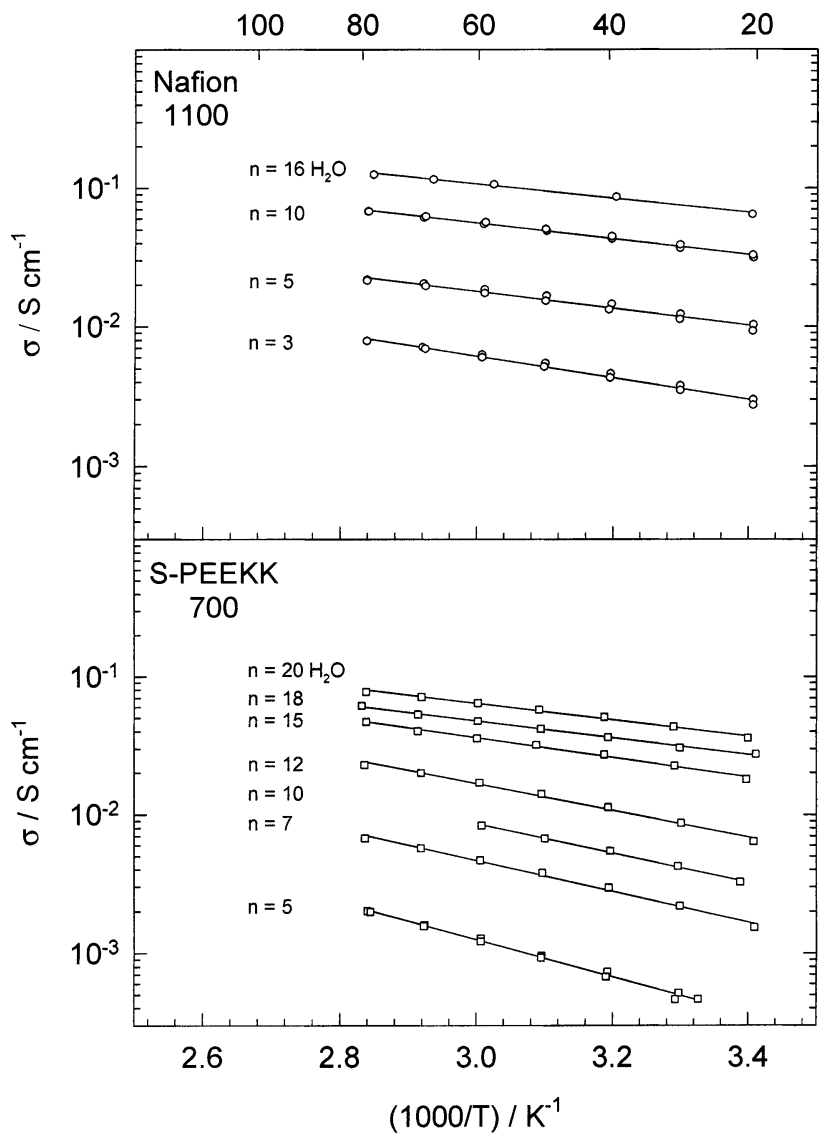

Figure 18. Proton conductivity of (a) Nafion 117 (EW = $1100 \mathrm{~g} /$ equiv) and (b) a sulfonated poly(arylene ether ketone), as a function of temperature and degree of hydration $\left(n=\left[\mathrm{H}_{2} \mathrm{O}\right] /\left[-\mathrm{SO}_{3} \mathrm{H}\right]\right){ }^{197}$

most pronounced for the high-equivalent-weight S-POP 833, and, considering the relation between solvent diffusion and proton mobility, this observation is in accordance with the low solvent diffusion coefficients reported for this type of membrane. ${ }^{302}$ To reach sufficiently high conductivities for fuel-cell applications, such membranes must be in contact with liquid water. ${ }^{219}$

Figure 18 shows the temperature dependence of the proton conductivity of Nafion and one variety of a sulfonated poly(arylene ether ketone) (unpublished data from the laboratory of one of the authors). The transport properties of the two materials are typical for these classes of membrane materials, based on perfluorinated and hydrocarbon polymers. This is clear from a compilation of $\mathrm{D}_{\sigma}, \mathrm{D}_{\mathrm{H}_{2} \mathrm{O}}$, and $\mathrm{D}_{\mathrm{H}_{2} \mathrm{O}}^{\mathrm{O}}$ data for a variety of membrane materials, including Dow membranes of different equivalent weights, 226,255,260 $\mathrm{Nafion} / \mathrm{SiO}_{2}$ composites 243,244,304-306 (including unpublished data from the laboratory of one of the authors), cross-linked polyarylenes, ${ }^{307-315}$ and sulfonated poly(phenoxyphosphazenes) ${ }^{301}$ (Figure 19). The data points all center around the curves for Nafion and S-PEK, indicating essentially universal transport behavior for the two classes of membrane materials (only for S-POP are the transport coefficients somewhat lower, suggesting a more reduced percolation in this particular material). This correlation is also true for the electro-osmotic drag coefficients $\mathrm{K}_{\mathrm{H}_{2} \mathrm{O}}$ and $\mathrm{K}_{\mathrm{MeOH}}$

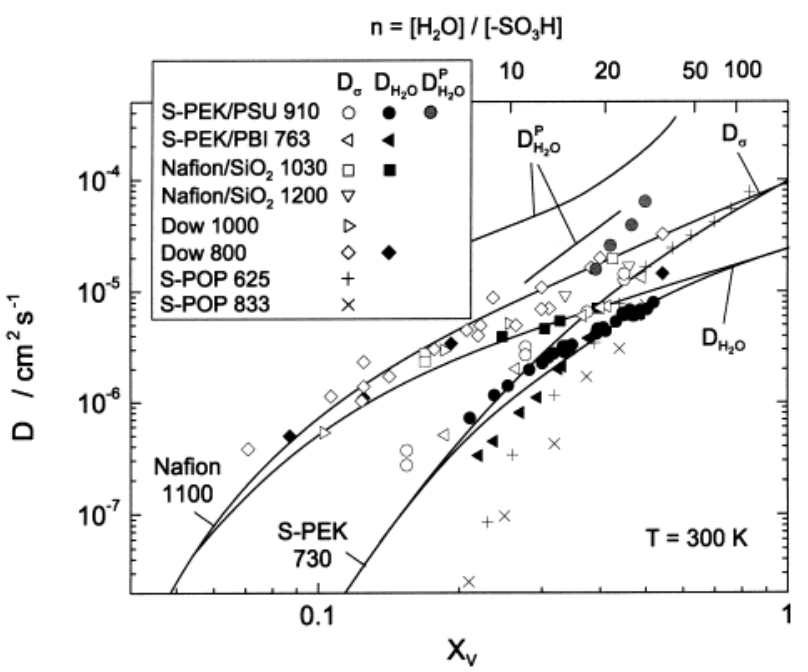

Figure 19. Transport coefficients of diverse membranes based on perfluorinated polymers (Dow 226,255,260 and Nafion/ silica composites ${ }^{174,243,244,304-306}$ ), polyarylenes (S-PEK/PSU blends, ionically cross-linked S-PEK/PBI ), $307-315$ and sulfonated poly(phenoxyphosphazene)s (S-POPs), ${ }^{301}$ as a function of the water volume fraction $X_{v}$. Lines represent data for Nafion and S-PEK (given for comparison); for data points, see Figure 14

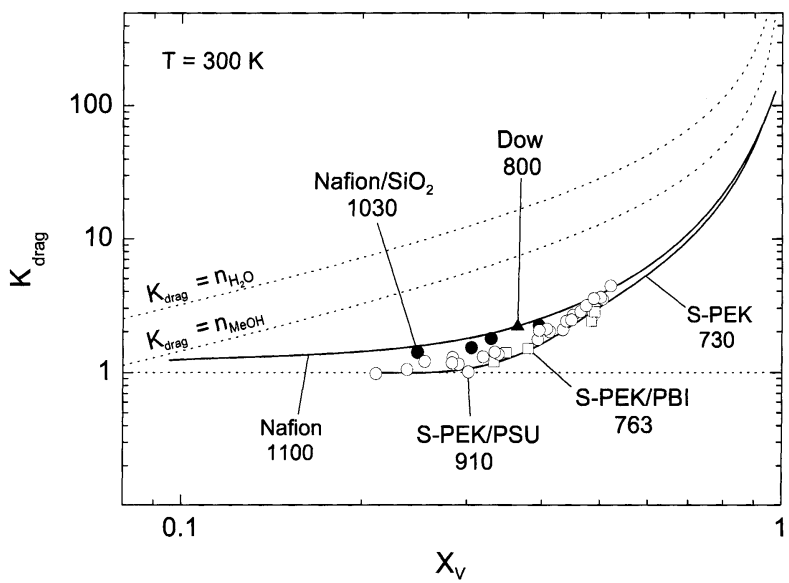

Figure 20. Electro-osmotic drag coefficients of diverse membranes based on perfluorinated polymers (Dow ${ }^{226,276}$ and Nafion/silica composites ${ }^{174}$ ) and polyarylenes (S-PEK/ PSU blends, ionically cross-linked S-PEK/PBI ${ }^{174}$ ), as a function of the solvent (water/methanol) volume fraction $X_{V}$ (see text for references). Lines represent data for N afion and S-PEK (given for comparison); for data points, see Figure 15. Dashed lines correspond to the maximum possible electro-osmotic drag coefficients for water and methanol, as indicated (see text).

(Figure 20; for this figure, data for the Dow membrane are taken from refs 226 and 276; all other data are from the laboratory of one of the authors), which suggests that the major parameter that controls electro-osmotic transport is simply the degree of swelling and the choice of the type of backbone, particularly for low degrees of swelling (perfluorinated versus hydrocarbon polymer).

\section{2. $\mathrm{PBI}-\mathrm{H}_{3} \mathrm{PO}_{4}$ Adducts}

The transport data of $\mathrm{PBI}-\mathrm{H}_{3} \mathrm{PO}_{4}$ have extensively been reviewed in two recent papers, ${ }^{316,317}$ and their hybrids with inorganic particles have been characterized in another. ${ }^{318}$ The gas permeability is much 


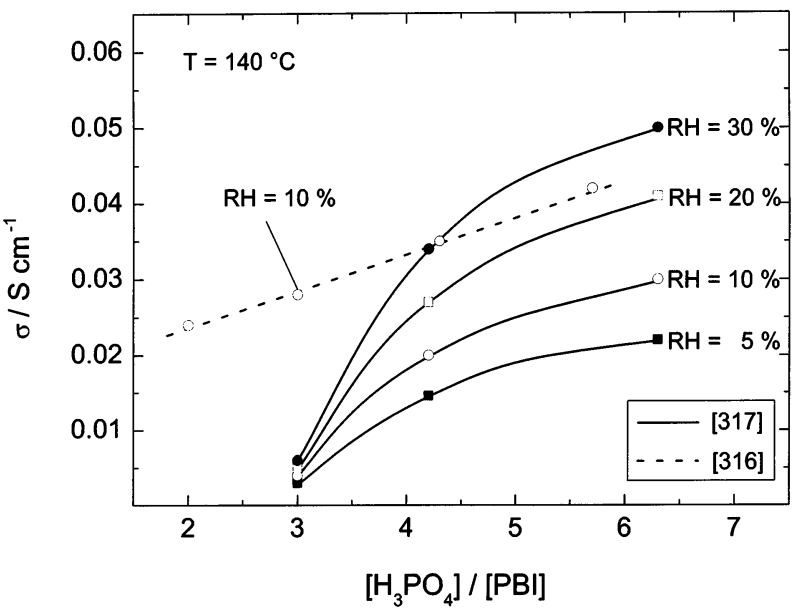

Figure 21. Proton conductivity of $\mathrm{PBI} \cdot \mathrm{nH}_{3} \mathrm{PO}_{4}$ adducts, as a function of phosphoric acid concentration and relative humidity (RH). ${ }^{317}$ Data from another source (denoted by the dashed line) ${ }^{316}$ are given for comparison.

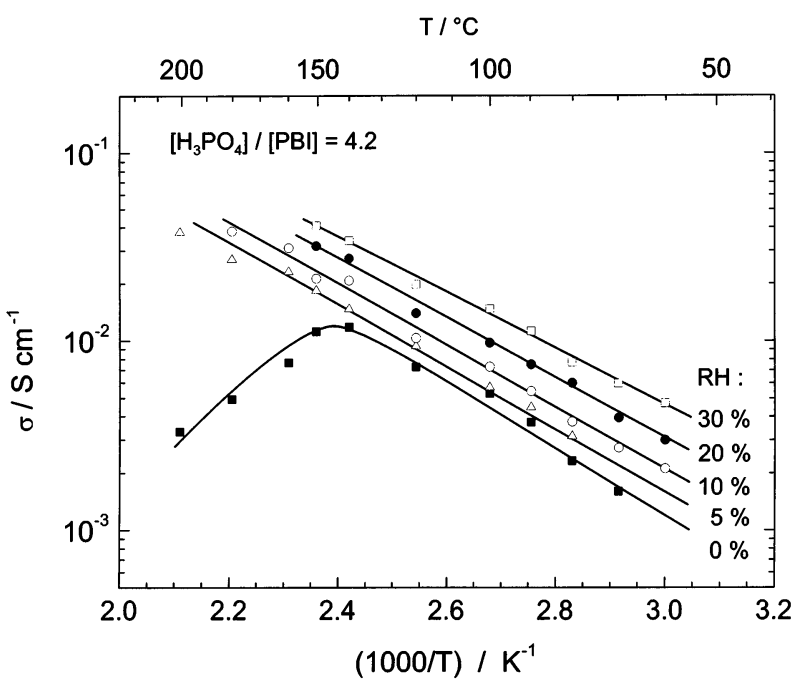

Figure 22. Proton conductivity of $\mathrm{PBI} \cdot \mathrm{nH}_{3} \mathrm{PO}_{4}$ adducts, as a function of temperature $T$ and relative humidity $\mathrm{RH}$ for a given phosphoric acid concentration. ${ }^{317}$

lower than that in hydrated acidic polymers, and there is almost no water electro-osmotic drag, even at high relative humidities. ${ }^{254}$ The proton conductivity is mainly dependent on the fraction of phosphoric acid (frequently termed "doping level"), relative humidity $(\mathrm{RH})$, and temperature. The corresponding dependencies are shown in Figures 21 and 22. The data suggest that the presence of water has two effects on the conductivity: (i) a minimum RH value is required to prevent the phosphoric acid from condensing at temperatures above $\mathrm{T} \approx 100{ }^{\circ} \mathrm{C}$; and (ii) excess water leads to a further increase of the conductivity, as observed in pure phosphoric acid (Section 3.1.1.2). The data in Figures 21 and 22 are actually taken from one reference, ${ }^{317}$ except for one curve taken from ref 316, which has been included to demonstrate the only moderate reproducibility.

\subsection{Heterocycle-Based Systems}

The first heterocycle-based systems that had some relevance in the development of new types of fuelcell membranes were sulfonated polyarylenes ${ }^{170}$ and
Nafion ${ }^{234}$ with intercalated heterocycles. Conductivities of $>10^{-2} \mathrm{~S} / \mathrm{cm}$ are easily accessible by such systems; however, the volatility of the heterocycles requires some immobilization. The effect of covalent immobilization on proton conductivity is shown in Figure 23 for imidazole-based systems. ${ }^{213,237-239,241,242,319}$ It is important to realize that these oligomers have similarly low vapor pressures as ionic liquids. To illustrate the effect of extrinsic charge carrier formation, the evolution of proton conductivity with triflic acid doping is shown for the ol igomeric system I mi-2 in Figure $24 .{ }^{237} \mathrm{~F}$ or such systems, the mobility of protonic charge carriers is typically 1 order of magnitude higher than the self-diffusion of the oligomers as a whole. Of course, long-range solvent (imidazole, pyrazole, benzimidazole) transport does not occur in fully polymeric systems. Cyclovol tammetry and fuelcell-type experiments demonstrate that there is at least some transport of oxygen and hydrogen in imidazole-based systems. ${ }^{320}$

\subsection{Oxides}

Apart form protonic defects, the only species that may betransported in proton-conducting wide-bandgap perovskite-type oxides at significant rates are oxide ion vacancies. Although this allows for some chemical water diffusion in the intermediate temperature range (typically $300-700{ }^{\circ} \mathrm{C}$, see Section 3.2.2), hydrogen and oxygen diffusion only occurs under either highly reducing or oxidizing conditions and at significantly higher temperatures, when electrons and hol es become relevant as additional defects. Therefore, only proton conductivities for a variety of oxides at a water partial pressure of $\mathrm{p}_{\mathrm{H}_{2} \mathrm{O}}=30 \mathrm{hPa}$ are shown in Figure 25 (ref 186 with data from ref 187). Note that the dopant concentration for the examples shown is $~ 10 \%$ and that the proton mobility in such samples may significantly deviate from this in undoped materials (see Section 3.1.1.4).

\section{Recent Approaches toward New Proton-Conducting Materials for Fuel-Cell Applications}

The suitability of proton-conducting materials as separators in a particular fuel-cell application is essentially dependent on its transport properties, durability, and reactivity. Thus far, this review has focused on the transport properties only, but any approach toward new separator materials must consider all relevant aspects, which makes the development of new competitive materials a complex and challenging task.

Hence, most current strategies are trying to extend the application limits while conserving the specific advantages of well-established sulfonated materials such as Nafion by slightly varying or modifying them. Apart from the maximum operating temperature and the high crossover of water and methanol, long-term stability under fuel-cell conditions is also a severe problem. The first two constraints are related to the humidification requirements (see Section 1 ), and most materials development processes end up compromising on high proton conductivity on one side 


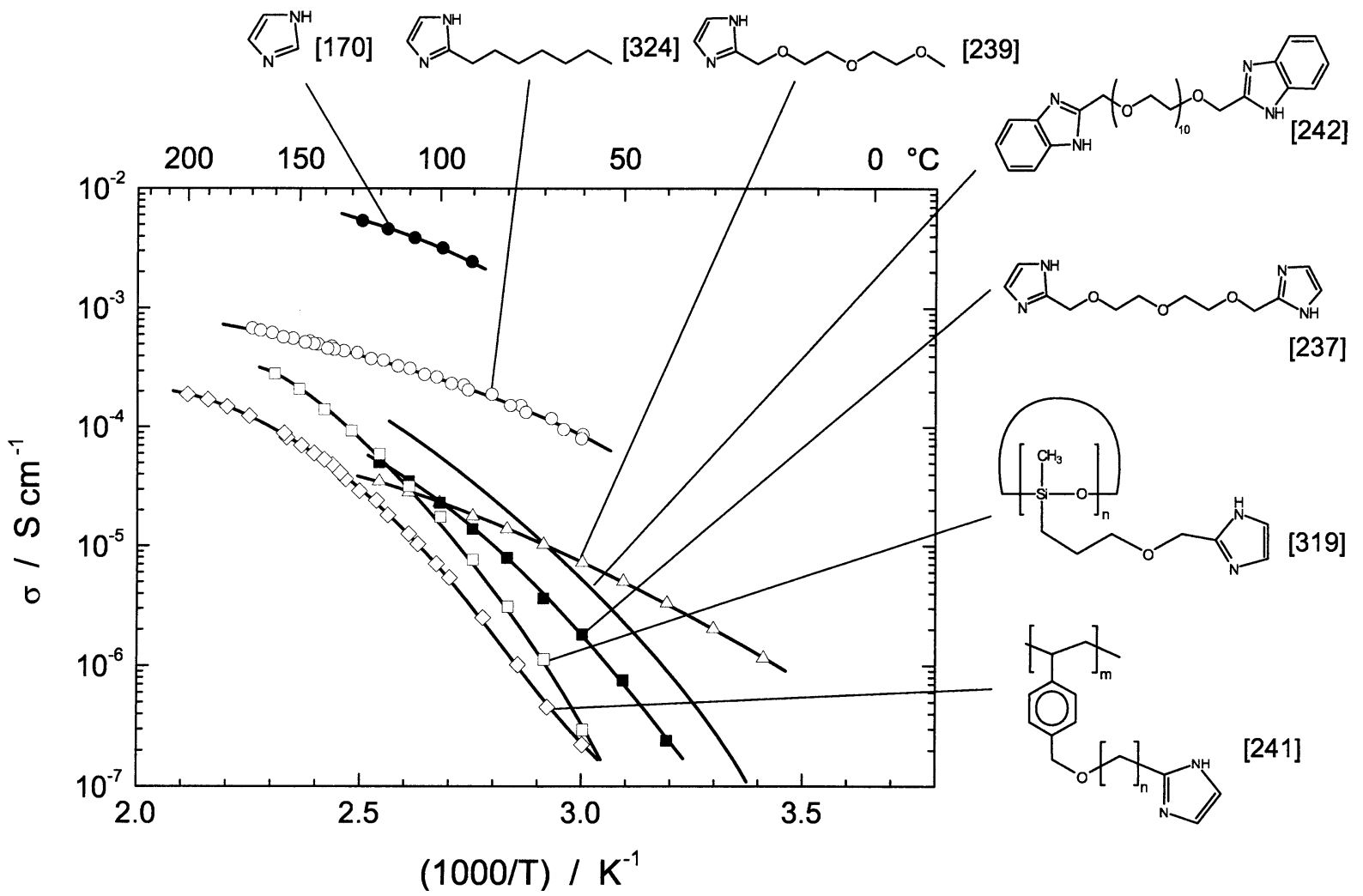

Figure 23. Evolution of proton conductivity of imidazol e-based systems with increasing immobilization: from the monomer via oligomers to fully polymeric systems.

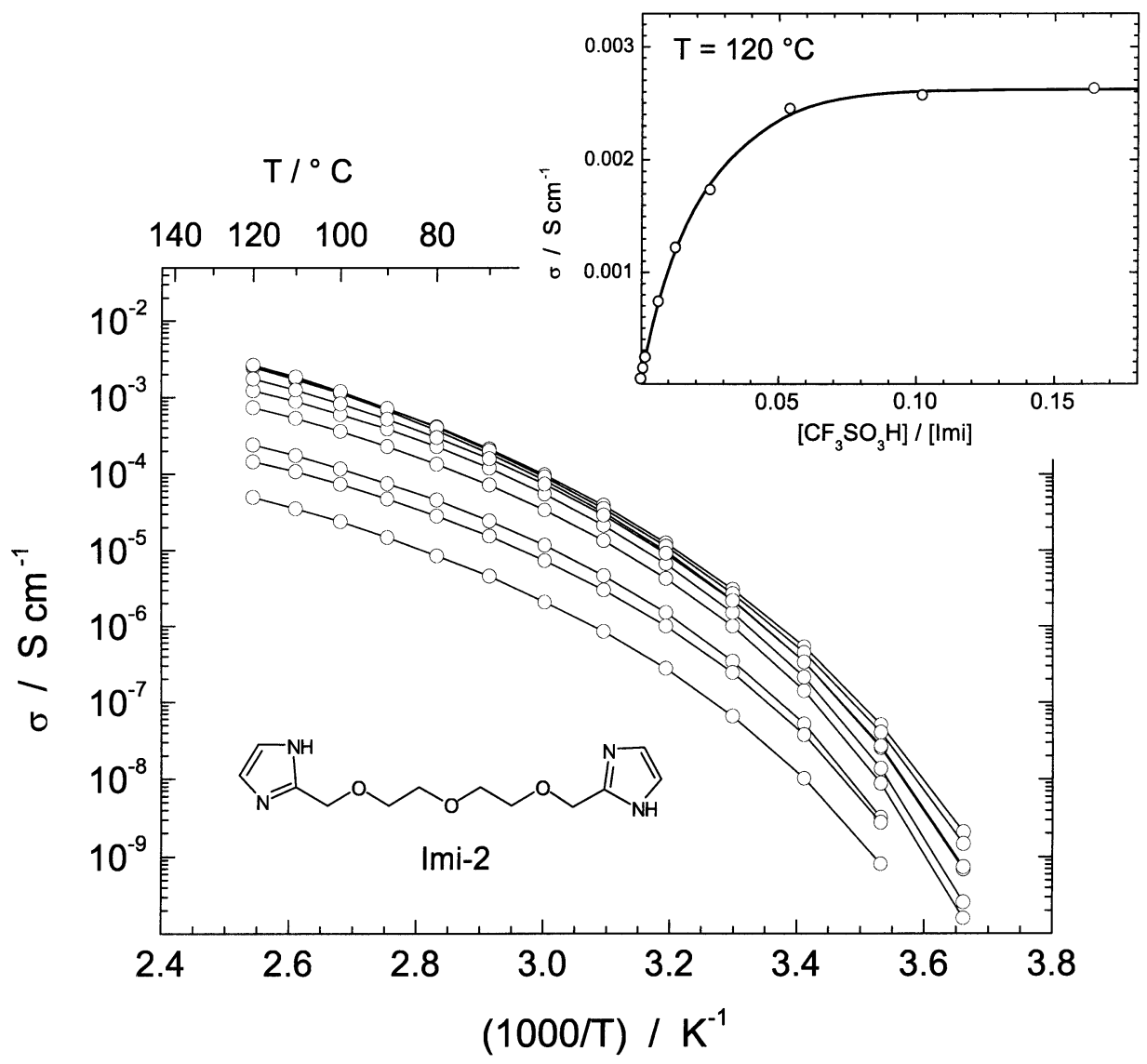

Figure 24. Proton conductivity of Imi-2 (two imidazoles spaced by two ethylene oxide (EO) units), as a function of triflic acid doping. ${ }^{237}$ Note that the conductivity has a tendency to level off at high acid concentration (see insert).

and morphological stability (low swelling and insolubility at elevated temperature) and low water and methanol crossover on the other side. One crucial parameter that allows control of these properties is 


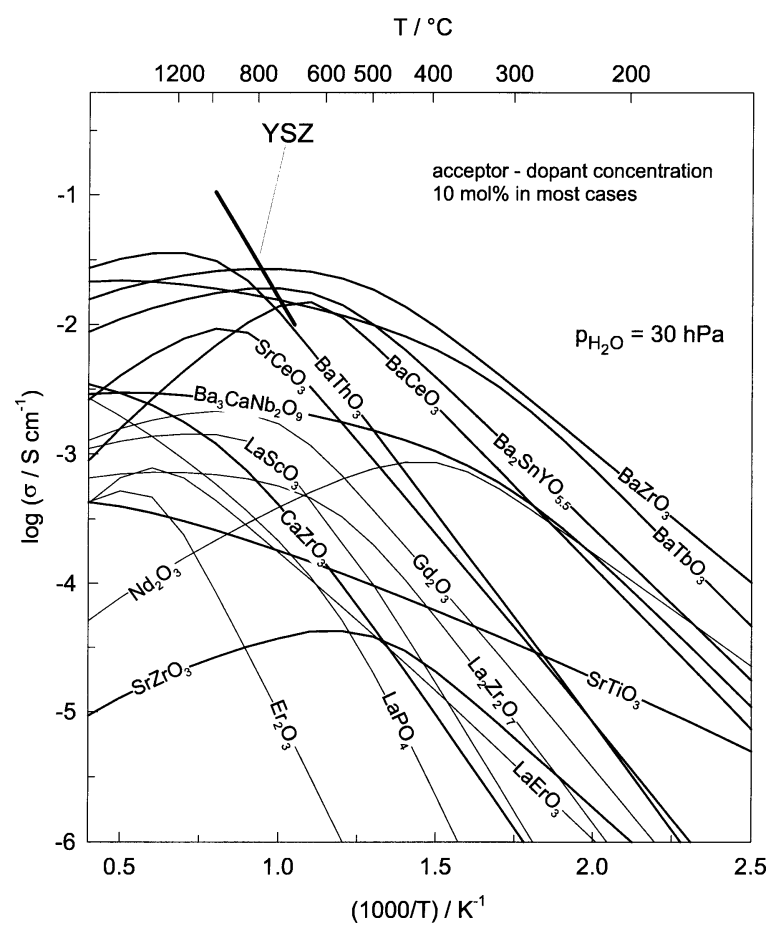

Figure 25. Proton conductivity of various oxides, as calculated from data on proton concentrations and mobilities, according to Norby and Larring (the type of dopant is not indicated; see ref 187 for source data). ${ }^{186}$ The conductivity of oxides with a perovskite-type structure are shown by bold lines, and the conductivity of the oxide ion conductor YSZ (yttria-stabilized zirconia) is shown for comparison. (reproduced with the kind permission of Annual Reviews, http://www.AnnualReviews.org)

the degree of sulfonation (expressed as equivalent weight or ion exchange capacity), and this is usually in the range of $800-1200 \mathrm{~g} /$ equiv $(0.83-1.25$ mequiv/ g) for perfluorinated polymers and 600-800 g/equiv (1.25-1.66 mequiv/g) for hydrocarbon separator materials. Despite the diversity of backbones and polymeric architectures, the transport properties are very similar, i.e., the transport coefficients, as a function of the water volume fraction, are similar to those of Nafion (as a representative of perfluorinated membrane materials) and sulfonated poly(arylene ether ketone)s (as a representative for hydrocarbon based membranes) (see Figures 19 and 20). Even the hydration isotherms (absorbed water per sulfonic acid group, as a function of RH) seem to be quite uniform. Only the water uptake in liquid water (or water/ methanol mixtures) may differ significantly. Particularly at elevated temperatures, there may be tremendous swelling, and the onset of exaggerated (partially irreversible) swelling is closely related to the mechanical properties of the polymers.

The transport properties that are most significantly affected by changes of the water volume fraction are the water/methanol el ectro-osmotic drag and permeation, both of which have significant contributions from viscous flow (see Section 3.2.1.1). For DMFC applications (where the membrane is in contact with a liquid water/methanol mixture), this type of transport determines the crossover, which is only acceptably low for solvent volume fractions smaller than $\sim 20$ vol \% (see Figures 14 and 15). Consequently, recent attempts have been focused on strengthening the polymer, e.g., by cross-linking ${ }^{307-315,321}$ or forming co-polymers of sulfonated arylenes and polyvinylidene difluoride (PVDF). ${ }^{322}$ I nitial fuel-cell tests are actually quite promising; ${ }^{313}$ however, the brittleness of highly cross-linked polymers may cause mechanical failure of the membrane. The formation of composites with isolated inorganic particles usually does not have a positive effect on the swelling properties. In the case of Nafion, it may suppress its crystallinity, which leads to an increased swelling. Nevertheless, improved performance under fuel-cell conditions has been reported in a few cases, 243,244 and better understanding of the observed effects may guide a more systematic improvement of composites. The design of microstructures with a hydrated sulfonated polymer confined in the pores of a stable well-connected (inorganic) matrix may be another promising approach. The mechanical requirements of such a matrix are actually quite severe, because of the need to compensate for the high pressure due to osmosis and electro-osmotic drag. Some specific interaction between the sulfonated polymer and the confining phase is required to prevent the first from dissolving. Currently, there are several attempts to build such microstructures, some of which are based on controlled precipitation from homogeneous solutions, ${ }^{323}$ and others that rely on sequential formation of the different parts of the microstructure, e.g., by precipitation of inorganic proton conductors in porous PTFE. ${ }^{245}$ Another degree of freedom that may be exploited is the degree of sulfonation across the membrane. In a DMFC, the anode side of the membrane, which is in contact with the methanol solution, may be prevented from swelling by locally reducing the degree of sulfonation (e.g., by laminating layers of different degree of sulfonation ${ }^{323}$ or by surface modification ${ }^{312}$ ), whereas an increased degree of sulfonation may prevent drying out on the anode side at high temperatures and low humidity conditions.

As explained in the Introduction, the latter conditions are of paramount importance for PEM fuel-cell technology; however, to date, there are no protonconducting sulfonated polymer membranes available that satisfy all requirements under these conditions (i.e., $\mathrm{T}>120{ }^{\circ} \mathrm{C}, \mathrm{RH}<25 \%$ ). The most obvious limitation is the low proton conductivity, as a result of low hydration levels under these conditions. Because of their superacidity, which results in high hydrophilicity (see Section 3.1.2.1), the highest equilibrium water contents are observed in perfluorosulfonic acid polymers. However, even for these, a water content of $2.5 \mathrm{H}_{2} \mathrm{O} / \mathrm{SO}_{3} \mathrm{H}$ (at $\mathrm{T}=144{ }^{\circ} \mathrm{C}, \mathrm{RH}=25 \%$; see Figure 26, bottom) is too low to effectively bridge the separation between neighboring sulfonate groups, which is $\sim 0.8 \mathrm{~nm}$ in Nafion (1100 g/equiv). ${ }^{212}$ Because of the poor connectivity (percolation) of the possible water structures and the strong local retardation of the water diffusion (see discussion in Section 3.2.1.1), fast proton conduction according to a vehicle mechanism is not possible at such low degrees of hydration. On the other hand, there is theor etical ${ }^{38,39}$ and experimental indication ${ }^{324}$ that high proton conductivity may be possible at low hydration levels, pro- 


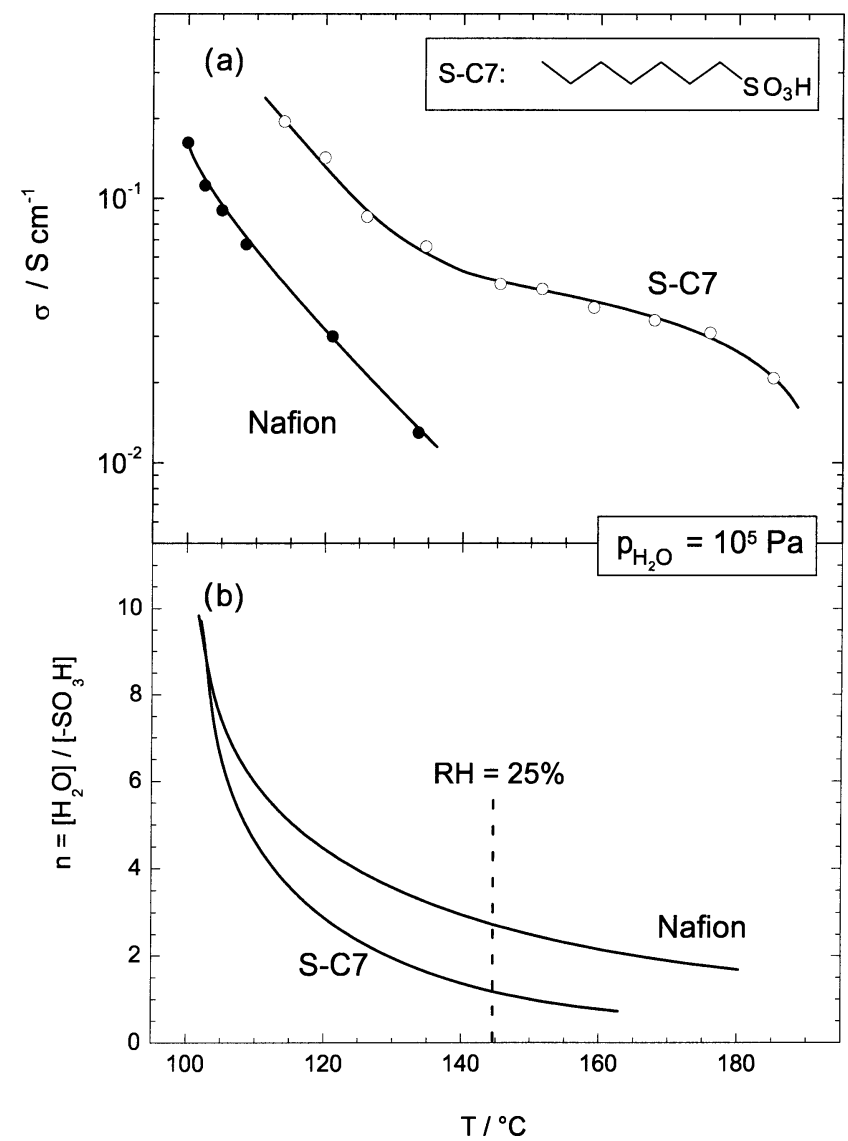

Figure 26. (a) Conductivity and (b) water uptake of $\mathrm{S}-\mathrm{C} 7$ (heptane terminated by a sulfonic acid functional group) and Nafion 117 ( $E W=1100$ g/equiv), as a function of temperature at a water partial pressure of $\mathrm{p}_{\mathrm{H}_{2} \mathrm{O}}=10^{5} \mathrm{~Pa}{ }^{324}$

vided that the sulfonic acid functional groups are less separated or at least more mobile. The top portion of Figure 26 shows the ionic conductivity of a sulfonated oligomer (heptyl sulfonic acid), which starts to show a decrease in conductivity at a higher temperature than that observed in Nafion, leveling off to a very high conductivity of $\sim 4 \times 10^{-2} \mathrm{~S} / \mathrm{cm}$ in the temperature range of $130-170{ }^{\circ} \mathrm{C}\left(\mathrm{p}_{\mathrm{H}_{2} \mathrm{O}}=10^{5}\right.$ $\mathrm{Pa}), 324$ which is reminescent of the conductivity behavior of aqueous highly concentrated sulfuric acid. ${ }^{325}$ The latter observation indicates that another proton conduction mechanism appears at low humidification levels. Recent electronic structure calculations of a two-side-chain fragment of the Dow membrane demonstrates that a hydration of three water molecules per sulfonic acid leads to dissociation and a configuration in which one of the excess protons is shared between two water molecules (the formation of a Zundel ion) and the other is a hydronium ion between the two sulfonate groups. ${ }^{23}$ For somewhat lower water contents, the affinities of the sulfonic acid group and the remaining water structure towards the proton tend to balance. The AIMD (see Section 2.2.3) of triflic acid monohydrate 38,39 indeed finds excess protons within the water structure, namely, as Zundel and hydronium ions, and shared between neighboring sulfonate groups (Figure 27). Such configurations may have a role in the mechanisms of proton conduction involving the dynamics of both the sulfonate groups and the water

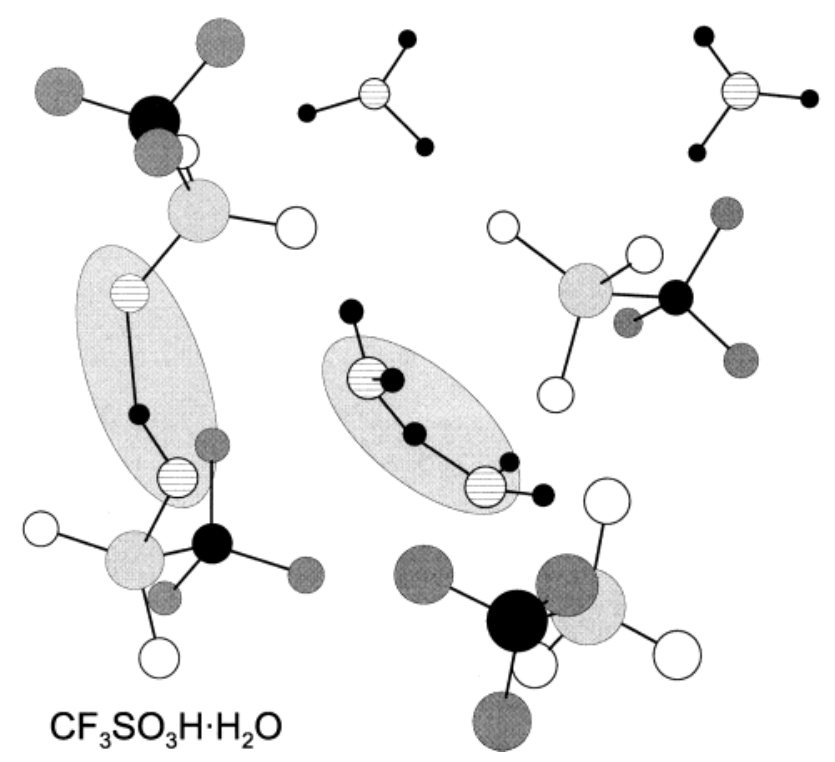

Figure 27. Defective structure of solid trifluoromethanesulfonic acid hydrate $\left(\mathrm{CF}_{3} \mathrm{SO}_{3} \mathrm{H} \cdot \mathrm{H}_{2} \mathrm{O}\right)_{4}$ found using ab initio molecular dynamics (AIMD; see Section 2.2.3 for a description of the technique), showing two hydronium ions hydrogen-bonded to sulfonate groups (as found in the perfect structure) but, more importantly, two "shared protons" (one between two sulfonate groups and the other as part of a Zundel ion; see text). Note that the energy of the defective structure is only $\sim 30 \mathrm{~kJ} / \mathrm{mol}$ higher than that of the perfect structure. ${ }^{23,38,39}$

molecules. Whether this is the case for the high conductivities of lowly hydrated sulfonic acid oligomers (Figure 26) and triflic acid hydrates is not yet clear (the high conductivities may also be the consequence of high self-diffusion coefficients of such ionic, highly fluid systems); however, these observations may justify an inclusion of highly sulfonated systems with significantly smaller $-\mathrm{SO}_{3} \mathrm{H}$ separations into future work. Polymers with more than one sulfonic acid group per phenyl ring are indeed possible (e.g., for poly(arylene sulfide)s) and interesting conductivities at low humidification have been reported. ${ }^{326}$ Of course, the formation of applicable separator materials requires the immobilization of such highly soluble polymers, e.g., in a manner previously described or by making them a constituent of a macromolecular structure with highly sulfonated and unsulfonated components in controlled morphol ogies (for a current review, see ref 327), which is a strategy pushed forward by several French groups,328-331 M cGrath et al., 332 and Miyatake et al. ${ }^{333}$ The latter authors actually reported the highest high-temperature conductivity for a sulfonated polyimide co-polymer that contained fluorenyl moieties, indicating a complex relation between chemistry, microstructure (packing), and proton conductivity for this class of polymers. Note that the typical block separation length for such polymers is on the order of 10-100 nm, i.e., it is well above the scale relevant for the transport mechanisms $(\sim 1 \mathrm{~nm})$, as discussed in Sections 3.1.2.1 and 3.2.1.

Unfortunately, the thermal stability of sulfonated systems is quite limited, particularly when the stabilizing effect of the hydration water is reduced (Section 3.1.2.1). The transport of water not only 
$\mathrm{T} /{ }^{\circ} \mathrm{C}$

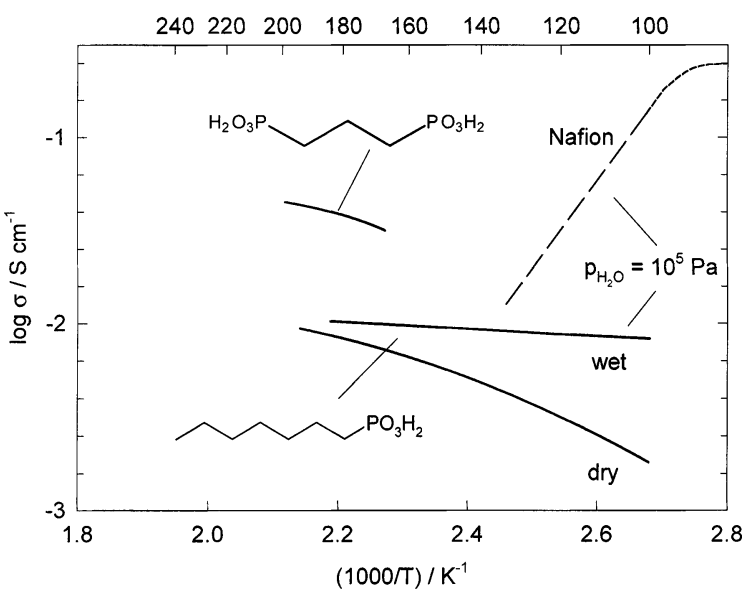

Figure 28. Conductivity of two phosphonic acid-terminated oligomers under dry and wet conditions, compared to the proton conductivity of Nafion at a water partial pressure of $\mathrm{p}_{\mathrm{H}_{2} \mathrm{O}}=10^{5} \mathrm{~Pa} .{ }^{324}$

requires water management but also leads to thermodynamic efficiency losses in fuel cells using such membranes as separators. A recent comparative study of oligomers terminated by different protogenic groups (sulfonic acid, imidazole, and phosphonic acid) shows that the more-amphoteric groups show a higher thermal and electrochemical stability in the dry state, ${ }^{324}$ where they still show significant proton conductivity. However, as discussed in Section 3.1.2.3, this conductivity seems to be limited to $\sim 10^{-2} \mathrm{~S} / \mathrm{cm}$ for systems based on heterocycles. The other problem with such systems is the high overpotentials for oxygen reduction at platinum cathodes, which already has been observed by Yang et al. ${ }^{234}$ For phosphonic acid functionalized systems, however, the conductivities in the dry state are $\sim 1$ order of magnitude higher, and, as opposed to most heterocycle-based systems, phosphonates have some remaining hydrophilicity. This allows for high proton conductivity at low temperatures (Figure 28) and may help to reduce cathodic overpotentials that are caused by the adsorption of phosphonic groups onto the platinum surface. Of course, this is also an inherent problem of systems that contain free $\mathrm{H}_{3} \mathrm{PO}_{4}$, such as the adducts of PBI and phosphoric acid (see Section 3.1.2.2).

Apart from the immobilization of the protogenic group as part of a polymeric architecture (e.g., soft side-chain polymer), ionic immobilization may be another interesting strategy to suppress the volatility of nonaqueous proton solvents. Some molten salts combine low vapor pressure with very high ionic conductivity. However, the latter is usually the consequence of a very high fluidity, resulting in high mobility of all ionic species in the systems. Watanabe et al. ${ }^{334}$ have actually reported a few molten salts that contain imidazolium or imidazol ium derivatives with very high ionic conductivity. However, the charge carriers are complex ions, which are very mobile as a whole, i.e., the conductivity is not protonic. Nevertheless, when combined with the spacer concept, ionic immobilization may al so become rel evant for the immobilization of (neutral) proton solvents. Spacer molecules may be terminated by a proton solvent (e.g., imidazole, phosphonic acid) at one end and by an ionic group at the other end, with the counter charge being just a single ion (this may lead to a liquid (molten) systems with low vapor pressure) or a poly-ion (which may lead to a plastic material).

The use of polymeric constituents becomes progressively critical with increasing temperature; therefore, for durability reasons, partially and even fully inorganic systems have recently attracted interest as proton-conducting separator materials.

Heteropolyacids are frequently used to modify proton-conducting composites, 252 or they are just dispersed in inert matrixes. ${ }^{335,336}$ However, because the proton conduction mechanism of such hydrated salts is similar to those of hydrated polymeric systems, ${ }^{337}$ these composites show qualitatively similar transport properties. The same is true for organically modified inorganic layered compounds such as titanium phosphate sulfophenylenphosphonate, the conductivity of which is dependent on the RH value, in a manner similar to that observed with Nafion. ${ }^{338}$

In contrast to this class of materials, acidic salts of oxo-acids may show a proton conduction mechanism, which is more related to that of liquid orthophosphoric acid (see Section 3.1.1.2). The prototypical compound is $\mathrm{CsHSO}_{4}$, which shows very high proton conductivity above a first-order phase transition at $\mathrm{T} \approx 140{ }^{\circ} \mathrm{C} .{ }^{339}$ The conducting phase is still a solid; that is, there is still long-range order but locally, there is a tremendous dynamic reorientational disorder of the sulfate tetrahedral and extended vibrations of both cesium and sulfate characteristic for socalled plastic phases. The hydrogen bonding between the sulfate tetrahedral is highly dynamic in the plastic phase, and almost completely self-dissociated: high proton mobility leading to high proton conductivity. ${ }^{1,10,144}$ Haile et al. tested such type of materials in $\mathrm{H}_{2} / \mathrm{O}_{2}$ fuel cells ${ }^{340}$ and recently also in methanol $/ \mathrm{O}_{2}$ fuel cells. ${ }^{341}$ The current densities obtained were limited by the protonic resistance of the el ectrolytes $\left(\mathrm{CsHSO}_{4}\right.$ and $\left.\mathrm{CsH}_{2} \mathrm{PO}_{4}\right)$, i.e., no cathodic overpotentials are reported, which is a true advantage, compared to other systems operating under similar conditions. The softness, high solubility in water, sensitivity to reduction, and the very low room-temperature conductivity are still severe disadvantages in these particular compounds; however, there is a plethora of solid acids, some of which may be suitable for fuel-cell applications.

Further increase in the operating temperature of a fuel cell is possible, using proton-conducting oxides as separator materials. Although initial fuel-cell tests (typically in the temperature range of $600-800{ }^{\circ} \mathrm{C}$ using pure gases) were quite promising, ${ }^{180-184}$ the combination of high bulk proton conductivity and stability has only recently been achieved.186,188,205 Yttrium-doped $\mathrm{BaZrO}_{3}$-based oxides actually show the highest bulk conductivity (Figure 25), and they have a high thermodynamic stability, with respect to reactions with acidic gases such as $\mathrm{CO}_{2} .{ }^{188}$ Problems are related to high grain-boundary impedances (which is probably the result of symmetry reduction in the grain-boundary region), current constriction 
$\mathrm{T} /{ }^{\circ} \mathrm{C}$

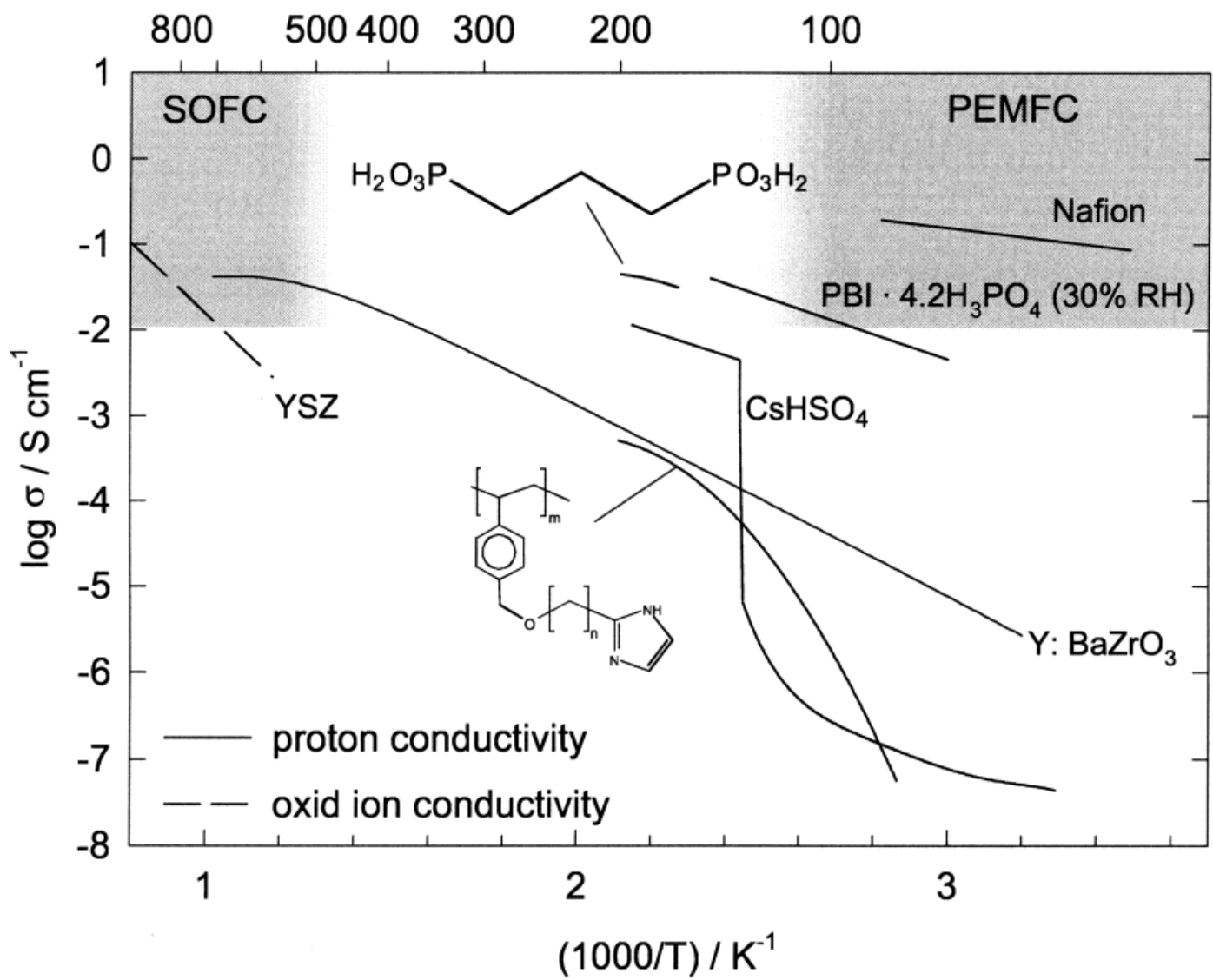

Figure 29. Conductivity of some intermediate-temperature proton conductors, compared to the conductivity of Nafion and the oxide ion conductivity of YSZ (yttria-stabilized zirconia), the standard electrolyte materials for low- and hightemperature fuel cells, proton exchange membrane fuel cells (PEMFCs), and solid oxide fuel cells (SOFCs).

effects, and high cathodic overpotentials at platinum el ectrodes. ${ }^{186}$ M ore-suitable electrocatalysts have not been devel oped thus far; ther efore, there is probably a huge potential for further improvements. An interesting feature of proton-conducting oxides is, that they may show both proton conductivity and chemical water diffusion under medium dry conditions (Section 3.2.2). This allows the transport of water electrochemically formed at the cathode, to migrate to the anode side, where it may be consumed in fuelreforming reactions. This approach has been suggested and confirmed by Coors ${ }^{342}$ and may become the basis for fuel cells operating with dry methane as a fuel.

The key to the development of $\mathrm{CO}_{2}$-resistant protonconducting oxides was the maximization of the entropic stabilization of protonic defects. ${ }^{188}$ If this approach also led to stable hydroxides with sufficiently high conductivity, AFCs using such el ectrolytes may operate even with air as the cathode gas. This would be tremendously advantageous, because fuel cells with nonacidic electrolytes may operate with non-noble-metal catalysts such as nickel for the anode and silver for the cathode.

The above-described qual itative considerations hopefully give a flavor for the complexity of the development of novel proton-conducting separator materials for fuel-cell applications. When solely considering proton conductivity, significant progress has been made over the past few years, and quite a few old and new materials have been reported that show interesting proton conductivities, especially in the intermediate temperature range (see Figure 29). ${ }^{343}$ Also, significant progress in the understanding of transport properties has also been made, which is, to a large extent, due to emerging simulation techniques (Section 2) as relatively recent research tools. The level of understanding reached so far has already helped to better control the transport properties of available proton-conducting materials, and surely will be of great help in a more directed search for novel proton-conducting materials for fuel-cell applications. This is indeed necessary, because, presently, Nafion materials, or modified derivatives of its structure with modified pendant chains, lower equivalent weights, or modified processing are still the benchmark PEMs, despite more than twenty years of research into alternatives.

\section{Acknowledgement}

The authors thank R. Merkle, E. Kotomin, and J . Fleig (all from the Max-Planck-Institut für Festkörperforschung) and the external reviewers for car efully reading the proofs and fruitful discussions. We thank A. Fuchs for assisting in making the figures and the Deutsche Forschungsgemeinschaft (KR 794), the Bundesministerium für Bildung und 
Forschung (0329567), and the E nergiestiftung BadenWürttemberg (A 19603) for financial support.

\section{References}

(1) Kreuer, K. D. Chem. Mater. 1996, 8, 610

(2) Colomban, P. Ann. Chim.-Sci. Mater. 1999, 24, 1

(3) Vielstich, W., Lamm, A., Gasteiger, H. A., Eds. Handbook of Fuel Cells-F undamentals, Technol ogy and Applications; Wiley: Chichester, U.K., 2003.

(4) Annu. Rev. Mater. Res. 2003, 33. (Special issue, Materials for Fuel Cells.)

(5) Savadogo, O. J . New Mater. Electrochem. Syst. 1998, 1, 47.

(6) Li, Q.; He, R.; J ensen, J . O.; Bjerrum, N. J . Chem. Mater. 2003, 15,4896

(7) Rikukawa, M.; Sanui, K. Prog. Polym. Sci. 2000, 25, 1463.

(8) Rozière, J .; J ones, D. J . Annu. Rev. Mater. Res. 2003, 33, 503.

(9) J annasch, P. Curr. Opin. Coll loid Interface Sci. 2003, 8, 96.

(10) Münch, W.; Kreuer, K. D.; Traub, U.; Maier, J . J . Mol. Struct. (THEOCHEM) 1996, 381, 1.

(11) Büchi, F. N.; Huslage, J ., Scherer, G. G. PSI Annu. Rep. 1997 Annex V, 48

(12) Springer, T. E.; Zawodzinski, T. A.; Gottesfeld, S. J . Electrochem. Soc. 1991, 138, 2334

(13) Fuller, T. F.; Newman, J . J . Electrochem. Soc. 1991, 140, 1218.

(14) Eikerling, M.; Kharkats, Y. I.; Kornyshev, A. A.; Volfkovich, Y M. J. Electrochem. Soc. 1998, 145, 2677.

(15) Paddison, S. J .; Pratt, L. R.; Zawodzinski, T.; Reagor, D. W. Fluid Phase Equilib. 1998, 150-151, 235

(16) Paddison, S. J .; Pratt, L. R.; Zawodzinski, T. A., J r. In Proton Conducting Membrane Fue Cells II; Gottesfeld, S., Fuller, T. F., Eds.; The Electrochemical Society Proceedings Series 98-27; The Electrochemical Society: Pennington, NJ , 1999; p 99.

(17) Paddison, S. J .; Zawodzinski, T. A., J r. Solid State I onics 1998 $113-115,333$

(18) Paddison, S. J .; Pratt, L. R.; Zawodzinski, T. A., J r. J . New Mater Electrochem. Syst. 1999, 2, 183.

(19) Paddison, S. J .; Pratt, L. R.; Zawodzinski, T. A., J r. J . Phys. Chem. A 2001, 105, 6266.

(20) ) Paddison, S. J . J . New Mater. Electrochem. Syst. 2001, 4, 197

(21) Eikerling, M.; Paddison, S. J .; Zawodzinski, T. A., J r. J . New Mater. Electrochem. Syst. 2002, 5, 15

(22) Paddison, S. J. In Handbook of Fuel Cells Fundamentals Technology and Applications: Volume 3, Fuel Cell Technology and Applications: Part 1; Vielstich, W., Lamm, A., Gasteiger H. A., Eds.; Wiley: Chichester, U.K., 2003; p 396.

(23) Paddison, S. J. Annu. Rev. Mater. Res. 2003, 33, 289.

(24) J ohansson, P.; Tegenfeldt, J .; Lindgren, J . Electrochim. Acta 2000, 45, 3055

(25) Li, T.; Wlaschin, A.; Bal buena, P. B. Ind. Eng. Chem. Res. 2001 40, 4789.

(26) Spohr, E.; Commer, P.; Kornyshev, A. A. J . Phys. Chem. B 2002, $106,10560$.

(27) Commer, P.; Cherstvy, A. G.; Spohr, E.; Kornyshev, A. A. Fue Cells 2002, 2, 127.

(28) Spohr, E. Mol. Simul. 2004, 30, 107.

(29) Commer, P.; Hartnig, C.; Seeliger, D.; Spohr, E., submitted to Mol. Simul.

(30) Warshel, A. Computer Modeling of Chemical Reactions in Enzymes and Solutions; Wiley: New York, 1991.

(31) Warshel, A. J . Phys. Chem. 1982, 86, 2218.

(32) Warshel, A.; Weiss, R. M. J . Am. Chem. Soc. 1980, 102, 6218

(33) Tuckerman, M.; Laasonen, K.; Sprik, M.; Parrinello, M. J . Phys. Chem. 1995, 99, 5749.

(34) Tuckerman, M.; Laasonen, K.; Sprik, M.; Parrinello, M. J . Chem. Phys. 1995, 103, 150.

(35) Tuckerman, M. E.; Marx, D.; Klein, M. L.; Parrinello, M. Science 1997, 275, 817.

(36) Marx, D.; Tuckerman, M. E.; Hutter, J .; Parrinello, M. Nature 1999, 397, 601.

(37) Münch, W.; Kreuer, K.-D.; Silvestri, W.; Maier, J .; Seifert, G. Solid State I onics 2001, 145, 437.

(38) Eikerling, M.; Paddison, S. J .; Pratt, L. R.; Zawodzinski, T. A., J r. Chem. Phys. Lett. 2003, 368, 108.

(39) Paddison, S. J .; Pratt, L. R., submitted to Mol. Phys., 2004.

(40) Paddison, S. J.: Paul, R.; Zawodzinski, T. A., J r. In Proton Conducting Membrane Fue Cells II; Gottesfeld, S., Fuller, T. F., Eds.; The Electrochemical Society Proceedings Series 98-27; The Electrochemical Society: Pennington, NJ , 1999; p 106.

(41) Paddison, S. J .; Paul, R.; Zawodzinski, T. A., J r. J . Electrochem. Soc. 2000, 147, 617

(42) Paddison, S.J .; Paul, R.; Zawodzinski, T. A., J r. J . Chem. Phys 2001, 115, 7753.

(43) Paddison, S. J .; Paul, R.; Pivovar, B. S. In Direct Methanol Fuel Cells; Narayanan, S., Gottesfeld, S., Zawodzinski, T. A., Eds. The Electrochemical Society Proceedings Series 01-04; The Electrochemical Society: Pennington, NJ , 2001; p 8.
(44) Paddison, S. J .; Paul, R.; Kreuer, K. D.; Zawodzinski, T. A., J r In Direct Methanol Fuel Cells; Narayanan, S., Gottesfeld, S. Zawodzinski, T. A., Eds.; The Electrochemical Society Proceedings Series 01-04: The Electrochemical Society: Pennington, NJ , 2001; p 29.

(45) Paddison, S.J .; Paul, R.; Kreuer, K. D. Phys. Chem. Chem. Phys. 2002, 4, 1151.

(46) Paddison, S. J .; Paul, R. Phys. Chem. Chem. Phys. 2002, 4, 1158.

(47) Paul, R.; Paddison, S. J . In Advances in Materials Theory and Modeling-Bridging Over MultipleLength and Time Scales; Bulatov, V., Colombo, L., Cleri, F., Lewis, L. J ., Mousseau, N., Eds.; Materials Research Society: Warrendale, PA, 2001; pp AA7.16.1.

48) Paul, R.; Paddison, S. J J . Chem. Phys. 2001, 115, 7762

(49) Paul, R.; Paddison, S. J. Phys. Rev. E 2003, 67, 016108-1.

(50) Paul, R.; Paddison, S. J. Solid State I onics 2004, 168, 245

51) Paul, R.; Paddison, S. 」. J . Phys. Chem. B 2004, 108, 1323113241.

(52) Szabo, A.; Ostlund, N. S. Modern Quantum Chemistry: Introduction to Advanced Electronic Structure Theory; McGrawHill: New York, 1989.

(53) McWeeny, R. Methods of Molecular Quantum Mechanics; Academic Press: London, 1989.

54) Hehre, W. I .; Radom, L.; Schleyer, P. V. R.; Pople, J. A. Ab Initio Molecular Orbital Theory; Wiley: New York, 1986.

(55) Born, M.; Oppenheimer, J. R. Ann. Phys. 1927, 79, 361.

(56) Hartree, D. R. Proc. Cambridge Philos. Soc. 1928, 24, 328.

(57) Fock, V. A. Z. Phys. 1930, 15, 126.

(58) Møller, C.; Plesset, M. S. Phys. Rev. 1934, 46, 618.

59) Shavitt, I. In Modern Theoretical Chemistry; Schaefer, H. F., Ed. Plenum Press: New York, 1977.

(60) Boys, S. F. Proc. R. Soc. London A 1950, A200, 542.

(61) Parr, R. G.; Yang, W. Density Functional Theory of Atoms and Molecules; Oxford: New York, 1989.

(62) Hohenberg, P.; Kohn, W. Phys. Rev. B 1964, 136, B864.

(63) Kohn, W.; Sham, L. J. Phys. Rev. 1965, 140, A1133.

(64) Lee, C.; Yang, W.; Parr, R. G. Phys. Rev. B 1988, 37, 785.

(65) Perdew, J. P. Phys. Rev. B 1986, 33, 8822; 1986, 34, 7406.

(66) Perdew, J. P. In Electronic Structure of Solids; Ziesche, P. Eschrig, H., Eds.; Academie Verlag: Berlin, 1991

(67) Becke, A. D. Phys. Rev. 1988, 38, A3098.

(68) J ohnson, B. G.; Gill, P. M. W.; Pople, J . A. J . Chem. Phys. 1993, 98, 5612.

(69) Porezag, D.; Frauenheim, T.; Köhler, T.; Seifert, G.; Kaschner R. Phys. Rev. B 1995, 51, 12947.

(70) Seifert, G.; Porezag, D.; Frauenheim, T. Int. J . Quantum Chem. 1996, 58, 185

(71) Frauenheim, T.; Seifert, G.; Elstner. M.; Niehaus, T. A.; Köhler T.; Amkreutz, M.; Sternberg, M.; Hajnal, Z.; di Carlo, A.; Suhai, S. J . Phys. C 2002, 14, 3015.

(72) Seifert, G. In Encycl opedia of Computational Chemistry; Wiley: New York, in press.

(73) Münch, W.; Kreuer, K. D.; Seifert, G.; Maier, J . Solid Statel onics 2000, 136-137, 183

(74) Allen, M. P.; Tildesley, D. J . Computer Simulation of Liquids; Clarendon Press: Oxford, U.K., 1987.

(75) Gray, C. G.; Gubbins, K. E. Theory of Molecular Fluids; Clarendon Press: Oxford, U.K., 1984; Vol. 1.

(76) Remler, D. K.; Madden, P. A. Mol. Phys. 1990, 70, 921

(77) Payne, M. C.; Teter, M. P.; Allan, D. C.; Arias, T. A.; J oannopouIos, J. D. Rev. Mod. Phys. 1992, 64, 1045.

(78) Metropolis, N. A.; Rosenbluth, A. W.; Rosenbluth, M. N.; Teller A. H.; Teller, E. J . Chem. Phys. 1953, 21, 1087.

(79) Alder, B. J Wainwright, T. E. J . Chem. Phys. 1957, 27, 1208

(80) Dick, B. G.; Overhauser, A. W. Phys. Rev. 1958, 112, 90.

(81) Halley, J. W.; Rustad, J. R.; Rahman, A. J . Chem. Phys. 1993 $98,4110$.

(82) Hammes-Schiffer, S.; Tully, J . C. J . Chem. Phys. 1994, 101, 4657.

(83) Vuilleumier, R.; Borgis, D. J . Mol. Struct. (THEOCHEM) 1997 436, 555

(84) Vuilleumier, R.; Borgis, D. J . Phys. Chem. B 1998, 102, 4261.

(85) Vuilleumier, R.; Borgis, D. Chem. Phys. Lett. 1998, 284, 71.

(86) Vuilleumier, R.; Borgis, D. Isr. J . Chem. 1999, 39, 457.

87) Vuilleumier, R.; Borgis, D. J . Chem. Phys. 1999, 111, 4251.

(88) Schmitt, U. W.; Voth, G. A. J . Phys. Chem. B 1998, 102, 5547.

(89) Schmitt, U. W.; Voth, G. A. J . Chem. Phys. 1999, 111, 9361.

(90) Schmitt, U. W.; Voth, G. A. I sr. J . Chem. 1999, 39, 483.

(91) Schmitt, U. W.; Voth, G. A. Chem. Phys. Lett. 2000, 329, 36

(92) Kim, J.; Schmitt, U. W.; Gruetzmacher, J. A.; Voth, G. A.; Scherer, N. F. J . Chem. Phys. 2000, 116, 737.

(93) Day, T. J . F.; Soudackov, A. V.; Cuma, M.; Schmitt, U. W.; Voth, G. A. J . Chem. Phys. 2002, 117, 5839.

(94) Kornyshev, A. A.; Wal bran, S. J . Chem. Phys. 2001, 114, 10039.

(95) Kresse, G.; Hafner, J. Phys. Rev. B 1993, 47, 558.

(96) Kresse, G.; Hafner, J. Phys. Rev. B 1994, 49, 14251.

(97) Kresse, G.; Furthmüller, J . Phys. Rev. B 1996, 54, 11169.

(98) Car, R.; Parrinello, M. Phys. Rev. Lett. 1985, 55, 2471.

(99) Eikerling, M.; Kornyshev, A. A. J . Electroanal. Chem. 2001, 502, 1. 
(100) Eikerling, M.; Kornyshev, A. A.; Kuznetsov, A. M.; Ulstrup, J .; Walbran, S. J. Phys. Chem. B 2001, 105, 3646.

(101) Harned, H. S.; Owen, B. B. ThePhysical Chemistry of Electrolyte Solutions; Reinhold: New York, 1958.

(102) Wolynes, P. G. Annu. Rev. Phys. Chem. 1980, 31, 345.

(103) Kay, R. L. In Water, A ComprehensiveTreatise; Franks, F., Ed.; Plenum Press: New York, 1973; Vol. 3.

(104) Robinson, R. A.; Stokes, R. H. ElectrolyteSol utions, 2nd Edition; Butterworth: London, 1959

(105) Born, M. Z. Phys. 1920, 1, 221.

(106) Fuoss, R. M. Proc. Natl. Acad. Sci. 1959, 45, 807.

(107) Boyd, R. H. J . Chem. Phys. 1961, 35, 1281

(108) Zwanzig, R. J . Chem. Phys. 1963, 38, 1603; 1970, 52, 3625

(109) Hubbard, J . B.; Onsager, L. J . Chem. Phys. 1977, 67, 4850.

(110) Hubbard, J. B. J . Chem. Phys. 1978, 68, 1649

(111) Wolynes, P. G. J. Chem. Phys. 1978, 68, 473.

(112) Colonomos, P.; Wolynes, P. G. J . Chem. Phys. 1979, 71, 2644.

(113) Chen, J. H.; Adelman, S. A. J . Chem. Phys. 1980, 72, 2819.

(114) Chong, S.-H.; Hirata, F. J . Chem. Phys. 1998, 108, 7339.

(115) Biswas, B.; Roy, S.; Bagchi, B. Phys. Rev. Lett. 1995, 75, 1098

(116) Biswas, B.; Bagchi, B. J . Chem. Phys. 1997, 106, 5587; J . Am Chem. Soc. 1997, 119, 5946.

(117) Bagchi, B. J . Chem. Phys. 1998, 109, 3989.

(118) Resibois, P. M. V. ElectrolyteTheory: An Elementary Introduction to a Microscopic Approach; Harper and Row: New York, 1968

(119) Belletete, M.; Lachapelle, M.; Durocher, G. J . Phys. Chem. 1990, 94, 5337

(120) Guha Ray, J.; Sengupta, P. K. Chem. Phys. Lett. 1994, 230, 75.

(121) Das, K.; Sarkar, N.; Das, S.; Datta, A.; Bhattacharyya, K. Chem. Phys. Lett. 1996, 249, 323.

(122) Datta, A.; Mandal, D.; Pal, S. K.; Bhattacharyya, K. J . Phys. Chem. B 1997, 101, 10221.

(123) Faeder, J .; Ladanyi, B. M. J . Chem. Phys. 2000, 104, 1033

(124) Roux, B.; Karplus, M. Annu. Rev. Biophys. Biomol. Struct. 1994, $23,731$.

(125) Breed, J .; Sankararamakrishnan, R.; Kerr, I. D.; Sansom, M. S. P. Biophys. J. 1996, 70, 1643.

(126) Senapati, S.; Chandra, A. J . Phys. Chem. B 2001, 105, 5106.

(127) Booth, F. J . Chem. Phys. 1950, 19, 391

(128) Bontha, j. R.; Pintauro, P. N. Chem. Eng. Sci. 1994, 49, 3835.

(129) Steiner, T. Angew. Chem. 2002, 114, 50

(130) Dippel, T.; Hainovsky, N.; Kreuer, K. D.; Münch, W.; Maier, J . Ferroel ectrics 1995, 167, 59.

(131) Kreuer, K. D. Solid State I onics 2000, 136-137, 149

(132) Desiraju, G. R. Angew. Chem., Int. Ed. Engl. 1995, 34, 2311.

(133) Desiraju, G. R. Chem. Commun. 1997, (16), 1475.

(134) Schmidt, G. M. J . Pure Appl. Chem. 1971, 27, 647.

(135) Weissbuch, I.; Popovitz-Biro, R.; Lahav, M.; Leiserovitz, L. Acta Crystallogr. B: Struct. Sci. 1995, 51, 115.

(136) McDonald, j. C.; Whitesides, G. M. Chem. Rev. 1994, 94, 2383.

(137) Eigen, M.; De Maeyer, L. Proc. R. Soc. (London), Ser. A 1958, 247, 505 .

(138) Eigen, M. Angew. Chem. 1963, 75, 489

(139) Zundel, G.; Metzger, H. Z. Naturforsch. 1967, 22a, 1412.

(140) Agmon, N. Chem. Phys. Lett. 1995, 244, 456.

(141) Dippel, T.; Kreuer, K. D. Solid State Ionics 1991, 46, 3

(142) Cohen, B.; Huppert, D. J . Phys. Chem. A 2003, 107, 3598.

(143) Agmon, N. Isr. J. Chem. 1999, 39, 493.

(144) Kreuer, K. D. Solid State Ionics 1997, 94, 55.

(145) Sadeghi, R. R.; Cheng, H. P. J . Chem. Phys. 1999, 111, 2086.

(146) Cui, Q.; Karplus, M. J. Phys. Chem. B 2003, 107, 1071.

(147) Kreuer, K. D.; Rabenau, A.; Weppner, W. Angew. Chem., Int. Ed. Engl. 1982, 21, 208.

(148) Agmon, N. J . Chim. Phys. Phys.-Chim. Biol. 1996, 93, 1714.

(149) Tuckerman, M. E.; Marx, D.; Parrinello, M. Nature 2002, 417, 925.

(150) Trout, B. L.; Parrinello, M. J . Phys. Chem. B 1999, 103, 7340.

(151) Asthagiri, D.; Pratt, L. R.; Kress, J. D.; Gomez, M. A. Chem Phys. Lett. 2003, 380, 530.

(152) Asthagiri, D.; Pratt, L. R.; Kress, J. D.; Gomez, M. A. Proc. Natl. Acad. Sci. (USA) 2004, 101, 7233.

(153) Chen, B.; Park, J. M.; Ivanov, I.; Tabacchi, G.; Klein, M. L.; Parrinello, M. J. Am. Chem. Soc. 2002, 124, 8534

(154) Kuo, J . L.; Ciobanu, C. V.; Ojamäe, L.; Shavitt, I.; Singer, S. J . J. Chem. Phys. 2003, 118, 3583.

(155) Munson, R. A. J . Phys. Chem. 1964, 68, 3374

(156) Dippel, T.; Kreuer, K. D.; Lassègues, J . C.; Rodriguez, D. Solid State I onics 1993, 61, 41

(157) Spaeth, M.; Kreuer, K. D.; Dippel, T.; Maier, J . Solid Statel onics 1997, 97, 291.

(158) Spaeth, M.; Kreuer, K. D.; Maier, J . J . Solid State Chem. 1999 $148,169$.

(159) Chung, S. H.; Bajue, S.; Greenbaum, S. G. J . Chem. Phys. 2000 112,8515

(160) Schlechter, A.; Savinell, R. F. Solid State I onics 2002, 147, 181.

(161) Kawada, A.; McGhie, A. R.; Labes, M. M. J . Chem. Phys. 1970, 52,3121

(162) Decoursey, T. E. Phys. Rev. 2003, 83, 475.
(163) Zundel, G.; Weidemann, E. G. Eur. Biophys. Congr., Proc., 1st $1971,6,43$.

(164) Riehl, N. Trans. N.Y. Acad. Sci. 1965, 27, 772.

(165) Will, G. Z. Kristallogr. 1969, 129, 211.

(166) Brown, G. P.; Aftergut, S. J . Chem. Phys. 1963, 38, 1356.

(167) Pigon, K.; Chojnacki, H. Acta Phys. Pol. 1967, 31, 1069.

(168) McGhie, A. R.; Blum, H.; Labes, M. M. J . Chem. Phys. 1970, 52 6141

(169) Hickman, B. S.; Mascal, M.; Titman, J . J .; Wood, I. G. J . Am. Chem. Soc. 1999, 121, 11486

(170) Kreuer, K. D.; Fuchs, A.; I se, M.; Spaeth, M.; Maier, J . Electrochim. Acta 1998, 43, 1281.

(171) Kreuer, K. D. Solid State I onics: Science \& Technology; Chowdari, B. V. R., Lal, K., Agnihotry, S. A., Khare, N., Sekhon, S. S., Srivastava, P. C., Chandra, S., Eds.; World Scientific: Singapore, 1998, p 263

(172) Daycock, J. T.; J ones, G. P.; Evans, J. R. N.; Thomas, J. M. Nature 1968, 218, 673.

(173) Tatara, W.; Wojcik, M. J .; Lindgren, J .; Probst, M. J . Phys. Chem A 2003, 107, 7827

(174) Schuster, M.; Kreuer, K. D.; Maier, J ., in preparation.

(175) Toda, F.; Tanaka, K.; Foces-Foces, C.; Llamas-Saiz, A. L.; Limbach, H. H.; Aguilar-Parrilla, F.; Claramunt, R. M.; López, C.: Elguero, J. I . Chem. Soc. Chem. Commun. 1993, 1139.

(176) Stotz, S.; Wagner, C. Ber. Bunsen-Ges. Phys. Chem. 1966, 70, 781.

(177) Takahashi, T.; I wahara, H. 1980. Rev. Chim. Minér. 1980, t17, 243.

(178) I wahara, H.; Uchida, H.; Tanaka, S. Solid State Ionics 1983 $9-10,1021$.

(179) I wahara, H.; Uchida, H.; Ono, K.; Ogaki, K. J . Electrochem. Soc 1988, 135, 529

(180) I wahara, H. Solid State I onics 1988, 28-30, 573.

(181) I wahara, H.; Uchida, H.; Morimoto, K. J . Electrochem. Soc. 1990, 137,462

(182) Bonanos, N.; Ellis, B.; Mahmood, M. N. Solid Statel onics 1991 $44,305$.

(183) Tanigushi, N.; Hatoh, K.; Niikura, J .; Gamo, T. Solid Statel onics 1992, 53-56, 998.

(184) I wahara, H.; Yashima, T.; Hibino, T.; Ushida, H.J . Electrochem. Soc. 1993, 140, 1687

(185) Bonanos, N.; Knight, K. S.; Ellis, B. Solid Statel onics 1995, 79, 161.

(186) Kreuer, K. D. Annu. Rev. Mater. Res. 2003, 33, 333.

(187) Norby, T.; Larring, Y. Curr. Opin. Solid State Mater. Sci. 1997, 2, 593 .

(188) Kreuer, K. D. Solid State Ionics 1999, 125, 285

(189) Münch, W.; Seifert, G.; Kreuer, K. D.; Maier, J . Solid Statel onics 1996, 86-88, 647.

(190) Shimojo, F.; Hoshino, K.; Okazaki, H.; J . Phys. Soc. J pn. 1997 66,8

(191) Shimojo, F.; Hoshino, K. Solid State Ionics 2001, 145, 421.

(192) Pionke, M.; Mono, T.; Schweika, W.; Springer, T.; Schober, T. Solid State I onics 1997, 97, 497.

(193) Matzke, T.; Stimming, U.; Karmonik, C.; Soetramo, M.; Hempelmann, R.; Güthoff, F. Solid State I onics 1996, 86-88, 621.

(194) Hempelmann, R.; Soetramo, M.; Hartmann, O.; Wäppling, R. Solid State I onics 1998, 107, 269.

(195) Münch, W.; Seifert, G.; Kreuer, K. D.; Maier, J . Solid Statel onics 1997, 97, 39.

(196) Kreuer, K. D.; Münch, W.; Traub, U.; Maier, J . Ber. BunsenGes. Phys. Chem. 1998, 102, 552.

(197) Kreuer, K. D. Solid State I onics 1997, 97, 1.

(198) Sata, N.; Hiramoto, K.; I shigame, M.; Hosoya, S.; Niimura, N.; Shin, S. Phys. Rev. B 1996, 54, 15795.

(199) Münch, W.; Kreuer K. D.; Seifert, G.; Maier, J . Solid Statel onics 2000, 136-137, 183.

(200) Münch, W.; Kreuer K. D.; Seifert, G.; Maier, J . Solid Statel onics 1999, 125, 39

(201) Nowick, A. S. Solid State Ionics 1997, 97, 17.

(202) Kreuer, K. D.; Fuchs, A.; Maier, J . Solid State I onics 1995, 77 157.

(203) Münch, W.; Kreuer, K. D.; Adams, S.; Seifert, G.; Maier, J . Phase Transitions 1999, 68, 567.

(204) Ervine, J . T. S.; Corcoran, D. J . D.; Canales-Vasques, J . Solid State I onics 2002, 152, 749.

(205) Kreuer, K. D.; Adams, S.; Fuchs, A.; Klock, U.; Münch, W.; Maier Solid State I onics 2001, 145, 295.

(206) Kreuer, K. D.; Münch, W.; Ise, M.; He, T.; Fuchs, A.; Traub, U.; Maier, J Ber. Bunsen-Ges. Phys. Chem. 1997, 101, 1344.

(207) Nowick, A. S.; Yang, Du; Liang, K. C. Solid State Ionics 1999 $125,303$.

(208) Gebel, G.; Aldebert, P.; Pineri, M. Polymer 1993, 34, 333.

(209) Gebel, G.; Lambard, J. Macromol ecules 1997, 30, 7914.

(210) Gebel, G. Polymer 2000, 41, 5829.

(211) Rubalat, L.; Rollet, A. L.; Diat, O.; Gebel, G. J . Phys. IV 2002, 12 (PR6), 197.

(212) Ise, M. Ph.D. Thesis, University of Stuttgart, Stuttgart, Germany, 2000 
(213) Kreuer, K. D. J . Membr. Sci. 2001, 185, 29.

(214) Elliott, J. A.; Hanna, S.; Elliott, A. M. S.; Cooley, G. E. Macromol ecules 2000, 33, 4161.

(215) Khalatur, P. G.; Talitskikh, S. K.; Khokhlov, A. R. Macromol. Theory Simul. 2002, 11, 566.

(216) Edmondson, C. A.; Fontanella, J .J . Solid Statel onics 2002, 152153,355

(217) Haubold, H. G.; Vad, T.; J ungbluth, H.; Hiller, P. Electrochim Acta 2001, 46, 1559.

(218) Kazansky, V.; Solkan, V. Phys. Chem. Chem. Phys. 2003, 5, 31

(219) Kreuer, K. D. in Handbook of Fuel Cells-Fundamentals, Technol ogy and Applications; Vielstich, W., Lamm, A., Gasteiger, H. A. Eds.; Wiley: Chichester, U.K., 2003; p 420.

(220) Eikerling, M.; Kornyshev, A. A.; Stimming, U. J . Phys. Chem. B 1997, 101, 10807.

(221) Corry, B.; Kuyucak, S.; Chung, S. H. Chem. Phys. Lett. 2000 320, 35.

(222) Paddison, S. J .; Bender, G.; Kreuer, K. D.; Nicoloso, N.; Zawodzinski, T. A. J . New Mater. Electrochem. Syst. 2000, 3, 291.

(223) Paddison, S.J .; Reagor, D. W.; Zawodzinski, T. A. J . Electroanal. Chem. 1998, 459, 91

(224) Kreuer, K. D.; Dippel, T.; Meyer, W.; Maier, J . Mater. Res. Soc Symp. Proc. 1993, 293, 273.

(225) Rollet, A. L.; Simonin, J. P.; Turq, P.; Gebel, G.; Kahn, R. Vandais, A.; Noël, J . P.; Malveau, C.; Canet, D. J . Phys. Chem 2001, 105, 4503

(226) Zawodzinski, T. A.; Springer, T. E.; Davey, J .; J estel, R.; Lopez, C.; Valerio, J .; Gottesfeld, S. J . Electrochem. Soc. 1993, 140, 1981

(227) Kreuer, K. D.; Dippel, T.; Maier, J . Proc. Electrochem. Soc. 1995 95-23, 241.

(228) Cappadonia, M.; Erning, J. W.; Niaki, S. M. S.; Stimming, U. Solid State I onics 1995, 77, 65.

(229) Wainright, J . S.; Wang, J . T.; Weng, D.; Savinell, R. F.; Litt, M. J. Electrochem. Soc. 1995, 142, L121.

(230) Glipa, X.; Bonnet, B.; Mula, B.; J ones, D. J .; Rozière, J . J . Mater. Chem. 1999, 9, 3045.

(231) Bouchet, R.; Siebert, E. Solid State I onics 1999, 118, 287.

(232) Benicewicz, B. C. Presented as a poster during the 2003 Gordon Research Conference on Fuel Cells, Bristol, RI, J uly 27-August $1,2003$.

(233) Bozkurt, A.; Ise, M.; Kreuer, K. D.; Meyer, W. H.; Wegner, G. Solid State I onics 1999, 125, 225.

(234) Yang, C.; Costamagna, P.; Srinivasan, S.; Benziger, J .; Bocarsly, A. B. J. Power Sources $2001,103,1$

(235) Bozkurt, A.; Meyer, W. H.; Wegner, G. J . Power Sources 2003, 123,126

(236) Münch, W., unpublished data.

(237) Schuster, M. F.: Meyer, W. H.: Wegner, G.; Herz, H. G.; Ise, M.; Kreuer, K. D.; Maier, J. Solid State Ionics 2001, 145, 85.

(238) Schuster, M. F. H.; Meyer, W. H. Annu. Rev. Mater. Res. 2003 33, 233.

(239) Schuster, M. F. H.; Meyer, W. H.; Schuster, M.; Kreuer, K. D. Chem. Mater. 2004, 16, 329.

(240) Goward, G. R.; Schuster, M. F. H.; Sebastiani, D.; Schnell, I.; Spiess, H. W. J. Phys. Chem. B 2002, 106, 9322.

(241) Herz, H. G.; Kreuer, K. D.; Maier, J .; Scharfenberger, G. Schuster, M. F. H.; Meyer, W. H. Electrochim. Acta 2003, 48, 2165.

(242) Perrson, J. C. I annasch, P. Chem. Mater. 2003, 15, 3044.

(243) Miyake, N.; Wainright, J . S.; Savinell, R. F. J . Electrochem. Soc. A 2001, 148, A898.

(244) Adjemian, K. T.; Lee, S. J .; Srinvasan, S.; Benziger, J .; Bocarsly, A. B. J . Electrochem. Soc. A 2002, 149, A256.

(245) Alberti, G.; Casciola, M. Annu. Rev. Mater. Res. 2003, 33, 129.

(246) Kumar, B.; Fellner, J. P. J . Power Sources 2003, 123, 132.

(247) Mauritz, K. A.; Warren, R. M. Macromolecules 1989, 22, 1730.

(248) Costamagna, P.; Yang, C.; Bocarsly, A. B.; Srinivasan, S. Electrochim. Acta 2002, 47, 1023.

(249) Watanabe, M.; Uchida, H.; Seki, Y.; Emori, M. J . Electrochem Soc. 1996, 143, 3847

(250) Malhotra, S.; Datta, R. J . Electrochem. Soc. 1997, 144, L23.

(251) Tazi, B.; Savadogo, O. Electrochim. Acta 2000, 45, 4329.

(252) Tazi, B.; Savadogo, O.J . New Mater. Electrochem. Systems 2001, 4, 187.

(253) Bonnet, B.; J ones, D. J .; Rozière, J .; Tchicaya, L.; Alberti, G. J New Mater. Electrochem. Syst. 2000, 3, 87.

(254) Weng, D.; Wainright, J. S.; Landau, U.; Savinell, R. F. J. Electrochem. Soc. 1996, 143, 1260.

(255) Edmondson, C. A.; Stallworth, P. E.; Chapman, M. E.; Fontanella, J . J .; Wintersgill, M. C.; Chung, S. H.; Greenbaum, S. G. Solid State I onics 2000, 135, 419.

(256) Edmondson, C. A.; Stallworth, P. E.; Wintersgill, M. C.; Fontanella, J . J .; Dai, Y.; Greenbaum, S. G. Electrochim. Acta 1998 43, 1295

(257) Wintersgill, M. C.; Fontanella, J . J . Electrochim. Acta 1998, 43, 1533

(258) Zawodzinski, T. A.; Neeman, M.; Sillerud, L. O.; Gottesfeld, S. J . Phys. Chem. 1991, 95, 6040.
(259) Edmondson, C. A.; Fontanella, J . J .; Chung, S. H.; Greenbaum, S. G.; Wnek, G. E. Electrochim. Acta 2001, 46, 1623.

(260) Edmondson, C. A.; Fontanella, J. J . Solid Statel onics 2002, 152$153,355$.

(261) Fontanella, J . J .; McLin, M. G.; Wintersgill, M. C.; Calame, J P.: Greenbaum, S. G. Solid State I onics 1993, 66, 1.

(262) Ren, X.; Springer, T. E.; Zawodzinski, T. A.; Gottesfeld, S. J Electrochem. Soc. 2000, 147, 466.

(263) Hietala, S.; Maunu, S. L.; Sundholm, F. J . Polym. Sci., Part B: Polym. Phys. 2000, 38, 3277.

(264) Doyle, M.; Rajendran, G. In Handbook of Fuel Cells-Fundamentals, Technol ogy and Applications; Vielstich, W., Lamm, A., Gasteiger, H. A., Eds.; Wiley: Chichester, U.K., 2003, p 351.

(265) Motupally, S.; Becker, A.J .; Weidner, J . W. J . Electrochem. Soc 2000, 147, 3171.

(266) Verbrugge, M. W. J . Electrochem. Soc. 1989, 136, 417.

(267) LaConti, A. B.; Fragala, A. R.; Boyack, J. R. In Proceedings of theSymposium on Electrode Materials and Processes for Energy Conversion and Storage; Mcl ntyre, J. D. E., Srinivasan, S., Will, F. G., Eds.; The Electrochemical Society Softbound Proceedings Series, PV 77-6; The Electrochemical Society: Princeton, NJ , 1977; p 354.

(268) Meier, F.; Kerres, J .; Eigenberger, G. J . New Mater. Electrochem. Syst. 2002, 5, 91

(269) Helmholtz, H. Weid. Ann. 1879, 7, 337.

(270) Lamb, H. Philos. Mag. 1888, 5, 52

(271) Perrin, J.J . Chim. Phys. 1904, 2, 601

(272) Smoluchowski, M. Handbuch der Elektrizität und des Magne tismus, Vol. II; Barth: Leipzig, 1914.

(273) Breslau, B. R.; Miller, I. F. Ind. Eng. Chem. Fundam. 1971, 10 554.

(274) Ise, M.; Kreuer, K. D.; Maier, J . Solid State Ionics 1999, 125 213.

(275) Okada, T.; Moller-Holst, S.; Gorseth, O.; Kjelstrup, S. J . Electroanal. Chem. 1998, 442, 137.

(276) Ren, X.; Gottesfeld, S. J . Electrochem. Soc. 2001, 148, A87.

(277) Zawodzinski, T. A.; Davey, J .; Valerio, J .; Gottesfeld, S. Electrochim. Acta 1995, 40, 297

(278) Zawodzinski, T. A.; Derouin, C.; Radzinski, S.; Sherman, R. J Smith, Van T.; Springer, T. E.; Gottesfeld, S. J . Electrochem. Soc. 1993, 140, 1041

(279) Ren, X.; Henderson, W.; Gottesfeld, S. J . Electrochem. Soc. 1997 144, L267.

(280) Xie, G.; Okada, T. J . Electrochem. Soc. 1995, 142, 3057.

(281) Fuller, T. F.; Newman, J . J . Electrochem. Soc. 1992, 139, 1332.

(282) Yeo, R. S.; McBreen, J. J. Electrochem. Soc. 1979, 126, 1682.

(283) Ogumi, Z.; Takehara, Z.; Yoshizawa, S. J . Electrochem. Soc. 1984, 131, 769.

(284) Ogumi, Z.; Kuroe, T.; Takehara, Z. J . Electrochem. Soc. 1985 $132,2601$.

(285) Sakai, T.; Takenaka, H.; Wakabayashi, N.; Kawami, Y.; Torikai E. J . Electrochem. Soc. 1985, 132, 1328.

(286) Parthasarathy, A.; Martin, C. R.; Srinivasan, S. J . Electrochem Soc. 1992, 138, 916.

(287) Broka, K.; Ekdunge, P. J . Appl. Electrochem. 1997, 27, 117.

(288) Hietala, S.; Skou, E.; Sundholm, F. Polymer 1999, 40, 5567.

(289) Basura, V. I.; Chuy, C.; Beattie, P. D.; Holdcroft, S. J . Electroanal. Chem. 2001, 501, 77.

(290) Savinell, R.; Yeager, E.; Tryk, D.; Landau, U.; Wainright, J .; Weng, D.; Lux, D.; Litt, M.; Rogers, C. J . Electrochem. Soc. 1994 141, L46.

(291) Sakai, T.; Takenaka, H.; Torikai, E. J . Electrochem. Soc. 1986 $133,88$.

(292) Büchi, F. N.; Wakizoe, M.; Srinivasan, S. J . Electrochem. Soc. 1996, $143,927$.

(293) Kreuer, K. D.; Schönherr, E.; Maier, J . Solid State lonics 1994 $70 / 71,278$

(294) Virkar, A. V.; Baek, H.-D. J . Am. Ceram. Soc. 2002, 85, 3059

(295) Kreuer, K. D.; Maier, J. J . Am. Ceram. Soc. 2004, 87, 1173.

(296) Bonanos, N. Solid State Ionics 1992, 53-56, 967

(297) He, T.; Kreuer, K. D.; Maier, J . Solid Statel onics 1997, 95, 301.

(298) He, T.; Kreuer, K. D.; Baikov, Y. M.; Maier, J . Proc. Electrochem Soc. 1997, 97, 1057.

(299) Gode, P.; Lindbergh, G.; Sundholm, G. J . Electroanal. Chem. 2002, 518, 115

(300) Zang, L.; Ma, C.; Mukerjee, S. Electrochim. Acta 2003, 48, 1845

(301) Tang, H.; Pintauro, P. N. J. Appl. Polym. Sci. 2001, 79, 49.

(302) Wycisk, R.; Pintauro, P. N. J . Membr. Sci. 1996, 119, 155.

(303) Carter, R.; Evilia, R. F.; Pintauro, P. N. J . Phys. Chem. B 2001, 105, 2351

(304) Young, S. K.; Mauritz, K. A. J . Polym. Sci., Part B: Polym. Phys. 2002, 40, 2237.

(305) Dimitrova, P.; Friedrich, K. A.; Stimming, U.; Vogt, B. Solid State I onics 2002, 150, 115.

(306) Antonucci, P. L.; Arico, A. S.; Creti, P.; Ramunni, E.; Antonucci, V. Solid State I onics 1999, 125, 431.

(307) Kerres, J. J . Membr. Sci. 2001, 185, 3.

(308) Kerres, J .; Ullrich, A.; Hein, M. J . Polym. Sci., Part A: Polym Chem. 2001, 39, 2874. 
(309) Kerres, J.; Van Zyl, A. J . J . Appl. Polym. Sci. 1999, 74, 428.

(310) Kerres, J .; Zhang, W.; Cui, W. J . Polym. Sci., Part A: Polym. Chem. 1998, 36, 1441.

(311) Kerres, J.; Cui, W.; Reichle, S. J . Polym. Sci., Part A: Polym. Chem. 1996, 34, 2421.

(312) Feichtinger, J .; Kerres, J .; Schulz, A.; Walker, M.; Schumacher, J. J . New Mater. Electrochem. Syst. 2002, 5, 155.

(313) Kerres, J .; Zhang, W.; J örissen, L.; Gogel, V. J . New Mater. Electrochem. Syst. 2002, 5, 97

(314) Kerres, J.; Ullrich, A.; Meier, F.; Häring, T. Solid State Ionics 1999, 125, 243.

(315) Kerres, J.; Ullrich, A.; Hein, M.; Gogel, V.; Friedrich, K. A.; Jörissen, L. Fuel Cells 2004, 4, 105

(316) He, R.; Li, Q.; Xiao, G.; Bjerrum, N. J . J . Membr. Sci. 2003, 226 169.

(317) Ma, Y.-L.; Wainright, J. S.; Litt, M. H.; Savinell, R. F. J . Electrochem. Soc. 2004, 151, A8.

(318) Lassègues, J . C.; Grondin, J .; Hernandez, M.; Marée, B. Solid State lonics 2001, 145, 37.

(319) Scharfenberger, G. Ph.D. Thesis, University Mainz, Germany, 2003.

(320) Noda, A.; Susan, A. B.; Kudo, K.; Mitsushima, S.; Hayamizu K.; Watanabe, M. J. Phys. Chem. B 2003, 107, 4024.

(321) Guo, Q.; Pintauro, P. N.; Tang, H.; O'Connor, S. J . Membr. Sci. 1999, 154, 175.

(322) Yang, Y.; Shi, Z.; Holdcroft, S. Macromolecules 2004, 37, 1678.

(323) J ones, D. Presented at EUROMAT 2003, Lausanne, Switzerland

(324) Schuster, M.; Rager, T.; Noda, A.; Kreuer, K. D.; Maier, J . Fue Cells 2004, in press. (Invited paper.)

(325) Campbell, A. N.; Kartzmark, E. M.; Bisset, D.; Bednas, M. E. Can. J . Chem. 1953, 31, 303.

(326) Miyatake, K.; I yotani, H.; Yamamoto, K.; Tsuchida, E. Macromolecules 1996, 29, 6969.

(327) Yang, Y.; Holdcroft, S. Fuel Cells 2004, in press.
(328) Cornet, N.; Diat, O.; Gebel, G.; J ousse, F.; Marsacq, D.; Mercier, R.; Pineri, M. J. New Mater. Electrochem. Syst. 2000, 3, 33.

(329) Genies, C.; Mercier, R.; Sillion, B.; Petiaud, R.; Cornet, N.; Gebel, G.; Pineri, M. Polymer 2001, 42, 5097.

(330) Blachot, J. F.; Diat, O.; Putaux, J.-L.; Rollet, A.-L.; Rubatat, L.; Vallois, C.; Müller, M.; Gebel, G. J. Membr. Sci. 2003, 214, 31.

(331) Piroux, F.; Espuche, E.; Mercier, R.; Pineri, M. J . Membr. Sci. 2003, 223, 127

(332) Harrison, W. L.; Wang, F.; Mecham, J . B.; Bhanu, V. A.; Hill, M.; Kim, Y. S.; McGrath, J. E. J . Polym. Sci., Part A: Polym. Chem. 2003, 41, 2264.

(333) Miyatake, K.; Zhou, H. Y.; Watanabe, M. Macromol ecules 2004 37, 4956.

(334) Susan, M. A. B. H.; Noda, A.; Mitsushida, S.; Watanabe, M. Chem. Commun. 2003, 8, 938

(335) Honma, I.; Nakajima, H.; Nishikawa, O.; Sugimoto, T.; Nomura, S. Solid State I onics 2003, 162-163, 2003.

(336) Honma, I.; Nakajima, H.; Nishikawa, O.; Sugimoto, T.; N omura, S. J. Electrochem. Soc. A 2003, 150, A616.

(337) Kreuer, K. D.; Hampele, M.; Dolde, K.; Rabenau, A. Solid State I onics 1988, 28-30, 589.

(338) Alberti, G.; Costantino, U.; Casciola, M.; Ferroni, S.; Massinelli, L.; Staiti, P. Solid State Ionics 2001, 145, 249.

(339) Baranov, A. I.; Shuvalov, L. A.; Shchagina, N. M. J ETP Lett. 1982, 36, 459.

(340) Haile, S. M.; Boysen, D. A.; Chisholm, C. R. I.; Merle, S. M. Nature 2001, 410, 910

(341) Boysen, D. A.; Uda, T.; Chisholm, C. R. I.; Haile, S. M. Science 2004, 303, 68 .

(342) Coors, W. G. J . Power Sources 2003, 118, 150.

(343) Kreuer, K. D. ChemPhysChem 2002, 3, 771.

CR020715F 